Marquette University

e-Publications@Marquette

\title{
Analysis of the Detection of Organophosphate Pesticides in Aqueous Solutions Using Polymer- Coated SH-SAW Sensor Arrays
}

Tian Newman

Marquette University

\section{Recommended Citation}

Newman, Tian, "Analysis of the Detection of Organophosphate Pesticides in Aqueous Solutions Using Polymer-Coated SH-SAW Sensor Arrays" (2012). Master's Theses (2009 -). Paper 170.

http://epublications.marquette.edu/theses_open/170 


\section{ANALYSIS OF THE DETECTION OF ORGANOPHOSPHATE PESTICIDES IN AQUEOUS SOLUTIONS USING POLYMER-COATED \\ SH-SAW SENSOR ARRAYS}

by

Tian Newman, B.S.

A Thesis submitted to the Faculty of the Graduate School, Marquette University, in Partial Fulfillment of the Requirements for the Degree of Master of Science

Milwaukee, Wisconsin

December 2012 


\section{ABSTRACT \\ ANALYSIS OF THE DETECTION OF ORGANOPHOSPHATE PESTICIDES \\ IN AQUEOUS SOLUTIONS USING POLYMER-COATED \\ SH-SAW SENSOR ARRAYS}

Tian Newman, B.S.

Marquette University, 2012

The shear horizontal surface acoustic wave (SH-SAW) device was investigated as a micro-chemical sensor for the direct, rapid and in-situ monitoring of chemical contaminants in groundwater and wastewater. The chemical contaminants of interest are organo-phosphate-based compounds (parathion, parathion-methyl, and paraoxon). The polymers used as partially chemically selective coatings are 2,2'-diallylbisphenol A 1,1,3,3,5,5-hexamethyltrisiloxane (BPA-HMTS), and 2,2'-diallylbisphenol Apolydimethylsiloxane (BPA-PDMS), polyepichlorohydrin $(\mathrm{PECH})$.

The experimental results indicate that the analyte/polymer interaction is a result of mass loading and viscoelastic changes in the polymer. BPA-HMTS, BPA-PDMS, and $\mathrm{PECH}$ have the highest to lowest sensitivity towards parathion, parathion-methyl, and paraoxon respectively. The polymers tested at a thickness of $0.5 \mu \mathrm{m}$ show a higher (up to $20 \mathrm{kHz}$ ) response than the $0.25 \mu \mathrm{m}$-thick polymers due to a more pronounced effect of mass loading and modulus change. The response times of the polymers from fast to slow are PECH, BPA-PDMS, and BPA-HMTS, and the order for the analytes is paraoxon, parathion-methyl, and parathion respectively.

An array of sensors consisting of devices with different coatings and thicknesses is designed to increase sensor selectivity, which provides a specific pattern for each analyte. Based on visual pattern recognition, analyte identification is performed, using radial plots with the frequency shift and response times of BPA-HMTS, BPA-PDMS, and $\mathrm{PECH}$ at a thickness of $0.5 \mu \mathrm{m}$ as input parameters. The concentration of the unknown sample is determined using an algorithm that computes the unknown sample's distance from the points of the known pattern in the three-dimensional space of the steady-state frequency shifts of the three $0.5 \mu \mathrm{m}$-thick polymer coatings. The probability of the unknown pattern being identical to the known pattern can be determined by a level of confidence.

The sensor is tested for stability, sensitivity, and reproducibility via subjection to a real life application simulation. The sensor is exposed to different interferents such as $\mathrm{pH}$ levels of 6.2, 7.0, and 8.0 and red clay. The sensor response to the analytes is shown to be unaffected by the presence of these interferents. 


\title{
ACKNOWLEDGEMENTS
}

\author{
Tian Newman, B.S.
}

I would like to thank my parents for supporting me throughout my entire academic career, especially my time at Marquette University. Without your encouraging words, I would not have been able to make it this far.

I would also like to thank Dr. Fabien Josse for the time he has invested in my education and encouraging me to move forward and complete my Master's thesis. I would also like to thank Dr. Susan Schneider and Dr. Chung-Hoon Lee for their advice and insight during our seminars. Lastly, I would like to thank Dr. Florian Bender, Dr. Arnold Mensah-Brown, and Dr. Allen Chaparadza for training me and supervising me during my time spent in the Microsensor Research Lab and Chemistry Lab. 
TABLE OF CONTENTS

ACKNOWLEDGEMENTS i

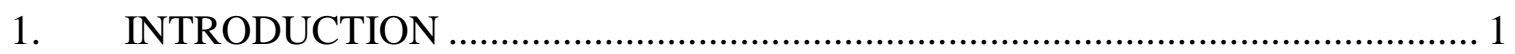

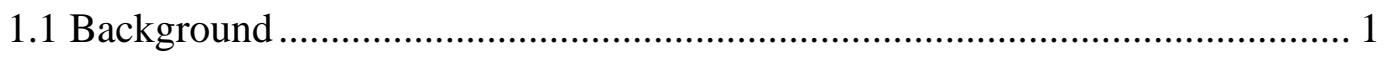

1.2 Overview of Chemical Sensors ................................................................. 2

1.3 Acoustic Wave Devices ....................................................................... 4

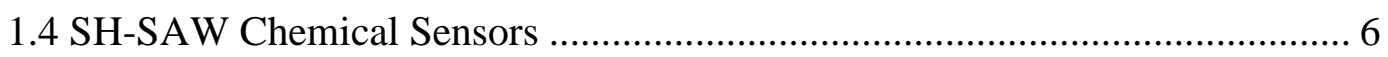

1.5 SH-SAW Sensors and its Uses in Microsensor Arrays ...................................... 7

1.6 Problem Statement and Objective of Research............................................... 8

2. SH-SAW DEVICE USED AS A LIQUID-PHASE SENSOR PLATFORM........ 10

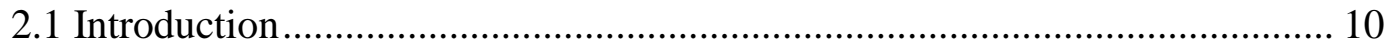

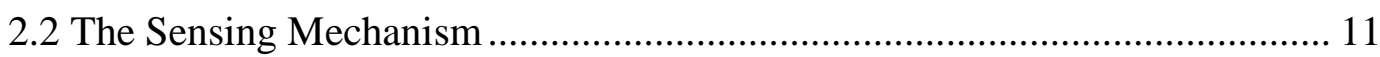

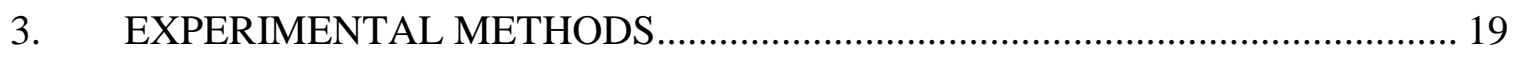

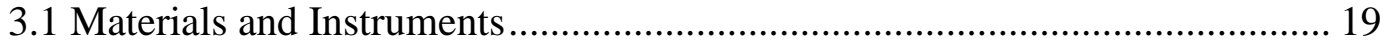

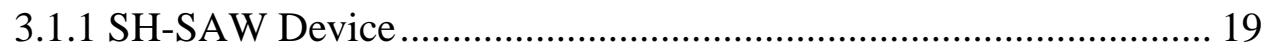

3.1.2 Flow Cell.................................................................................. 20

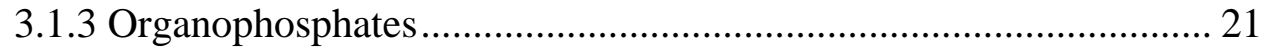

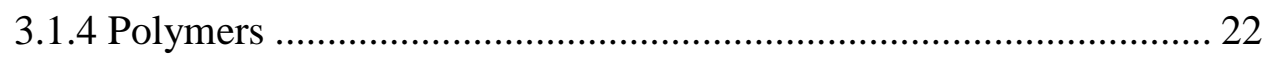

3.1.5 FTIR Spectrometer................................................................... 23

3.1.6 Spin Coater.............................................................................. 23

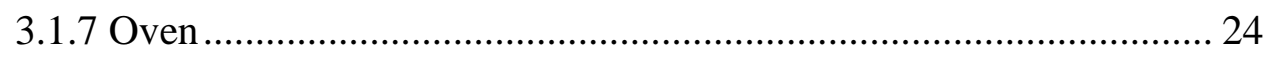

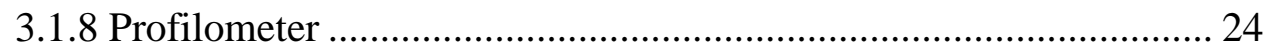

3.1.9 Vector Network Analyzer ............................................................... 25

3.2 Experimental Setup and Procedures …………………............................ 25

3.2.1 Experimental Setup ……………………………….................... 25

3.2.2 SH-SAW Preparation.................................................................. 26

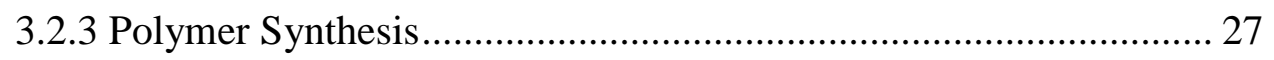

3.2.4 Phosphate Buffer Solution ........................................................... 30

3.2.5 Concentrated Analyte Solution ...................................................... 31

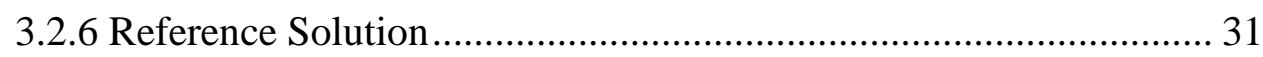

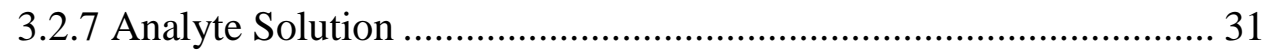




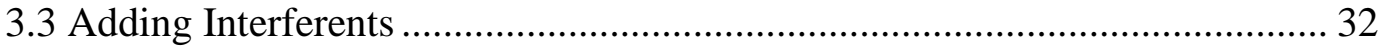

4. RESULTS AND SENSOR SIGNAL ANALYSIS ……………………................... 33

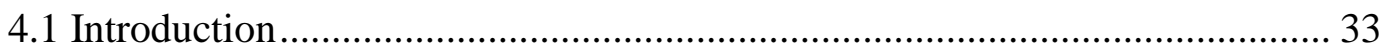

4.2 Sensor Response and Discussion............................................................... 34

4.3 Polymer Viscoelasticity Effect ……………………................................. 51

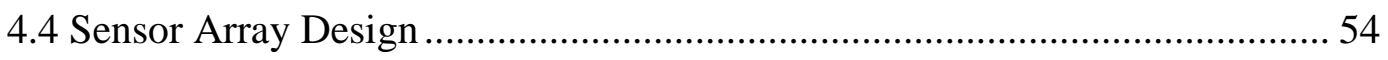

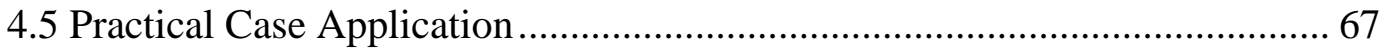

5. SUMMARY, CONCLUSIONS, AND FUTURE WORK ………………………....... 69

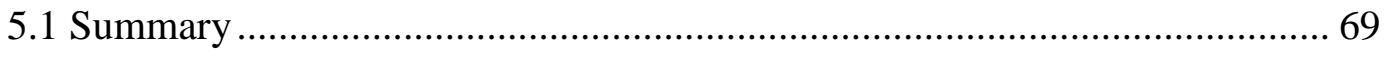

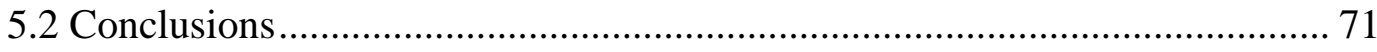

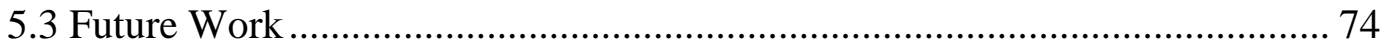

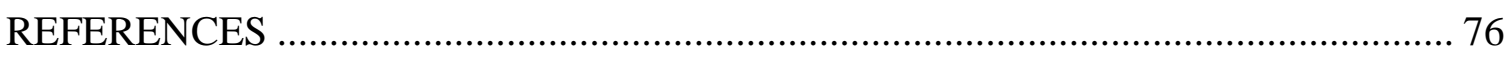

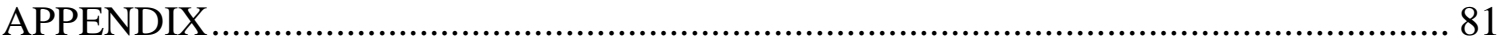




\section{INTRODUCTION}

\subsection{Background}

There are almost 900 different pesticides which are used in the United States. Around 37 of those belong to a class called organophosphates. The chemicals that are contained in organophosphates possess the ability to kill insects by being able to interrupt their brains and nervous systems. Organophosphates are esters of phosphoric acid and prevent an enzyme in the nervous system called cholinesterase from working, concluding in fatal results [1].

Not only are organophosphates a danger to insects but also toxic to human beings and animals. Due to their solubility in water, they can be easily absorbed into the body through ingestion, inhalation, or dermal contact. After their potential as nerve agents had been discovered [2], they were developed and used as neurotoxins in the Second World War. Used as an "agent of opportunity", the military could bomb any vehicle used for transportation of these chemicals resulting in their release into the air [1].

Although different classes of organophosphates have different toxicity levels, the effects will differ depending on the length and the amount of exposure. Resulting symptoms includes headaches, convulsions, coma, etc. [3]. They have been found to be the cause of the symptoms of $4 \%$ of the patients who are treated for poison control due to exposure of pesticides [4]. Due to the harmful effects of these organophosphates, the Environmental Protection Agency (EPA) had made an agreement with the pesticide industry to discontinue its use indoors and it continues to monitor the output in industrial and agricultural settings. 
Today, organophosphates are used as pesticides in a minor scale in the United States which is defined by the EPA as any agricultural land that is less than 300,000 acres [5]. The run-off water from these pesticide-treated agricultural lands will contain small traces of the chemical. In order to test the water for organophosphates, a sample is taken from the contaminated source and brought to a laboratory for analysis. The sample must closely contain the appropriate amount of chemicals present or else even the most hightech machines cannot give a reliable result [6]. During transportation, the sample must be stored at lower temperatures in darkness. The samples will undergo analyte-isolation and enrichment methods [6]. Final determination methods are performed which will help measure lower sample concentrations. While this procedure will often give accurate results, it requires significant manpower and is costly and time consuming. Therefore, there is a need for a portable and low-cost sensor system that can be taken to and installed on sites for real-time monitoring. In addition, sensors have the potential of integration into a sensor array and network to provide selectivity in analyte detection.

\subsection{Overview of Chemical Sensors}

A sensor is a device that receives a stimulus or measurand and converts it into an electrical signal that is compatible with the data acquisition system's interface, i.e. voltage or current [7]. There are several interactions that the measurand can undergo, for example: biological, chemical, or physical. The stimulus can be a chemical compound, a change in velocity, the presence of gas, etc.

The sensor can either be categorized as a passive or an active sensor [7]. An active sensor is one that requires an external signal for operation while a passive device does not. The acoustic wave device is a perfect example of an active device. It requires a 
voltage source to excite the acoustic wave. Another category for a sensor is determined by its measurement strategy, direct or indirect. In a direct sensor, the chemical reaction can produce or affect the measured electrical output. An indirect sensor relies on a secondary source to read the sensed stimulus. These types of sensors usually need a transducer which has the capability to convert a signal from one form to another.

More specifically, chemical sensors can be described as sensors in which an interaction between an analyte and a sensitive coating occurs. This interaction can be divided into two main categories: physical or chemical. When it comes to the context of adsorption, chemical sensors employ the chemisorption process which is typically a high energy process and is sometimes irreversible.

Important parameters of chemical sensors include sensitivity (which is related to detection limit and resolution), selectivity, and reproducibility. The detection limit of a sensor is the smallest concentration of the measurand that can be detected, and its resolution is its accuracy in quantifying this concentration. This can be important in evaluating the performance of a sensor. A chemical sensor is described to be selective if it only responds to the measurand of interest. Often, a coating or receptor has only partial selectivity to an analyte, meaning that it shows a strong response to the measurand of interest but also responds to other analytes, albeit with a lower sensitivity in most cases. Selectivity for a measurand can be increased when there are several sensors placed in an array, each showing a degree of partial selectivity for the analyte of interest.

Chemical sensors can be based on different sensing mechanisms, including electrochemical sensors, optical sensors, acoustic-wave sensors, and chemiresistive sensors [8]. Electrochemical sensors use a redox reaction to measure analyte 
concentrations in liquid [9]. Electrochemical sensors are more commonly used for liquid sensing but are also used in gas sensing. Optical sensors can use intrinsic absorption or fluorescence to give a visual representation (through a change in color) of the contamination amount [8]. Chemiresistive sensors measure the change in electrical resistance of a coating which interacts with the analyte. The chemical sensor of interest in this work will be a mass and viscoelasticity-sensitive sensor, based on acoustic waves, which utilizes sensitive coatings as the interface between device and medium to be analyzed.

\subsection{Acoustic Wave Devices}

The first large scale commercial application of surface acoustic wave devices were filters for cell phones but it has found its ways into automotive, medical, industrial and commercial applications [10]. There are several different types of acoustic wave devices. The first acoustic wave device fabricated was the Quartz Crystal Microbalance (QCM) which was initially used to measure film thickness by measuring added mass to the surface. By making the film a sorptive chemical coating, it could be used as a chemical sensor [11]. The technique to perform chemical sensing was then improved when the surface-acoustic-wave delay line using interdigital transducers was invented [12]. The acoustic-plate-mode delay line and flexural-plate-wave device are both devices that use the same fundamental principles of wave generation and detection but different modes of elastic wave propagation [13].

In the present work, the shear-horizontal surface acoustic wave device is used. This surface acoustic wave device uses two sets of interdigital transducers which are fabricated onto a piezoelectric crystal using a photolithographic process. The purpose of 
the first transducer is to convert an electrical signal into a mechanical wave using the piezoelectric effect and the second is to reverse the process. The generated acoustic wave interacts with surface perturbations on the transducers and/or on the propagation path. There are several types of piezoelectric crystals that can be used for the acoustic wave sensor device. The most popular substrate materials are quartz $\left(\alpha-\mathrm{SiO}_{2}\right)$, lithium tantalate $\left(\mathrm{LiTaO}_{3}\right)$, and lithium niobate $\left(\mathrm{LiNbO}_{3}\right)$. Each material has its own properties that make it the preferred choice for a given application. The properties include cost, temperature dependence of the acoustic wave velocity, acoustic attenuation, permittivity, and propagation velocity [10]. The decision for which substrate is the most appropriate depends on the application of the sensor.

There are different modes of propagation for each acoustic wave device, depending on the crystal type, cut and orientation [10]. They are categorized by their velocity and displacement directions. The SAW mode of propagation was discovered by Lord Rayleigh and therefore named Rayleigh waves [14]. Rayleigh waves can be described as longitudinal which means the particle displacement is in the same direction as the wave propagation and shear which means the particle displacement is perpendicular to the direction of propagation of the wave, such that a given atom at the surface follows an elliptical path. The energy couples with any layer that is adhered to the surface of the substrate which affects the magnitude and velocity of the wave. In return, the device is able to detect changes in mass and changes in mechanical properties. The acoustic energy that comes from a Rayleigh wave is confined within one wavelength of the surface [10]. This allows for high sensitivity to surface perturbations but because of its shear vertical component, the Rayleigh wave can couple to compressional waves in 
adjacent liquid, causing excessive attenuation of the wave. For this reason, this type of wave is not used in liquid sensing applications. Some piezoelectric crystal material can be polarized allowing the particle displacement to be parallel to the sensing surface (shear horizontal) or for the particle displacement to be perpendicular to the surface (shear vertical). An example of this type of cut can result in a shear horizontal acoustic plate mode. They are not very sensitive to surface perturbations because the energy of the wave is confined between the surfaces of the substrate [15]. The shear horizontal surface acoustic wave mode is preferable for liquid phase sensing because it minimizes coupling to compressional waves in the liquid which would result in acoustic loss.

The sensitivity of an acoustic wave sensor is relative to the amount of energy that is being perturbed [10]. Waves that travel on the surface of the device are called surface waves with examples including the Rayleigh SAW and the surface transverse wave (STW) devices. The waves that travel through the substrate are called bulk waves with the resulting devices known as bulk acoustic wave (BAW) devices and include examples such as the shear-horizontal acoustic plate mode (SH-APM) and thickness-shear mode (TSM). The best mode in the case of liquid sensing is the shear-horizontal surface acoustic wave (SH-SAW) which permits particle displacement in the shear horizontal direction instead of the shear vertical direction. This would result in a higher energy density at the surface of the device. [10].

\subsection{SH-SAW Chemical Sensors}

As stated previously, shear-horizontal surface acoustic wave devices are created when the cut of the piezoelectric crystal is rotated so the mode changes from shear vertical to shear horizontal. This will produce a particle displacement parallel to the 
device surface. The SH-SAW device configuration is explained in greater detail in Chapter 2.

The range for uses of SH-SAW devices can be expanded when a sensitive coating is applied to the top surface of the substrate. These types of devices can be classified as SH-SAW chemical sensors. The sensitive coating's properties can change such as mass, elasticity and/or conductivity when exposed to a chemical stimulus [10]. The coating acts as a waveguide and traps the acoustic energy to the surface of the substrate. This lowers the radiation losses and increases sensitivity to surface perturbations when liquids are introduced to the system, giving it the ability to act as a liquid phase sensor.

There are several design features that need to be taken into consideration when choosing a chemically sensitive coating. The coating-analyte binding needs to be reversible meaning the polymer needs to be capable of absorbing analyte and then releasing all of it when flushed out with a neutral solution. The coating should also possess a fairly quick response time when the analyte is being absorbed and desorbed. The coating also needs to be stable and robust over a long time period; it should not be easily removed by the passing of liquid. The coating needs to be very selective to the analyte of interest. It is also very important for it to be reproducible and sensitive. The sensitivity and selectivity of a chemical sensor can be improved by utilizing selective polymer coatings in a sensor array.

\subsection{SH-SAW Sensors and its Uses in Microsensor Arrays}

A sensor array has the ability to eliminate any selectivity problems if there is a multicomponent sample or if any interferents are present. This can be done by utilizing the selective molecular interaction during the chemisorption process. Several identical 
SAW sensor platforms will be coated with partially selective sensitive coatings and placed in an array. The SAW sensors will be placed in a row allowing each one to be exposed to the same analyte solution. Each sensor will produce a different and unique response.

The two most important aspects to designing an effective sensor array is ensuring the appropriate selection of sensitive coatings for each element in the array and accurately identifying the chemical of interest through data collection and pattern recognition software. Previous work focuses on several types of coating selections for a sensor array [16]. These methods include the Cluster and K-Means Analysis which group dissimilar/similar coating responses respectively into clusters. The coatings are interchangeable depending on their contribution to the performance of the array [16]. The parameters observed in this work will be the steady state response and the response time for each coating/analyte combination.

\subsection{Problem Statement and Objective of Research}

Currently, there is no portable or low cost chemical sensor which exists for detection of organophosphates in aqueous solutions. A commonly used procedure consists of sampling the liquid from the source and transporting it to a laboratory for analysis, a process which is both time-consuming and labor-intensive [6].

The goal of this project is to investigate and design micro-chemical sensors for the direct, rapid and in-situ monitoring of chemical contaminants in groundwater and wastewater. The chemical contaminants of interest include organic compounds and organo-phosphate-based compounds (pesticides, etc.). The guided shear horizontal surface acoustic wave (SH-SAW) device will be used for this application. The device will 
use chemically modified surfaces, which show stability in water, to achieve low detection limits with class specificity. Chemically sensitive coatings will be studied and characterized for optimal performance, (i.e., sensitivity, reproducibility and electrical passivation). After determining sensor ruggedness, the devices will be configured and optimized for the analytes of interest (phosphorus-based compounds: parathion, paraoxon, and parathion-methyl) and individual sensors will be fabricated and characterized.

Furthermore, multiple sensors are required when monitoring samples which contain several chemicals. In these cases, it would be more beneficial to have one device that contains multiple delay lines each with a different selective coating. Another objective of this research is to design a sensor array that will identify the organophosphates and quantify their concentrations. Polymers in the array will include polyepichlorohydrin (PECH), 2,2'-diallylbisphenol A - 1,1,3,3,5,5-hexamethyltrisiloxane (BPA-HMTS), and 2,2'-diallylbisphenol A - 1,1,3,3,5,5-polydimethylsiloxane (BPAPDMS). The latter (BPA-HMTS and BPA-PDMS) were designed and synthesized in the Microsensor Research Laboratory. 


\section{SH-SAW DEVICE USED AS A LIQUID-PHASE SENSOR PLATFORM}

\subsection{Introduction}

The SH-SAW device has been proven to be a good candidate for sensing in biological and chemical applications in the liquid phase [10]. As previously discussed in Chapter 1, the SH-SAW mode of propagation has a particle displacement that takes place predominantly in the shear horizontal direction $\left(\mathrm{u}_{2}\right)$, parallel to the surface of the device. The $\mathrm{SH}$ displacement is associated with the lowest loss in the liquid phase. The $\mathrm{SH}-$ SAWs also propagate deeper within the substrate making it less sensitive to surface perturbations. To improve device sensitivity, a thin wave-guiding layer is added to the surface of the device. This will trap the acoustic energy near the surface and increase sensitivity to perturbations along the wave propagation path $[17,18,19]$.

The SH-SAW sensor configuration used in this work is the three layer geometry. The layers include the liquid, polymer, and substrate.

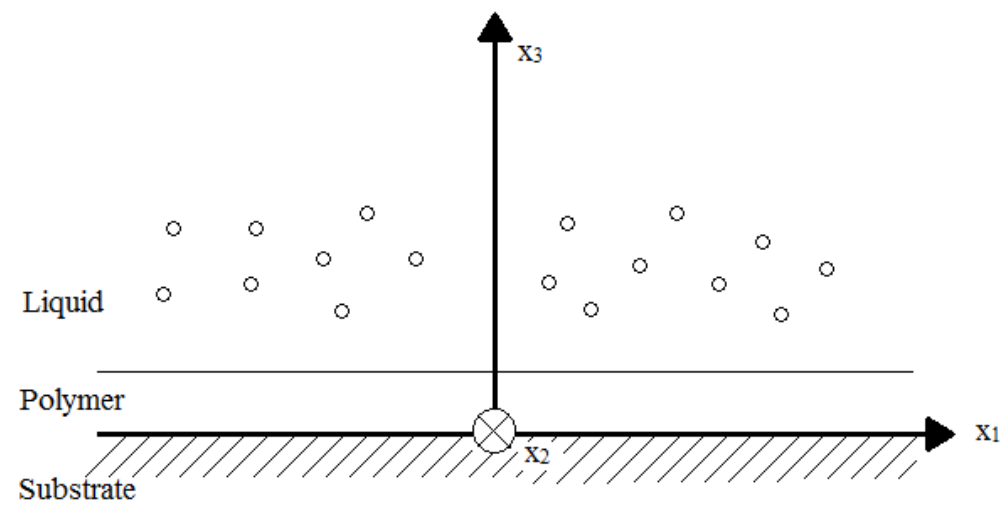

Figure 2.1: Three layer acoustic wave sensor geometry 
The liquid layer is assumed to be a Newtonian fluid (exemplifying fluidic properties no matter the forces acting upon it) because the solutions undergoing testing are very dilute aqueous solutions. The polymer layer (the waveguiding layer) has a finite thickness and is assumed to have a slower shear wave velocity than the substrate, which is a precondition for confining the wave to the surface [20]. It is also used as the sensing layer. The substrate is made from a piezoelectric crystal. The liquid layer and substrate are considered to be semi-infinite layers. The most commonly used substrate materials are Quartz $\left(\alpha-\mathrm{SiO}_{2}\right)$, Lithium Tantalate $\left(\mathrm{LiTaO}_{3}\right)$, and Lithium Niobate $\left(\mathrm{LiNbO}_{3}\right)$ [10]. When considering the optimal substrate for sensing applications, the most important properties that are taken into consideration are high electromechanical coupling coefficient and low temperature coefficient. The substrate with the best compromise between the two is the $36^{\circ}$ Rotated $\mathrm{Y}$-cut X-propagating $\mathrm{LiTaO}_{3}$.

\subsection{The Sensing Mechanism}

In the case of the SH-SAW device, the acoustic wave is propagating horizontally across the device and the particle displacement is perpendicular to the direction of propagation. This wave can be expressed by [21]

$$
u\left(x_{1}, x_{2}, x_{3}, t\right)=u\left(x_{2}, t\right) e^{j \omega t-\gamma x_{1}}
$$

where $\omega$ is the angular frequency $(2 \pi f)$ and $\gamma$ is a complex propagation factor representing attenuation $\alpha$ and wavenumber $k$, given by [21]

$$
\gamma=\alpha+j k=\alpha+j \frac{\omega}{v}
$$

If the frequency is constant, changes in wave propagation can be represented by

$$
\Delta \gamma=\Delta \alpha-j k_{0} \frac{\Delta v}{v_{0}}
$$


where $k_{0}$ is the unperturbed wavenumber and $v_{0}$ is the unperturbed acoustic wave phase velocity. Eq. 2.3 can be represented in its normalized form

$$
\Delta \gamma^{\prime}=\frac{\Delta \gamma}{k_{0}}=\frac{\Delta \alpha}{k_{0}}-j \frac{\Delta v}{v_{0}}
$$

When SAW devices are used as sensors, the measured responses occur from perturbations in wave propagation characteristics (wave velocity and attenuation) [17, 22]. This is due to the interaction between the SAW and the probed medium. [21] Because the substrate is a piezoelectric crystal and the film is in direct contact with (and rigidly bonded to) the substrate, mechanical and electrical coupling will exist between the SAW and the polymer layer. There are several types of interactions that take place between the SAW and the polymer layer to cause changes in the wave velocity and attenuation [23]. The interactions that are due to mechanical coupling are mass loading and elastic and viscoelastic effects. The interactions that are due to electric coupling are acoustoelectric interaction. Therefore the wave can be analyzed from the fractional change in phase velocity $\left(\frac{\Delta v}{v_{0}}\right)$ and normalized attenuation $\left(\frac{\Delta \alpha}{k_{0}}\right)$ in eq. 2.4 [24].

The changes in phase velocity and attenuation are all dependent on the following variables: elastic constant $\mathrm{c}$, dielectric constant $\varepsilon$, conductivity $\sigma$, temperature $\mathrm{T}$, and pressure $\mathrm{P}$. The change in mass loading, $\Delta \mathrm{m}$, will only affect the phase velocity. The expression can be written as a partial derivative with respect to each variable [25]

$$
\begin{gathered}
\Delta v=\left(\frac{\partial v}{\partial \mathrm{m}}\right) \Delta \mathrm{m}+\left(\frac{\partial v}{\partial \mathrm{c}}\right) \Delta \mathrm{c}+\left(\frac{\partial v}{\partial \varepsilon}\right) \Delta \varepsilon+\left(\frac{\partial v}{\partial \sigma}\right) \Delta \sigma+\left(\frac{\partial v}{\partial \mathrm{T}}\right) \Delta \mathrm{T}+\left(\frac{\partial v}{\partial \mathrm{P}}\right) \Delta \mathrm{P}, \\
\Delta \alpha=\left(\frac{\partial \alpha}{\partial \mathrm{c}}\right) \Delta \mathrm{c}+\left(\frac{\partial \alpha}{\partial \varepsilon}\right) \Delta \varepsilon+\left(\frac{\partial \alpha}{\partial \sigma}\right) \Delta \sigma+\left(\frac{\partial \alpha}{\partial \mathrm{T}}\right) \Delta \mathrm{T}+\left(\frac{\partial \alpha}{\partial \mathrm{P}}\right) \Delta \mathrm{P}
\end{gathered}
$$

In these equations the terms for the different variables are assumed independent, which will be the case for small perturbations. The dual delay line design of this device 
allows for a sensing line and a reference line. Temperature and pressure effects are eliminated through differential measurements [19]. The phase velocity and attenuation is now a function of mechanical and acoustoelectric interactions. The dielectric constant and conductivity (acoustoelectric interactions) are minimized by the metalized delay path between the IDTs. This results in a device that is purely affected by mechanical interactions and can be represented by eqs. 2.7 and 2.8. As a result, the film undergoes both translation and deformation. Translational motion affects the SAW propagation velocity and is affected by the mass loading response of the film. Film deformation leads to energy storage and power dissipation which are both contributions to SAW velocity and attenuation [26].

$$
\begin{gathered}
\Delta v=\left(\frac{\partial v}{\partial \mathrm{m}}\right) \Delta \mathrm{m}+\left(\frac{\partial v}{\partial \mathrm{c}}\right) \Delta \mathrm{c} \\
\Delta \alpha=\left(\frac{\partial \alpha}{\partial \mathrm{c}}\right) \Delta \mathrm{c}
\end{gathered}
$$

Note that for this work, a sensor design is used where the IDTs are directly immersed in the sample liquid. This is possible due to the high dielectric constant of $\mathrm{LiTaO}_{3}$, which ensures that a sufficient part of the electric field in the IDTs will remain in the sensor substrate as required for efficient piezoelectric transduction. Generally, the sample liquid will also affect the electrical properties of the IDTs, such as capacity. However, since the IDTs are protected with a non-conducting polymer layer, and since only dilute aqueous solutions are used in the experiments, these interactions can be neglected in the analysis.

The viscoelastic properties of a polymer can be represented by its complex modulus. The viscoelasticity of a polymer describes how the polymer deforms in response to an applied stress [21]. It is represented by the shear modulus 


$$
G=G^{\prime}+j G^{\prime \prime},
$$

where $\mathrm{G}^{\prime}$ represents the shear storage modulus related to energy storage and release during the periodic deformation associated with the oscillating stress and G"represents the shear loss modulus related to energy loss usually seen as heat dissipation [21, 22].

Using eqs. 2.7, 2.8 and 2.9, the change in frequency and attenuation can be written as functions of the mass loading and the shear modulus by

$$
\Delta \mathrm{f}=\mathrm{g}_{1}\left(\Delta \mathrm{m}, \Delta \mathrm{G}^{\prime}, \Delta \mathrm{G}^{\prime \prime}\right)
$$

and

$$
\Delta \alpha=g_{2}\left(\Delta G^{\prime}, \Delta G^{\prime \prime}\right)
$$

In this work the frequency is measured over the phase velocity because the fractional change in frequency is proportional to the fractional change in phase velocity (assuming there is no dispersion in the wave). The relationship is shown below. The operational frequency point of the device is given by

$$
\mathrm{f}_{\mathrm{o}}=\frac{v_{\mathrm{o}}}{\mathrm{d}}
$$

where $v_{\mathrm{o}}$ is the phase velocity and $\mathrm{d}$ is the IDT periodicity. The transducers are most efficient when the wavelength matches length $d[22]$.

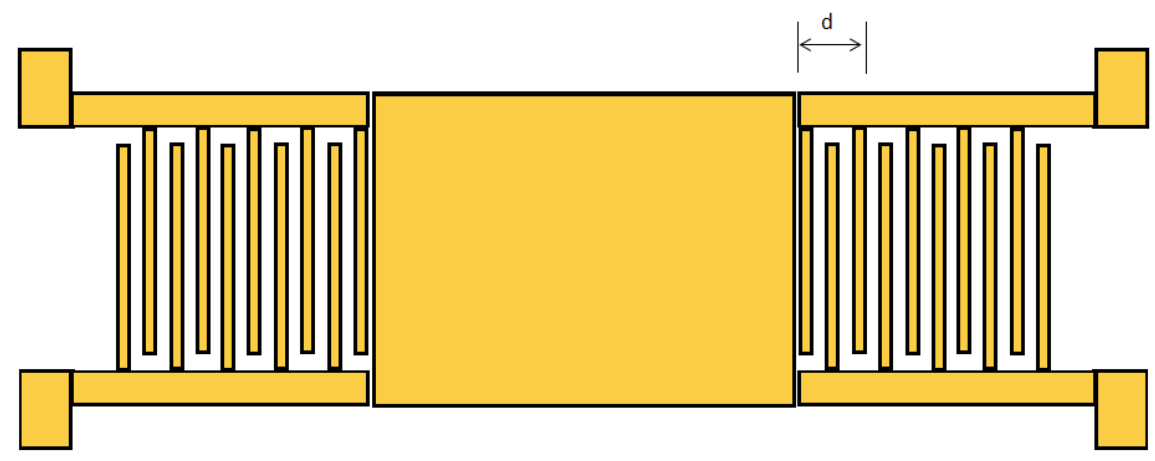

Figure 2.2: Schematic of SH-SAW sensor 
In this work, the metalized delay path and IDTs are both coated with a polymer film and therefore the propagating wave is affected by any perturbations on the surface of the device. Therefore the fractional change in frequency can be given by

$$
\frac{\Delta \mathrm{f}}{\mathrm{f}}=\frac{\Delta v}{v} .
$$

Attenuation measurements are performed using a vector network analyzer (VNA). The VNA measures the insertion loss of the device which can be converted to attenuation using [21]

$$
\frac{\Delta \alpha}{k}=\frac{\Delta L_{1}}{54.6 N_{\lambda}}
$$

where $\Delta L_{1}$ is the change in insertion loss expressed in $\mathrm{dB}$ and $N_{\lambda}$ is the center-to-center IDT spacing expressed in acoustic wavelengths.

\subsection{The Sorption Process}

In order to measure the response of a sensor (phase velocity and attenuation), it is important to know how the polymer reacts when exposed to an analyte. A process called sorption occurs, the penetration and dispersal of analytes into polymer coatings which covers adsorption and absorption [27]. Adsorption occurs when the molecules of the analyte interact with the polymer at the surface of the layer. If these interactions are weak, this process can be called physisorption. Weak bonds between molecules will allow for the sorption process to be reversible (optimal for chemical sensors). When the polymer is introduced to the analyte, the sorption process continues to occur until the concentration of the adsorbing species reaches its saturation value. $K_{C}$ is a partition 
coefficient which describes the ratio of analyte concentration sorbed in the polymer to analyte concentration in the liquid at equilibrium [21, 28]. It can be written as

$$
K_{c}=\frac{C_{s}}{C_{a}}=\frac{\left(m_{s} / V_{s}\right)}{C_{a}}
$$

where $C_{s}$ is the concentration of the analyte sorbed in the polymer, $C_{a}$ is the concentration of the analyte in the aqueous solution, and $m_{s}$ is the mass of the analyte sorbed into the coating with volume $V_{S}[24] . K_{C}$ can be related to the solubility of an analyte in aqueous solution. Solubility defines whether an analyte is more likely to partition into the liquid or coating. When the solubility of an analyte in aqueous solution is low, the analyte is more likely to partition into the polymer coating and therefore $K_{C}$ will be high.

This sorption process can be expressed in a first-order difference equation. This mathematical model is shown below

$$
C_{K+1}=C_{K}+S\left(K_{C} C_{s}-C_{K}\right)
$$

where $C_{K}$ represents the concentration of analyte in the polymer coating at time $t_{K}, S$ is the sorption rate at which the analyte is being sorbed into the coating.

While eq. 2.16 represents the sorption process using discrete data points, the sensor response can also be described using a continuous model given below

$$
\dot{C}(t)=\left(1 / \tau_{s}\right)\left(K_{C} C_{a}(t)-C(t)\right), \quad \tau_{s}>0
$$

where $1 / \tau_{s}$ represents the absorption rate coefficient and $\dot{C}(t)=\frac{d C(t)}{d t}$ [29]. It is assumed that the mass loading changes and modulus change are proportional to the concentration of the solution at small concentrations and the temperature remains constant. Equation 2.17 can be put in a state-space format [29] 


$$
\dot{C}(t)=-\left(1 / \tau_{s}\right) C(t)+\left(1 / \tau_{s}\right)\left(K_{C} C_{a}(t)\right)
$$

When the state-space equation is solved, the frequency change can be modeled using

$$
\Delta f(t)=\Delta f(\text { steady }- \text { state }) *\left(1-e^{\left(-t / \tau_{s}\right)}\right)
$$

For sensing in the gas phase, the frequency response predominantly due to mass loading effects can be expressed by [28, 30]

$$
\Delta f_{v}=\Delta f_{\text {poly }} C_{v} K / \rho_{\text {poly }}
$$

where $\Delta f_{\text {poly }}$ is the frequency shift due to the added polymer layer, $C_{v}$ is concentration of the vapor, $\rho_{\text {poly }}$ is the density of the polymer with the analyte sorbed in the coating with volume $V_{S}$, and $K$ is the polymer-vapor partition coefficient.

Using this as a reference, it is possible to model the frequency response for liquid phase sensing. This must take into account the frequency shift that occurs when the polymer is added to the sensing surface and when the liquid is introduced into the system. In this case the liquid is a mixture of water and methanol. The relationship of $\Delta f_{c}$, the frequency shift due to the coating which has absorbed the water and methanol, and $\Delta f_{a}$, the frequency shift due to analyte, can be shown by [21]

$$
\frac{\Delta f_{a}(\text { mass })}{\Delta f_{c}}=\frac{m_{s}}{m_{\text {poly }}+m_{m}+m_{w}}=\frac{m_{s}}{V_{s}\left(\rho_{\text {poly }}+K_{m} C_{r e f}+C_{w}\right)}
$$

where $m_{\text {poly }}$ is the mass of the polymer, $m_{m}$ is the mass of the absorbed methanol in the polymer, $m_{w}$ is the mass of the sorbed water, $K_{m}$ is the partition coefficient for methanol, $C_{r e f}$ is the concentration of methanol in the reference solution, and $C_{w}$ is the concentration of water in the polymer. Take note that eq. 2.21 describes the frequency change due to mass loading only. Substituting eq. 2.15 into eq. 2.21 , the following equation can be found 


$$
\Delta f_{a}(m a s s)=\Delta f_{C}\left(\frac{C_{s} K_{C}}{\rho_{p o l y}+K_{m} C_{r e f}+C_{w}}\right)
$$

When the analyte is introduced to the polymer, the viscoelastic properties of the polymer are changed. This effect is based on the physicochemical interaction that occurs between the polymer and analyte and the amount of analyte that is absorbed by the polymer. The polymer reacts in two main ways through swelling and plasticization (polymer softening) [21]. During the swelling process the analyte affects the intermolecular forces that are keeping the individual polymer chains together [21]. The amount of swelling is dependent on the strength of the forces. At the same time, the newly absorbed analyte acts as a lubricant which allows the polymer chain to move more freely and result in a softer polymer [21]. This leads to an overall change in $\mathrm{T}_{\mathrm{g}}(\mathrm{a}$ temperature at which the polymer transitions from glassy to elastomeric), with the phase diagram of the polymer showing a larger elastomeric region. While the changes in mass accumulation have very small effects in attenuation, the change in modulus can result in changes in both velocity and attenuation [22]. The total frequency change can be represented by

$$
\Delta f_{a}(\text { total })=\Delta f_{c}\left(\frac{C_{s} K_{C}}{\rho_{p o l y}+K_{m} C_{r e f}+C_{w}}\right)+\Delta f_{a}(\operatorname{modulus})
$$

where $\Delta f_{a}(\operatorname{modulus})=\kappa \Delta f_{c}$ and $\kappa$ is a parameter that includes all material property effects such as the SH-SAW sensor, polymer and analyte solution [24]. For small perturbations, $\kappa$ also depends linearly on the concentration and partition coefficient of the analyte [31]. 


\section{EXPERIMENTAL METHODS}

This chapter will discuss the materials, instruments, and procedures that were used to fabricate the polymer coatings (PECH, BPA-HMTS, and BPA-PDMS) and analyte samples (parathion, paraoxon, and parathion-methyl) as well as how they were characterized. The chapter will also cover the measurement setup and process for the sensor parameters that need to be collected. The parameters include frequency shift, change in loss, and phase shift. This chapter will also discuss the influence of various parameters on the system such as the $\mathrm{pH}$ level and other physical or chemical interferents.

\subsection{Materials and Instruments}

\subsubsection{SH-SAW Device}

The shear horizontal surface acoustic wave (SH-SAW) device is fabricated on a $36^{\circ}$-rotated Y-cut X-propagation Lithium Tantalate $\left(36^{\circ} \mathrm{YX}-\mathrm{LiTaO}_{3}\right)$ crystal with $10 / 80$ $\mathrm{nm}$ thick $\mathrm{Cr} / \mathrm{Au}$ IDTs with a wavelength of $40 \mu \mathrm{m}$, corresponding to an operating frequency of $103 \mathrm{MHz}$. A dual delay line configuration is used which allows for the possibility of one line to be used as a reference and the other line as a sensing line. This will minimize any environmental factors such as temperature and pressure effects on the sensor parameters. The device was also designed with a metallized delay line that is placed between the two IDTs to eliminate acoustoelectric interaction with the load [32, 19]. If the acoustoelectric interaction is present, it can introduce reproducibility issues due to fluctuations in the solution's electrical properties [26]. The IDTs are made of gold 
because of its ability to withstand corrosion from the chemical solutions it is exposed to. A layer of chromium is coated underneath the gold to increase the adhesion of the gold to the surface of the substrate [21].

\subsubsection{Flow Cell}

The devices are placed in a flow cell which allows the sensor surface to be exposed to the liquid samples. The cell was designed by F. Josse from Marquette University and R.W. Cernosek from Sandia National Laboratories [33]. It is noted that this cell has been made available to various laboratories around the world for research purposes, and thus can also be found in published literature. The cell is comprised of three parts. The top piece is made from polycarbonate which is able to sustain aqueous solutions over a long period of time. The middle and base pieces are machined from brass. The cell is fabricated using brass to provide electromagnetic shielding to the device from other electrical devices that can produce electromagnetic interference. This interference can affect the sensor response and therefore should be reduced as much as possible [21]. The base contains a recessed area holding the SH-SAW device in place. The middle piece contains contact pins which provide a connection between the device and coaxial cable connectors when placed over the base. It is properly aligned over the base using two alignment pins and tightened with four screws at each corner of the cell. The top covers the middle section and has an inlet and outlet for liquid flow into and out of the cell. It also contains a gasket which provides a pocket for the chemical liquid to be contained over the sensing area. It is kept in place and tightened with four screws at each corner of the cell. 


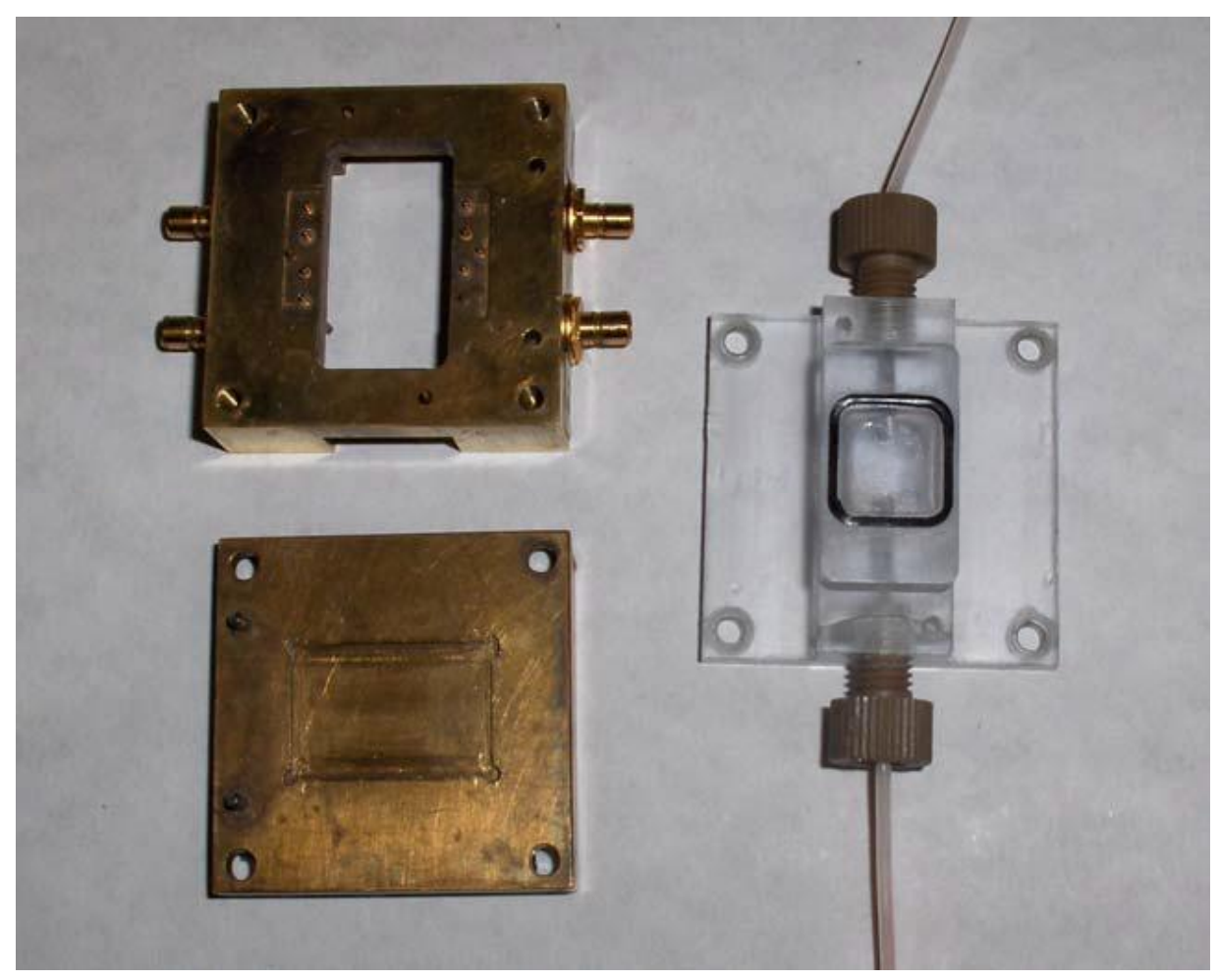

Figure 3.1: The three main pieces of the flow cell: Middle (top left), Base (bottom left), Top (right) [24]

\subsubsection{Organophosphates}

The organophosphates of concern are parathion, paraoxon, and parathion-methyl. They are the basis of many insecticides, herbicides, and nerve gasses. They are highly toxic and dangerous to any type of life form [34].

Parathion and parathion-methyl are mainly used as insecticides on fruits, vegetables, and grains. Because parathion is a carcinogen, exposing the human body to parathion increases the risk of cancer. Its pure form takes the appearance of white crystals but the product bought from Sigma Aldrich comes in liquid form. Parathion's empirical formula is written as follows $\mathrm{C}_{10} \mathrm{H}_{14} \mathrm{NO}_{5} \mathrm{PS}$, with the structure show in Figure 3.2 (a), and has a molecular weight of 291.26 [35, 36]. The empirical formula for parathion-methyl is written as follows $\mathrm{C}_{8} \mathrm{H}_{10} \mathrm{NO}_{5} \mathrm{PS}$, with the structure shown in Figure 3.2 (b), and has a 
molecular weight of 263.21 [37, 36]. Parathion and parathion-methyl need to be stored at a temperature of $2-8^{\circ} \mathrm{C}$. They are insoluble in water and therefore need to be dissolved in a combined aqueous solution of methanol and phosphate buffer solution [35].<smiles>CCOP(=S)(OCC)Oc1ccc([N+](=O)[O-])cc1</smiles>

Figure 3.2 (a): Structure of parathion [35]<smiles>COP(=S)(OC)Oc1ccc([N+](=O)[O-])cc1</smiles>

Figure 3.2 (b): Structure of parathion-methyl [37]

Paraoxon was mainly used as a chemical warfare agent. This product is bought from Sigma Aldrich and comes in liquid form. Its linear formula is $\mathrm{C}_{10} \mathrm{H}_{14} \mathrm{NO}_{6} \mathrm{P}$, with the structure shown in Figure 3.2 (c), and its molecular weight is 275.2 [38]. Paraoxon needs to be stored at a temperature of $25^{\circ} \mathrm{C}$.<smiles>CCOP(=O)(OCC)Oc1ccc([N+](=O)[O-])cc1</smiles>

Figure 3.2 (c): Structure of paraoxon [38]

\subsubsection{Polymers}

The polymers that are used as the sensing layers are 2,2'-diallylbisphenol A 1,1,3,3,5,5-hexamethyltrisiloxane (BPA-HMTS), 2,2'-diallylbisphenol Apolydimethylsiloxane (BPA-PDMS), polyepichlorohydrin $(\mathrm{PECH})$, and poly(methyl methacrylate) (PMMA) [39]. BPA-HMTS and BPA-PDMS are polymers which are 
synthesized at Marquette University using a hydrosilylation reaction of BPA and HMTS, and BPA and PDMS, respectively $[39,40]$. BPA is the organic chemical that provides the functional group for the analyte/coating interaction to take place. The HMTS and PDMS are inorganic chemicals that serve as the porous backbone allowing for rapid analyte absorption.

BPA, HMTS, PDMS, PMMA, and PECH are all purchased from Sigma-Aldrich. PMMA and PECH are diluted using the procedures stated in Section 3.2.3.

\subsubsection{FTIR Spectrometer}

During the fabrication of BPA-HMTS and BPA-PDMS, a Perkin Elmer Spectrum 100 Fourier Transform Infrared (FTIR) spectrometer indicates when the hydrosilylation reaction has taken place by identifying the functional groups of the coating. This FTIR spectrometer can give data collection over a range of 675 to $4000 \mathrm{~cm}^{-1}$ with a resolution of $2 \mathrm{~cm}^{-1}$, taken over 12 scans.

\subsubsection{Spin Coater}

A Specialty Coating Systems (SCS) Model P6024 spin coater was used to evenly distribute a layer of a polymer coating onto the SH-SAW device. This process involves depositing a few drops of polymer solution onto the devices and spinning the device at a high determined spinning rate. The end result is a device with a thin polymer layer on the top surface. The film thickness is dependent on the density, viscosity, and percent weight of the polymer solution and it is also dependent of the spin speed acceleration, ramp time, and hold time [41]. The polymers used for the experiments discussed in this work were spin coated at spin speeds from 2000rpm to 5000rpm, with a ramp time of 3s, and a hold 
time of 30s and 50s. These spin speeds will produce a coating that is approximately $0.25 \mu \mathrm{m}$ and $0.5 \mu \mathrm{m}$ thick.

\subsubsection{Oven}

After spin coating, the PMMA coatings were baked at $180^{\circ} \mathrm{C}$ for two hours in a Lab-Line Imperial V Digital Mechanical Oven Model 3486MB. This treatment ensures that the PMMA forms a stable, glassy uniform polymer layer as required for reference purposes.

\subsubsection{Profilometer}

The Alpha-Step IQ Surface Profiler is an instrument used to measure the thickness of the polymer on a substrate. Due to the polymer film being rubbery at room temperature, a thin film of chromium was deposited over the polymer to prevent the stylus from denting the polymer. Since the profilometer is measuring a step height, the same chromium thickness was deposited on the reference surface (glass substrate) as well. In order to avoid edge effects, the thickness was measured at a distance from the edge of the coating.

\subsubsection{Ellipsometer}

The L2WLSE544 Stokes Ellipsometer [42] is a faster, more efficient, and more accurate way to perform polymer thickness measurements. A layer of chromium is not needed which eliminates the process for metal evaporation. The ellipsometer measures film thickness by shining a polarized laser beam onto a layer and measuring the phase 
shift between the beams reflected from the top and bottom surfaces of the layer. The Stokes ellipsometer uses a two laser system to eliminate ambiguous results [42]. Since the ellipsometer requires no mechanical contact and no coating edge for thickness measurements, the measurement can be performed directly on the sensor device.

\subsubsection{Vector Network Analyzer}

The Agilent 8753ES vector network analyzer was used to characterize the devices and perform sensor measurement by recording various parameters. For device characterization, the network analyzer can make a frequency sweep and display phase and amplitude of the acoustic wave as a function of frequency. For the sensor measurements, the changes in the acoustic wave phase and amplitude (loss) are recorded at constant frequency as a function of time, and in addition the change in frequency is recorded at constant phase as a function of time. It is noted that the measured change in phase could be used to calculate the measured change in frequency.

\subsection{Experimental Setup and Procedures}

\subsubsection{Experimental Setup}

The experimental setup consists of three parts: the flow system (including a peristaltic pump, the flow cell containing the sensor device, PTFE tubing, and vials for sample and waste), a switch/control unit (Agilent 3499A) to alternate between the two delay lines of the sensor device, and the Agilent 8753ES vector network analyzer (VNA). The liquid is brought into the flow cell of the SH-SAW device via the peristaltic pump (Cole Parmer Model 7253-70). The housing cell holds the SH-SAW device and brings 
the analyte in contact with the sensor coating. As previously stated, the flow cell contains integrated pogo pins to provide contact between the IDTs of the sensor device and adapters for coaxial cables. The vector network analyzer sends the measurement data to a computer for storage and analysis. The program used for data collection is based on Agilent VEE 9.2 software.

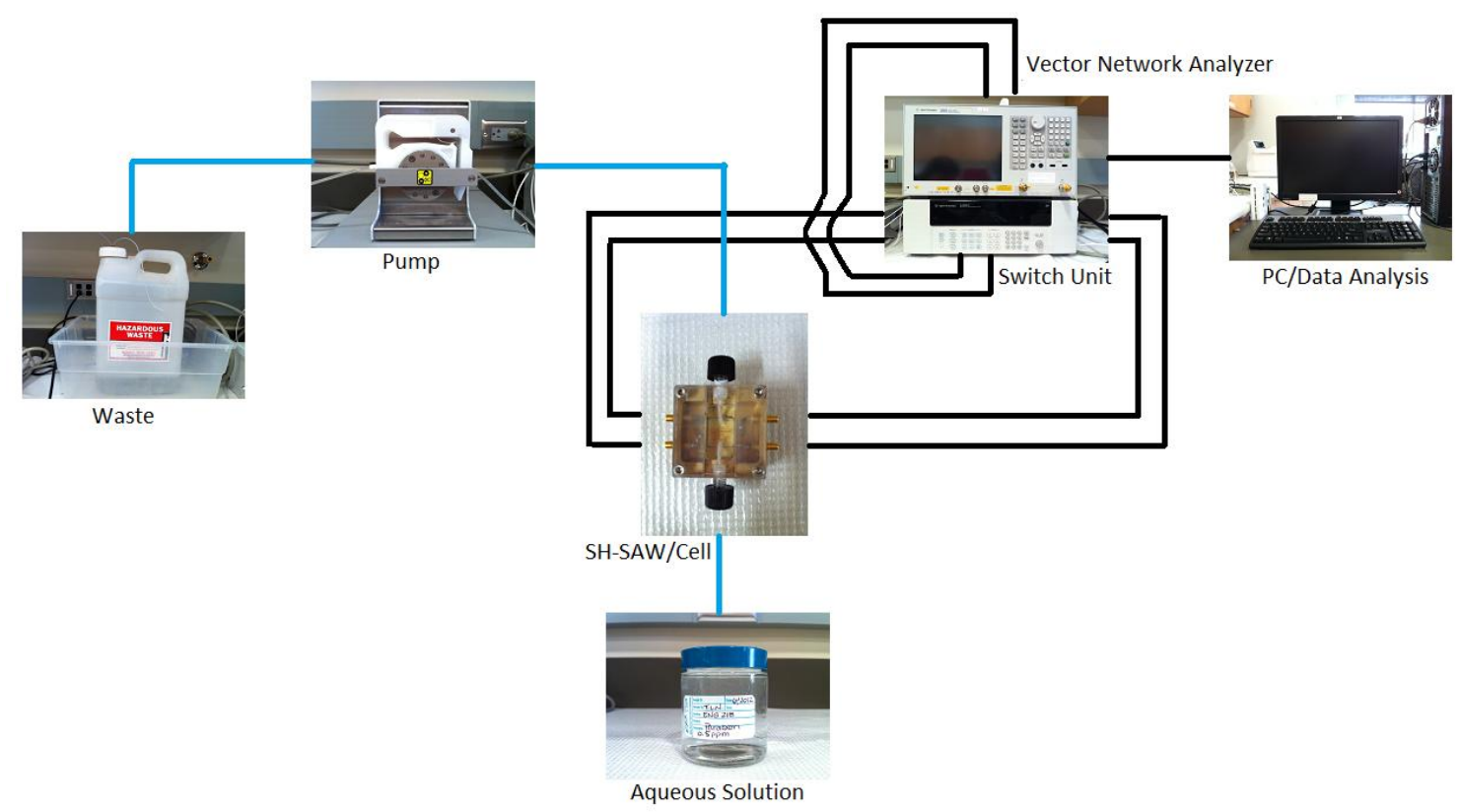

Figure 3.3: Diagram of the experimental setup including the flow system, flow cell, and network analyzer [24]

\subsubsection{SH-SAW Preparation}

The SH-SAW device needs to be prepared before it is used for sensing to improve the signal to noise ratio. In order to do so, the device must undergo several steps of filing to reduce reflections that can affect sensitivity and reproducibility of the device [24].

The edges of the SH-SAW device are beveled, using sandpaper, in order to scatter the waves reflected off the edges so that they are incoherent before they reach the output 
[26]. Then notches are created in between the contact pads of the newly sanded SH-SAW device to dissipate the bulk waves being generated by the IDTs. It is noted that all the above steps could be replaced by the use of an acoustic absorber at the edges of the crystal. The SH-SAW device is then cleaned with chloroform, acetone, and 2-propanol for three minutes each in an ultrasonic bath, DI water is used to rinse in between each cleaning solution. After it is dried with nitrogen gas, the bottom of the device is covered with electrical tape to absorb any remaining reflected bulk wave energy.

\subsubsection{Polymer Synthesis}

After the SH-SAW device is prepared, it is ready to be coated with a polymer. BPAPDMS was synthesized in the Marquette University Chemistry Lab using the procedure listed below [39]. This was based on the synthesis procedure of BPA-HMTS.

1. Turn on the hot plate and set an oil bath on top of the hot plate. Allow the temperature to set to $230^{\circ} \mathrm{C}$.

2. Using a pipette, add $10 \mathrm{~mL}$ of toluene to $40 \mathrm{~mL}$ vial.

3. Set the vial on a scale and tare.

4. Using a spatula, add $0.882 \mathrm{~g}$ of BPA.

5. Stir the mixture on a stir plate for 5 minutes at $400 \mathrm{rpm}$.

6. Using a pipette, add another $10 \mathrm{~mL}$ of toluene to the vial.

7. Using a spatula, add $1.5776 \mathrm{~g}$ of PDMS.

8. Stir the mixture on a stir plate at $400 \mathrm{rpm}$ for 5 minutes.

9. Measure the FTIR. A Si-H peak should be present.

10. Using a spatula, add $0.020 \mathrm{~g}$ of catalyst.

11. Stir the mixture on a stir plate at 400 rpm for 20 minutes in the oil bath. 
12. Measure the FTIR. The Si-H peak should have disappeared.

13. Using a spatula, add $0.4729 \mathrm{~g}$ of PDMS.

14. Stir the mixture in the oil bath for 20 minutes.

15. Measure the FTIR. The Si-H peak should be present.

16. Calculate the amount of excess catalyst required for the total amount of PDMS left. Add the PDMS to the mixture.

17. Transfer the sample to a three-neck flask and stir at 400rpm for 2 hours in the oil bath.

18. Measure the FTIR. The Si-H peak should be absent indicating that the reaction is complete.

19. Add activated charcoal to the mixture and stir it in the oil bath at $400 \mathrm{rpm}$ for 30 minutes.

20. Filter the mixture two times using 494 medium flow filter paper.

21. To calculate the \%wt of the mixture, start by weighing the empty vial $\left(w_{1}\right)$. Then weigh the vial with the mixture $\left(w_{2}\right)$ and calculate the difference to find the weight of the solution only $\left(w_{3}=w_{2}-w_{1}\right)$. Using the following equation:

$$
\% w t=\frac{\text { mass of } B P A-P D M S}{\text { mass of solution }} \times 100=\frac{\text { mass of } B P A-P D M S}{w_{3}} \times 100
$$

If the BPA-HMTS is prepared so that the solvent it fully evaporated, it can be prepared using another procedure. The preparation of PECH and PMMA were also done using the following steps. 
1. Calculate the amount of polymer and solvent needed to produce the \%wt needed.

2. Place a clean vial on a scale and tare the scale.

3. Add the required amount of polymer to a $20 \mathrm{~mL}$ vial.

4. Using a pipette, add the required amount of solvent to keep the \%wt.

For BPA-HMTS: chloroform

For PECH: chloroform

For PMMA: 2-ethoxyethyl acetate

5. Add a stir bar and seal the vial with parafilm.

6. For BPA-HMTS: Stir the polymer at 1000rpm at room temperature for 10 minutes.

7. For PECH: Stir the polymer at $1000 \mathrm{rpm}$ at a (nominal) temperature of $120^{\circ} \mathrm{C}$ for 2 hours and remove from the heat but continue stirring for another 22 hours.

8. For PMMA: Stir the polymer at $1000 \mathrm{rpm}$ at room temperature for 24 hours.

The polymer is evenly distributed onto the device using the spin coater. Table 3.1 represents the $\% \mathrm{wt}$, spin speed, and hold time needed to create a thickness for a specific polymer. 


\begin{tabular}{|c|c|c|c|}
\hline Polymer & Spin Speed (rpm) & Hold Time (s) & Average Film Thickness $(\mu \mathrm{m})$ \\
\hline BPA-HMTS 2.8\%wt & 3000 & 30 & 0.26 \\
\hline BPA-HMTS 4.6\%wt & 3000 & 30 & 0.51 \\
\hline BPA-PDMS 15\%wt & 4500 & 30 & 0.25 \\
\hline BPA-PDMS 20\%wt & 5000 & 30 & 0.50 \\
\hline PECH 1.05\%wt & 5000 & 30 & 0.25 \\
\hline PECH 2.10\%wt & 5000 & 30 & 0.39 \\
\hline PMMA14.95\%wt & 4500 & 50 & 0.48 \\
\hline
\end{tabular}

Table 3.1: Spin coating parameters to achieve various thicknesses for BPA-HMTS, BPA-PDMS, and PECH

\subsubsection{Phosphate Buffer Solution}

To prepare $0.1 \mathrm{M}$ of the Potassium Phosphate Buffer Solution (PBS), proceed as follows:

a) Monobasic Preparation

1. Using a burette, measure the required amount of $\mathrm{K}_{2} \mathrm{HPO}_{4}$.

2. Add degassed DI water to the $1000 \mathrm{~mL}$ mark.

b) Dibasic Preparation

1. Using a burette, measure the required amount of $\mathrm{KH}_{2} \mathrm{PO}_{4}$.

2. Add degassed DI water to the $1000 \mathrm{~mL}$ mark.

c) Combined Solution

1. Using a pipette add the required amount $\mathrm{K}_{2} \mathrm{HPO}_{4}$.

2. Add the required amount $\mathrm{KH}_{2} \mathrm{PO}_{4}$.

3. Add degassed DI water to the $2000 \mathrm{~mL}$ mark. 


\begin{tabular}{|c|c|c|}
\hline $\mathrm{pH}$ & $\begin{array}{c}\text { Volume of 0.1M } \\
\mathrm{K}_{2} \mathrm{HPO}_{4}\end{array}$ & $\begin{array}{c}\text { Volume of } 0.1 \mathrm{M} \\
\mathrm{KH}_{2} \mathrm{PO}_{4}\end{array}$ \\
\hline 6.2 & 13.2 & 80.8 \\
\hline 7.0 & 61.5 & 38.5 \\
\hline 8.0 & 94.0 & 6.0 \\
\hline
\end{tabular}

Table 3.2: Dilution chart to prepare different pH levels of PBS

\subsubsection{Concentrated Analyte Solution}

1. Using a pipette, measure $25.8 \mu \mathrm{L}, 25.7 \mu \mathrm{L}$, and $24.1 \mu \mathrm{L}$ of parathion, paraoxon, and parathion-methyl stock solutions respectively.

2. Add $3 \mu \mathrm{L}$ of methanol.

3. Cap, seal and stir at 1000rpm for 15 minutes.

4. Store at a temperature of $2-5^{\circ} \mathrm{C}$.

\subsubsection{Reference Solution}

1. Measure $960 \mathrm{~mL}$ of the buffer solution in a $1000 \mathrm{~mL}$ flask.

2. Add $1.1 \mathrm{~mL}$ of methanol to the flask using a pipette.

3. Add a stir bar and cover the flask with parafilm.

4. Stir at $1000 \mathrm{rpm}$ for 1 hour.

\subsubsection{Analyte Solution}

1. Using a burette, measure $120 \mathrm{~mL}$ of the buffer solution into a small jar.

2. Add $140 \mu \mathrm{L}$ of the concentrated analyte solution from the fridge.

3. Cap, seal, and stir at 600rpm for 2 hours. 


\begin{tabular}{|c|c|c|}
\hline Reference Solution $(\mathrm{mL})$ & Analyte Solution $(\mathrm{mL})$ & Concentration $(\mathrm{mg} / \mathrm{L})$ \\
\hline 121 & 5 & 0.5 \\
\hline 116 & 10 & 1.0 \\
\hline 111 & 15 & 1.5 \\
\hline 106 & 20 & 2.0 \\
\hline 101 & 25 & 2.5 \\
\hline 96 & 30 & 3.0 \\
\hline
\end{tabular}

Table 3.3: Dilution chart for the analyte solution

\subsection{Adding Interferents}

To test the devices under more realistic environmental conditions, interferents were added into the analyte solution to simulate groundwater. Relevant chemical and physical interferents in groundwater include soil/sediment (i.e. fine gravel, sand, clay), gasoline, and different $\mathrm{pH}$ levels.

The interferent used in this work was red clay which was grinded down to $75 \mu \mathrm{m}$ thick flakes. The clay was further grinded using a mortar and pestle. Each jar used in this measurement contained $20 \mathrm{mg}$ of ground clay.

The same experiments were also performed using the analyte solutions at different $\mathrm{pH}$ levels since groundwater has a $\mathrm{pH}$ range of 6 to 8.5 [43]. The $\mathrm{pH}$ levels that were tested were 6.2, 7.0, and 8.0. These were prepared using Table 3.2. 


\section{RESULTS AND SENSOR SIGNAL ANALYSIS}

\subsection{Introduction}

In the following chapter, the performance of the SH-SAW device coated with three partially chemically selective polymers (BPA-HMTS, BPA-PDMS, and PECH) for detection of three organophosphate pesticides (parathion, parathion-methyl, and paraoxon) in liquid phase is investigated. In order to compare and characterize the three selected polymer coatings, and also to build a sensor array, two thicknesses of each polymer were characterized and tested. Data which consists of frequency shifts for a given concentration are presented as a function of time. The performance of the three polymers is analyzed using the various sensitivity graphs. Sensitivity graphs normalized to the solubility of each analyte in liquid are also analyzed.

Steady state frequency responses and transient information (absorption time constant) of each coating/analyte pair were extracted from a basic exponential fit [39]. Since the sensor response follows different patterns for each coating/analyte pair, a sensor array is developed and analyzed for analyte identification and concentration determination utilizing both the steady state frequency responses and transient responses. A method using visual pattern recognition via radial plots will allow analyte classification and analyte concentration is determined through an algorithm based on the sample's "distance" from the known patterns $[44,45]$. Using this specific pattern recognition analysis, organophosphates are identified at low concentrations. The level of certainty in the identification process is quantified and the method of identification used is presented and discussed below. 
Stability of the polymers was also determined through multiple experiments. This was done to simulate measurements under real case applications which will be performed in groundwater. Groundwater contains many interferents. The sensors will be evaluated for reproducibility, sensitivity, selectivity/specificity as well as response to various physical and chemical contaminants (interferents such as different $\mathrm{pH}$ levels and sediments) and long term use. Colloids were added to the prepared solutions to simulate the presence of sediments. It is expected that the information presented in the following chapter can be used for the design and development of a sensor array that can be implemented for on-site monitoring of contaminated waters.

\subsection{Sensor Response and Discussion}

$\mathrm{LiTaO}_{3} \mathrm{SH}-\mathrm{SAW}$ devices were coated with two different thicknesses, $0.25 \mu \mathrm{m}$ and 0.5 $\mu \mathrm{m}$, of BPA-HMTS, BPA-PDMS, and PECH and exposed to six concentrations $(0.5 \mathrm{mg} / \mathrm{L}$ to $3.0 \mathrm{mg} / \mathrm{L})$ of parathion, parathion-methyl, and paraoxon, respectively. These thicknesses were chosen in order to investigate the effects of the coating thickness on sensor sensitivity. The frequency responses for each coated device to parathion are shown in Figures 4.1-4.6; Figures 4.7-4.12 show the responses to paraoxon; and Figures 4.134.18 show the responses to parathion-methyl. The concentration range that was chosen was based on EPA regulations for maximum contamination levels in groundwater [46]. All tests were performed continually under a 72 hour time frame for using freshly prepared solutions to allow proper parallel comparisons. All measurements were base line corrected for analytical purposes. It should also be noted that the figures were also scaled down with respect to largest response for easier comparison. 
As explained previously, the sensor responds when an analyte/polymer interaction occurs. The result of the interaction is mass loading and change in the coating viscoelastic properties. Mass loading affects the translational motion across the polymer and reduces the SAW propagation velocity. Viscoelastic effects cause film deformation associated with energy storage and power dissipation which effects both phase velocity and attenuation. In Figures 4.1-4.18, it is observed that the frequency shifts are a function of both mass loading and modulus change. The insertion loss is not shown however, because in most cases only a small response was observed. Therefore, the steady-state frequency shift and the response time (the time it takes for the response to reach $90 \%$ of its steady state value) of the frequency signal are considered the most useful input parameters for data analysis in the present case, and only those will be shown.

The figures show overall that the polymers, BPA-HMTS, PECH, and BPAPDMS, are most sensitive to least sensitive to parathion, parathion-methyl, and paraoxon, respectively. The polymers with a thickness of $0.5 \mu \mathrm{m}$ also show a higher response than the $0.25 \mu \mathrm{m}$-thick polymers. This is due to the larger volume of the polymer which allows more absorption of analyte into the polymer. This leads to a more pronounced effect of mass loading and modulus change. It is also observed that the $0.5 \mu \mathrm{m}$-thick BPA-PDMS coating shows positive frequency shifts for all analytes. Due to the rubbery nature of this polymer, strong viscoelastic effects take place, resulting in a large phase lag across the thickness of the coating. This phenomenon is further analyzed in Section 4.3.

The frequency shift changes almost linearly with the analyte concentration within the measured concentration range. Due to the small variations in analyte concentration, the frequency shift will vary slightly from sample to sample. Repeated experiments were 
conducted to improve measurement accuracy and reproducibility. It is also observed that the frequency response is reversible (returning back to the baseline value after the analyte is removed) which indicates that the analyte/polymer interaction is largely physical in nature and the sensor is reusable.

It can be seen that, for BPA-HMTS and BPA-PDMS, the response times for the analytes can take relatively long (up to 120 minutes). The presented response times are only valid for this sensor system. Response times are functions of sensor system (the flow rate and cell volume), coating properties, and sorption kinetics. For this work, the flow rate was chosen to be $.72 \mathrm{~mL} / \mathrm{min}$ and the cell volume is approximately $.134 \mathrm{~mL}$. A higher flow rate would allow the cell to be filled/emptied quicker but as a result there would be turbulence in the cell and noise would be introduced to the system. It is also important to observe the sorption kinetics. For polymeric films, the response times for polymers in the glassy state have slower response times and the polymer in the rubbery state exhibit faster response times. It has been shown that the response time is also affected by the pore size of the polymer and the analyte molecule dimension. The more porous the coating and the smaller the analyte molecule size, the faster the response time [21]. The rate of analyte transport can also have an effect on response times [21]. Often, the rate of analyte transport into the polymer is limited by the analyte concentration of the solution. Therefore, if there are different analytes but identical analyte concentrations, similar initial slopes in the frequency response will be observed. However, if one analyte has a much larger partition coefficient, then the response curve will take a much longer time to reach its steady state value. At first approximation, the response time will often be 
proportional to the partition coefficient of an analyte. This could explain the fast to slow order of the response times of PECH, BPA-PDMS, and BPA-HMTS, respectively.

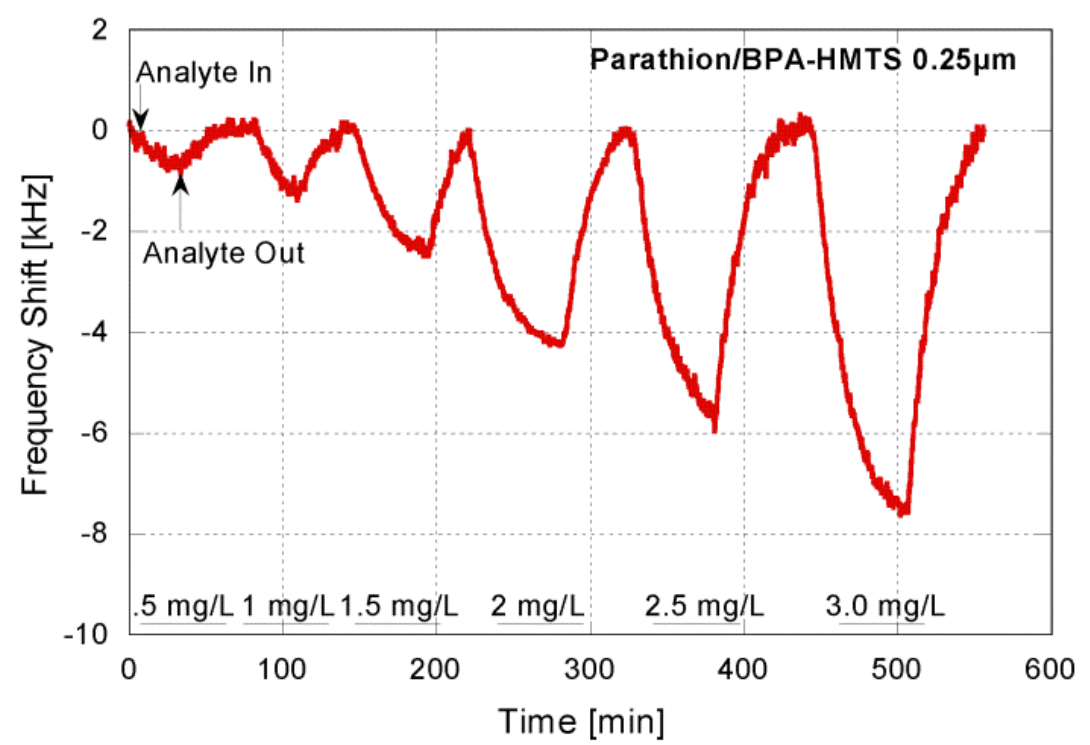

Figure 4.1: Measured change in frequency shift upon exposure of the BPA-HMTS 0.25 $\mu \mathrm{m}$ coated device to $0.5 \mathrm{mg} / \mathrm{L}, 1.0 \mathrm{mg} / \mathrm{L}, 1.5 \mathrm{mg} / \mathrm{L}, 2.0 \mathrm{mg} / \mathrm{L}, 2.5 \mathrm{mg} / \mathrm{L}$, and $3.0 \mathrm{mg} / \mathrm{L}$ of parathion.

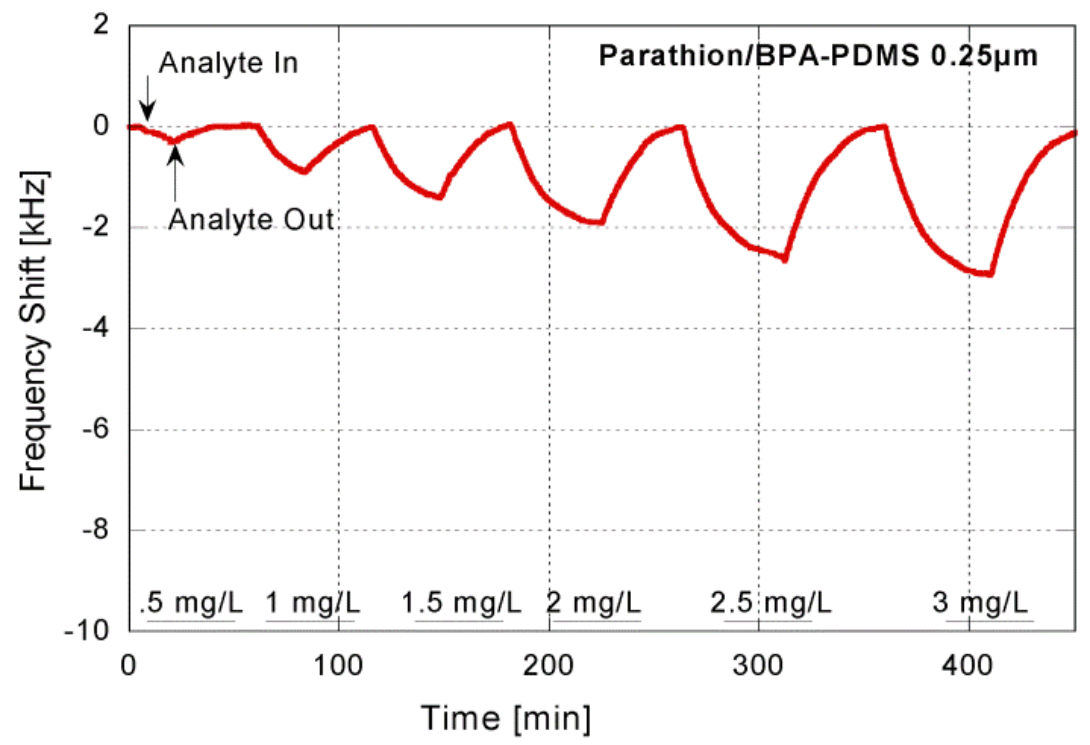

Figure 4.2: Measured change in frequency shift upon exposure of the BPA-PDMS $0.25 \mu \mathrm{m}$ coated device to $0.5 \mathrm{mg} / \mathrm{L}, 1.0 \mathrm{mg} / \mathrm{L}, 1.5 \mathrm{mg} / \mathrm{L}, 2.0 \mathrm{mg} / \mathrm{L}, 2.5 \mathrm{mg} / \mathrm{L}$, and $3.0 \mathrm{mg} / \mathrm{L}$ of parathion. 


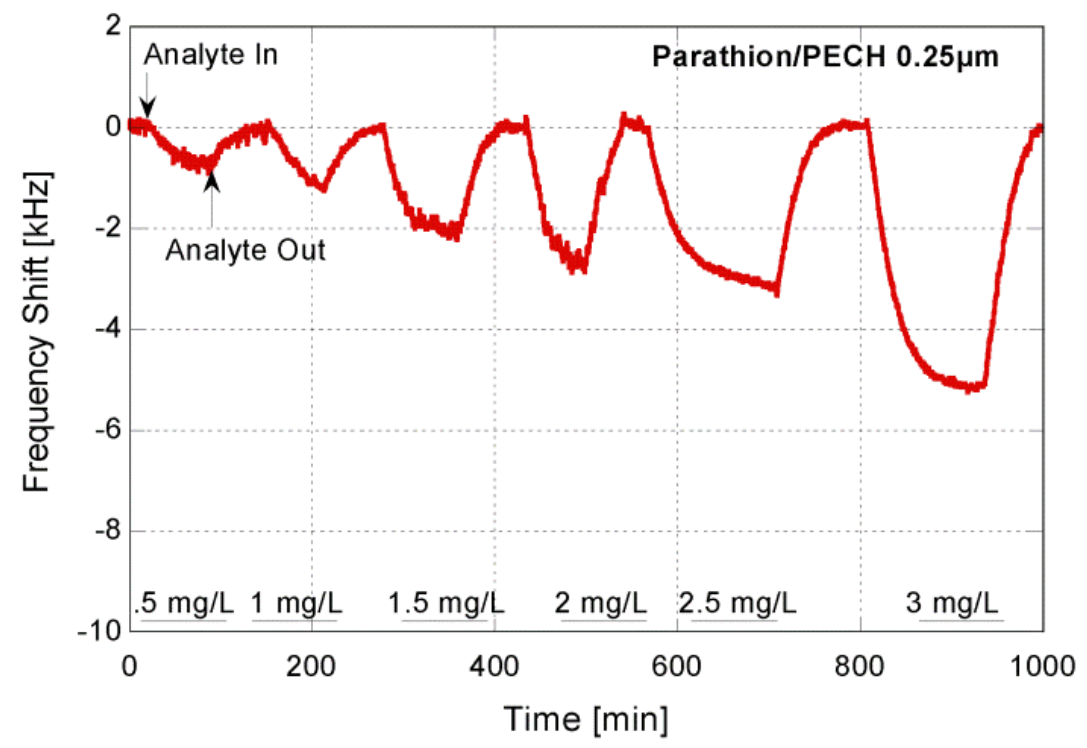

Figure 4.3: Measured change in frequency shift upon exposure of the PECH $0.25 \mu \mathrm{m}$ coated device to $0.5 \mathrm{mg} / \mathrm{L}, 1.0 \mathrm{mg} / \mathrm{L}, 1.5 \mathrm{mg} / \mathrm{L}, 2.0 \mathrm{mg} / \mathrm{L}, 2.5 \mathrm{mg} / \mathrm{L}$, and $3.0 \mathrm{mg} / \mathrm{L}$ of parathion.

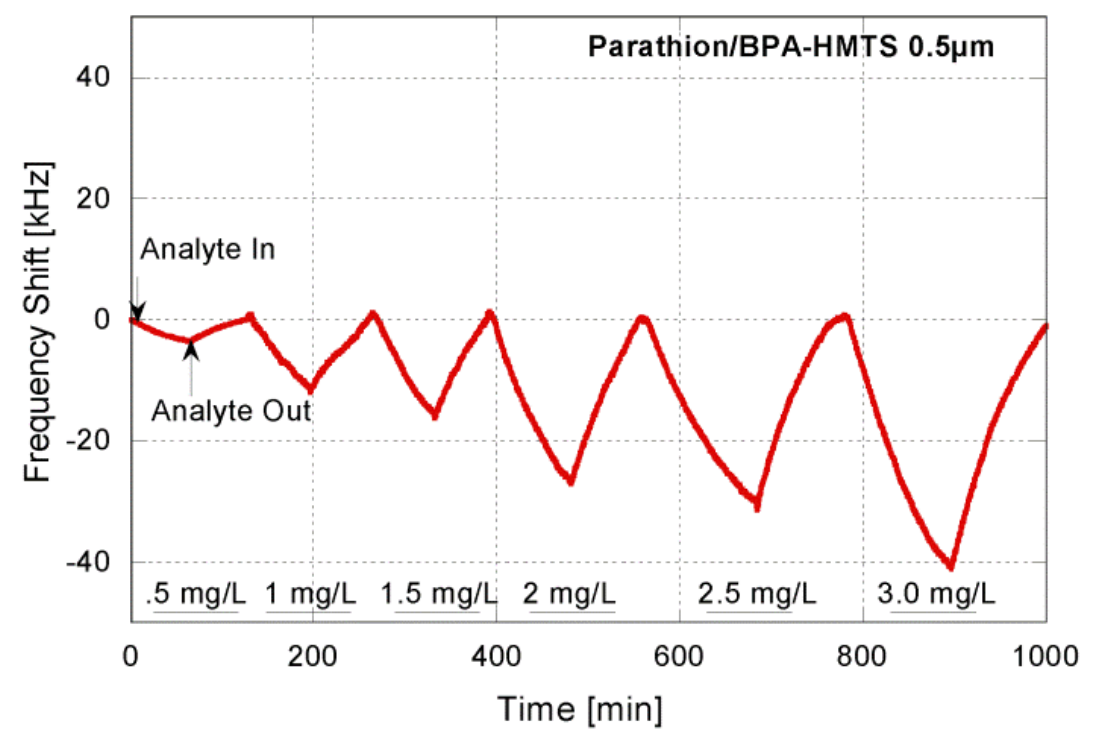

Figure 4.4: Measured change in frequency shift upon exposure of the BPA-HMTS 0.5 $\mu \mathrm{m}$ coated device to $0.5 \mathrm{mg} / \mathrm{L}, 1.0 \mathrm{mg} / \mathrm{L}, 1.5 \mathrm{mg} / \mathrm{L}, 2.0 \mathrm{mg} / \mathrm{L}, 2.5 \mathrm{mg} / \mathrm{L}$, and $3.0 \mathrm{mg} / \mathrm{L}$ of parathion. This coating/analyte pair shows the largest response. 


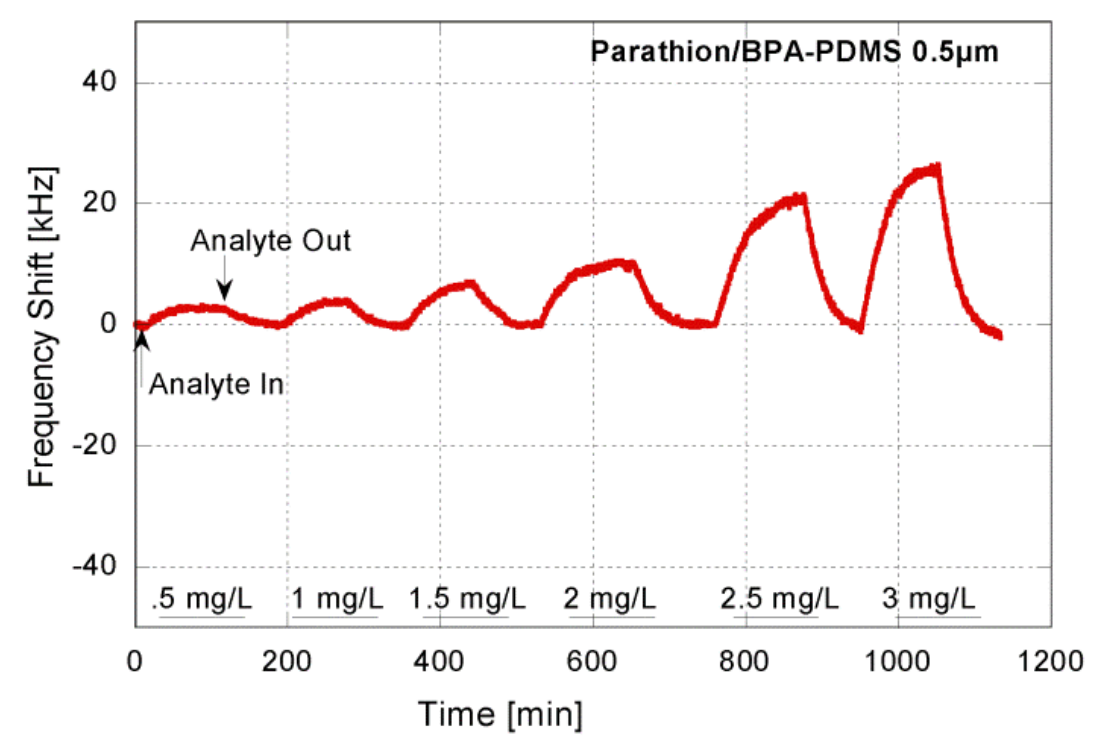

Figure 4.5: Measured change in frequency shift upon exposure of the BPA-PDMS 0.5 $\mu \mathrm{m}$ coated device to $0.5 \mathrm{mg} / \mathrm{L}, 1.0 \mathrm{mg} / \mathrm{L}, 1.5 \mathrm{mg} / \mathrm{L}, 2.0 \mathrm{mg} / \mathrm{L}, 2.5 \mathrm{mg} / \mathrm{L}$, and $3.0 \mathrm{mg} / \mathrm{L}$ of parathion. Note that the frequency shift is positive which will be explained by the polymer viscoelasticity effect in Section 4.3.

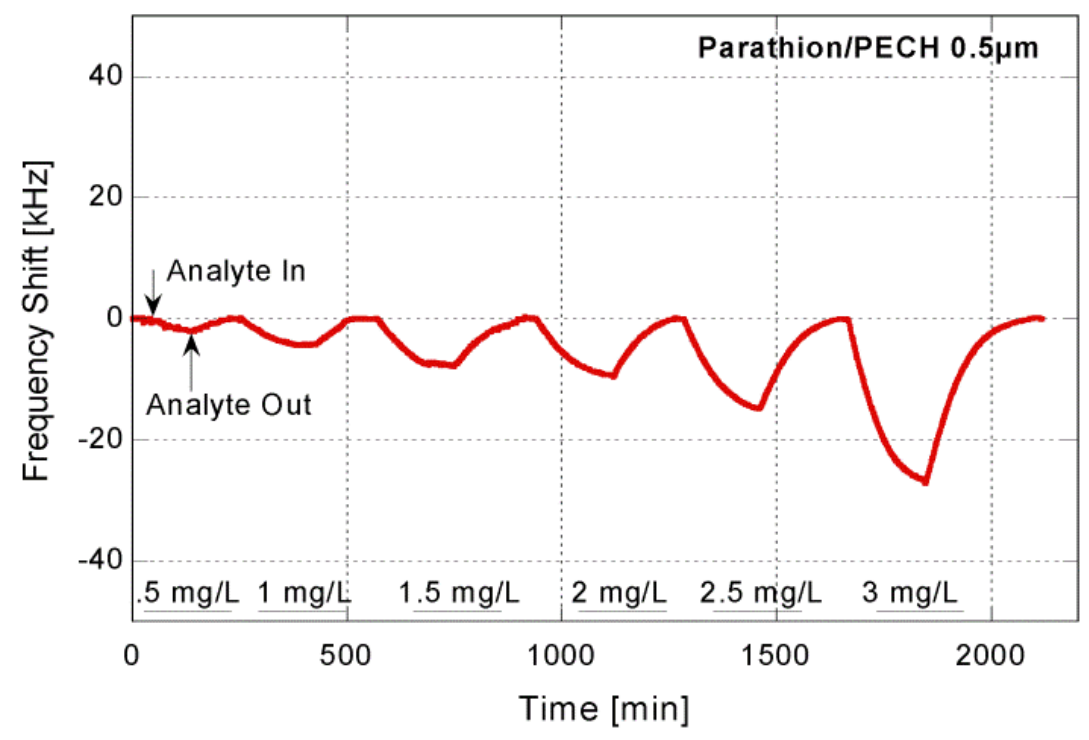

Figure 4.6: Measured change in frequency shift upon exposure of the PECH $0.5 \mu \mathrm{m}$ coated device to $0.5 \mathrm{mg} / \mathrm{L}, 1.0 \mathrm{mg} / \mathrm{L}, 1.5 \mathrm{mg} / \mathrm{L}, 2.0 \mathrm{mg} / \mathrm{L}, 2.5 \mathrm{mg} / \mathrm{L}$, and $3.0 \mathrm{mg} / \mathrm{L}$ of parathion. 


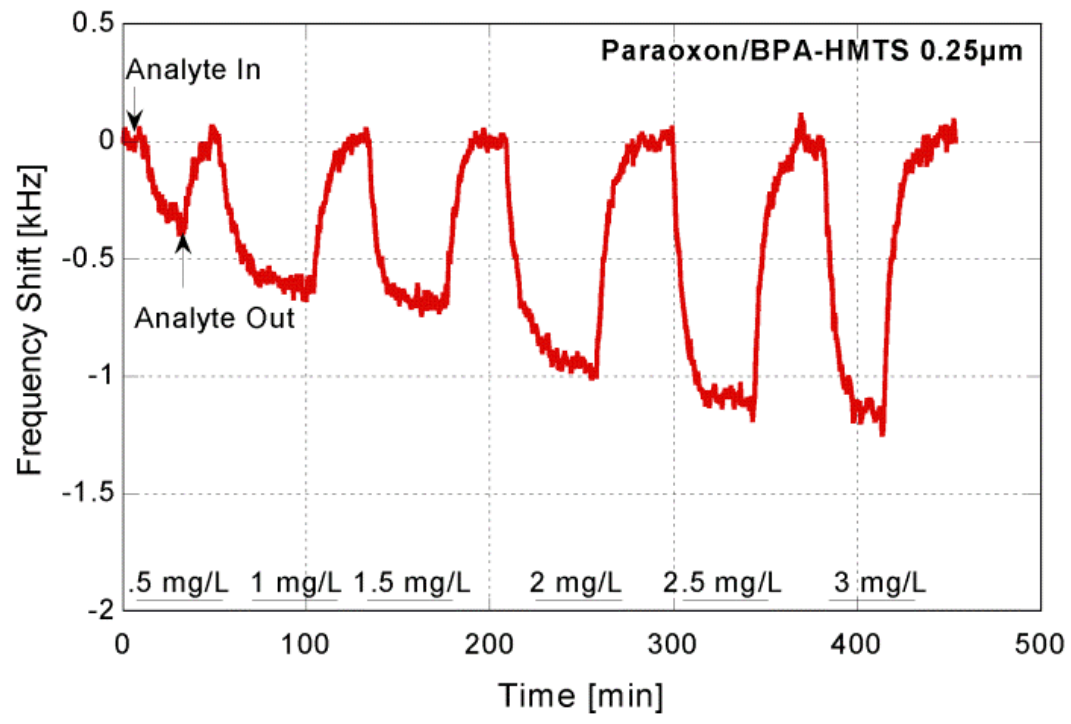

Figure 4.7: Measured change in frequency shift upon exposure of the BPA-HMTS $0.25 \mu \mathrm{m}$ coated device to $0.5 \mathrm{mg} / \mathrm{L}, 1.0 \mathrm{mg} / \mathrm{L}, 1.5 \mathrm{mg} / \mathrm{L}, 2.0 \mathrm{mg} / \mathrm{L}, 2.5 \mathrm{mg} / \mathrm{L}$, and $3.0 \mathrm{mg} / \mathrm{L}$ of paraoxon. Compared to the previous figure, the frequency axis is scaled down by a factor of 40.

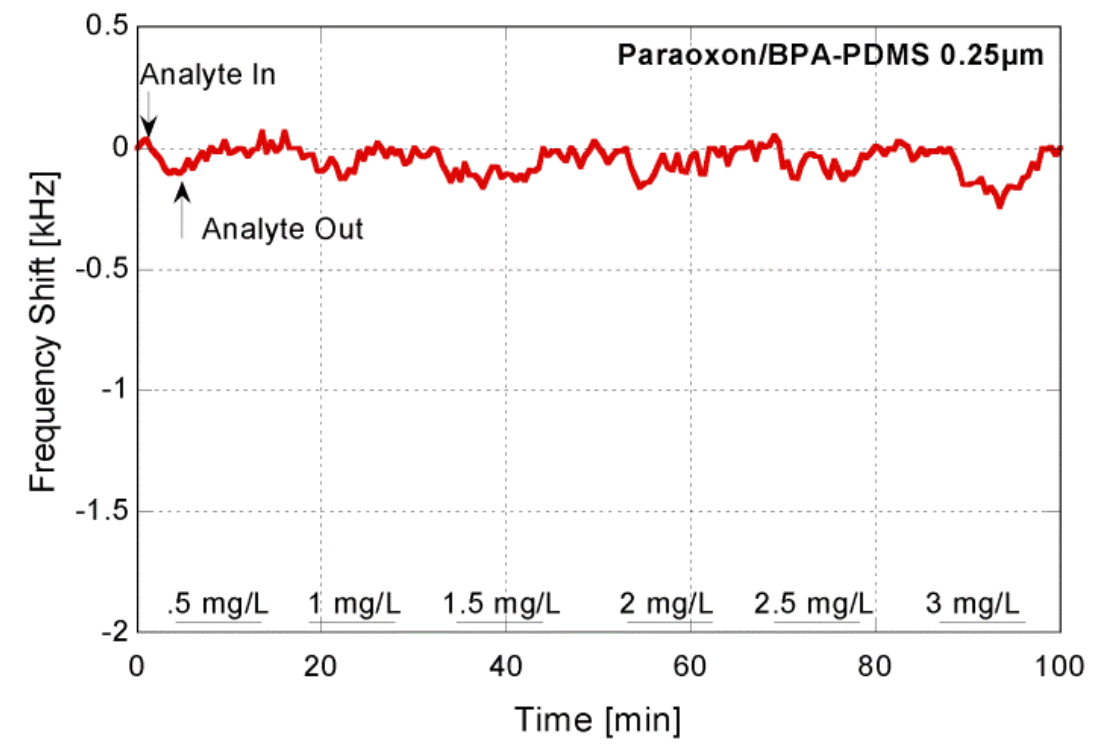

Figure 4.8: Measured change in frequency shift upon exposure of the BPA-PDMS $0.25 \mu \mathrm{m}$ coated device to $0.5 \mathrm{mg} / \mathrm{L}, 1.0 \mathrm{mg} / \mathrm{L}, 1.5 \mathrm{mg} / \mathrm{L}, 2.0 \mathrm{mg} / \mathrm{L}, 2.5 \mathrm{mg} / \mathrm{L}$, and 3.0mg/L of paraoxon. 


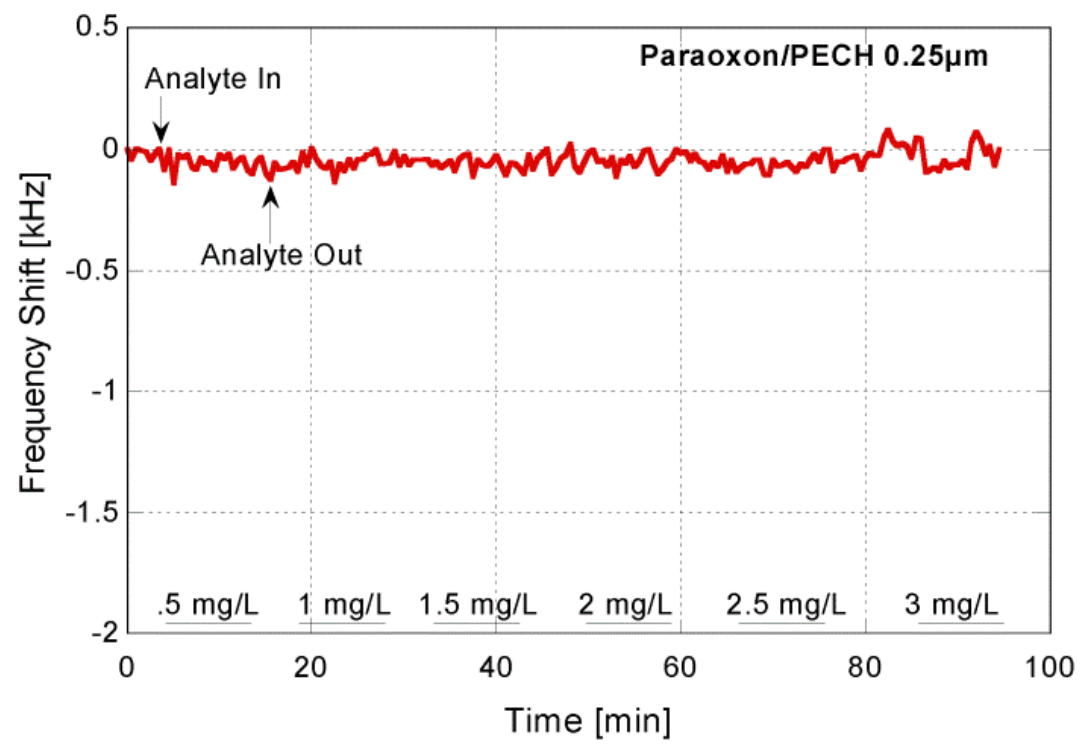

Figure 4.9: Measured change in frequency shift upon exposure of the PECH $0.25 \mu \mathrm{m}$ coated device to $0.5 \mathrm{mg} / \mathrm{L}, 1.0 \mathrm{mg} / \mathrm{L}, 1.5 \mathrm{mg} / \mathrm{L}, 2.0 \mathrm{mg} / \mathrm{L}, 2.5 \mathrm{mg} / \mathrm{L}$, and $3.0 \mathrm{mg} / \mathrm{L}$ of paraoxon.

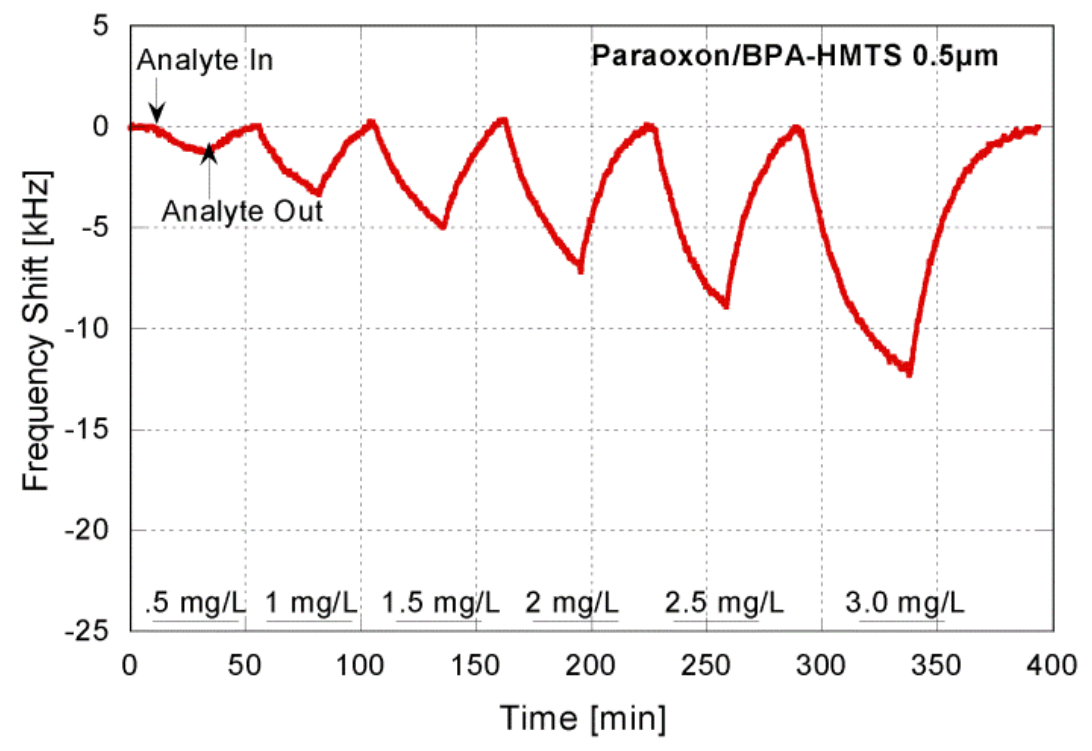

Figure 4.10: Measured change in frequency shift upon exposure of the BPA-HMTS 0.5 $\mu \mathrm{m}$ coated device to $0.5 \mathrm{mg} / \mathrm{L}, 1.0 \mathrm{mg} / \mathrm{L}, 1.5 \mathrm{mg} / \mathrm{L}, 2.0 \mathrm{mg} / \mathrm{L}, 2.5 \mathrm{mg} / \mathrm{L}$, and $3.0 \mathrm{mg} / \mathrm{L}$ of paraoxon. The frequency axis is scaled down by a factor of 3 compared to Figures 4.4-4.6. 


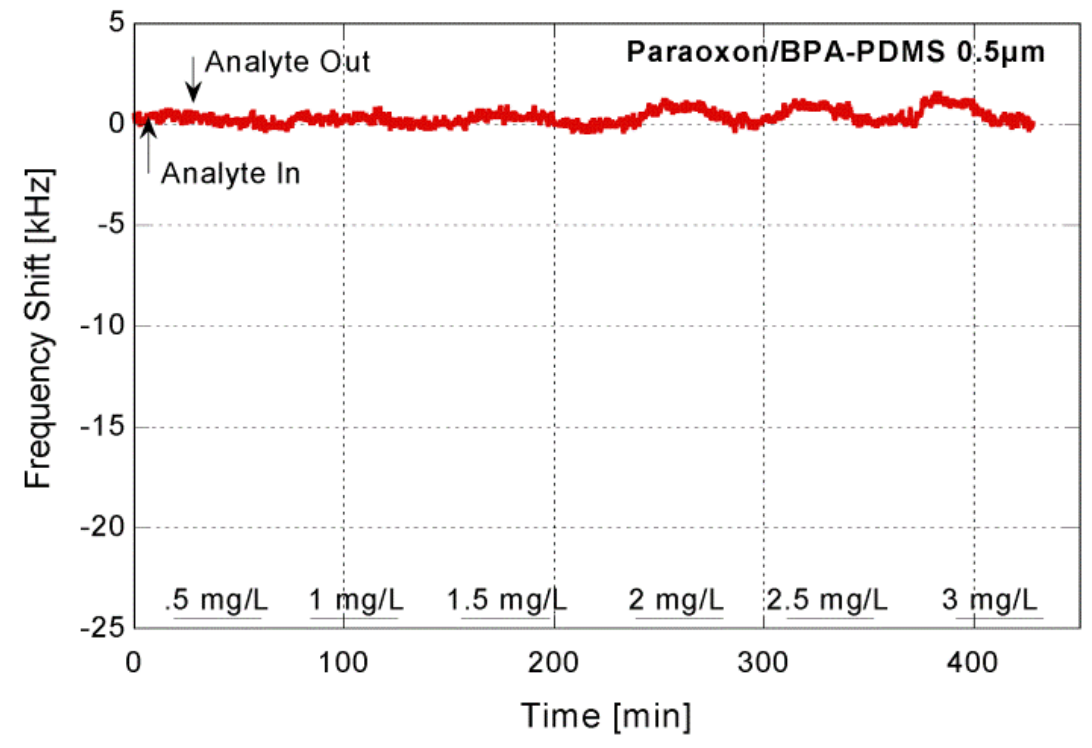

Figure 4.11: Measured change in frequency shift upon exposure of the BPA-PDMS 0.5 $\mu \mathrm{m}$ coated device to $0.5 \mathrm{mg} / \mathrm{L}, 1.0 \mathrm{mg} / \mathrm{L}, 1.5 \mathrm{mg} / \mathrm{L}, 2.0 \mathrm{mg} / \mathrm{L}, 2.5 \mathrm{mg} / \mathrm{L}$, and $3.0 \mathrm{mg} / \mathrm{L}$ of paraoxon.

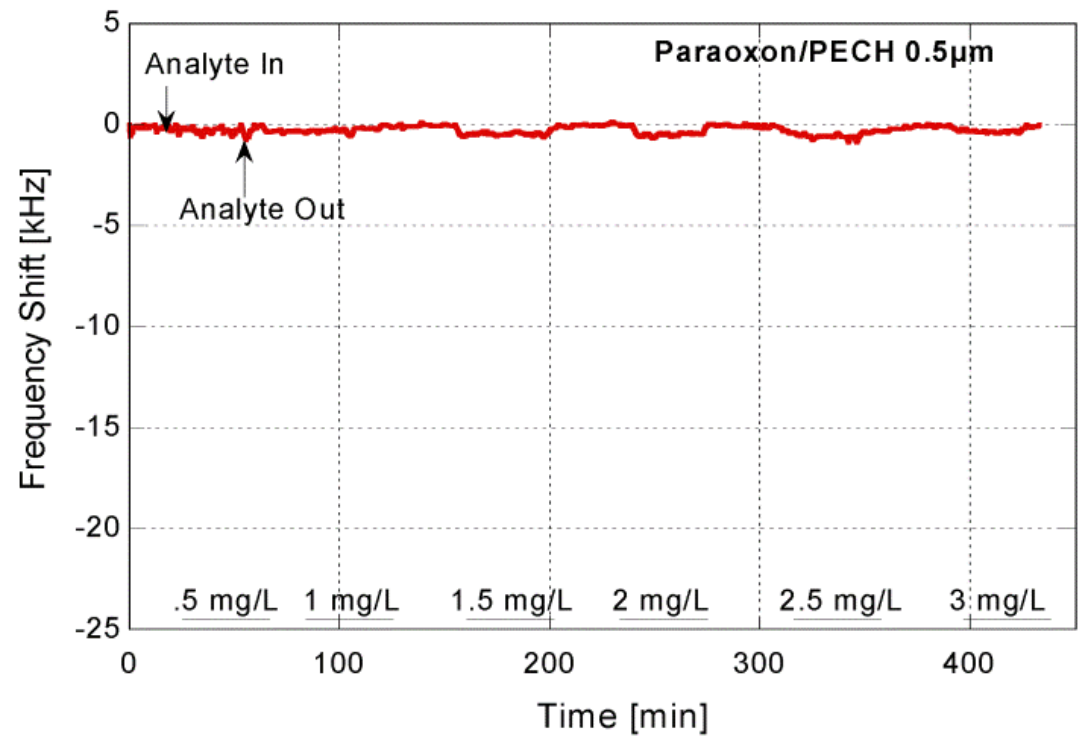

Figure 4.12: Measured change in frequency shift upon exposure of the PECH $0.5 \mu \mathrm{m}$ coated device to $0.5 \mathrm{mg} / \mathrm{L}, 1.0 \mathrm{mg} / \mathrm{L}, 1.5 \mathrm{mg} / \mathrm{L}, 2.0 \mathrm{mg} / \mathrm{L}, 2.5 \mathrm{mg} / \mathrm{L}$, and $3.0 \mathrm{mg} / \mathrm{L}$ of paraoxon. 


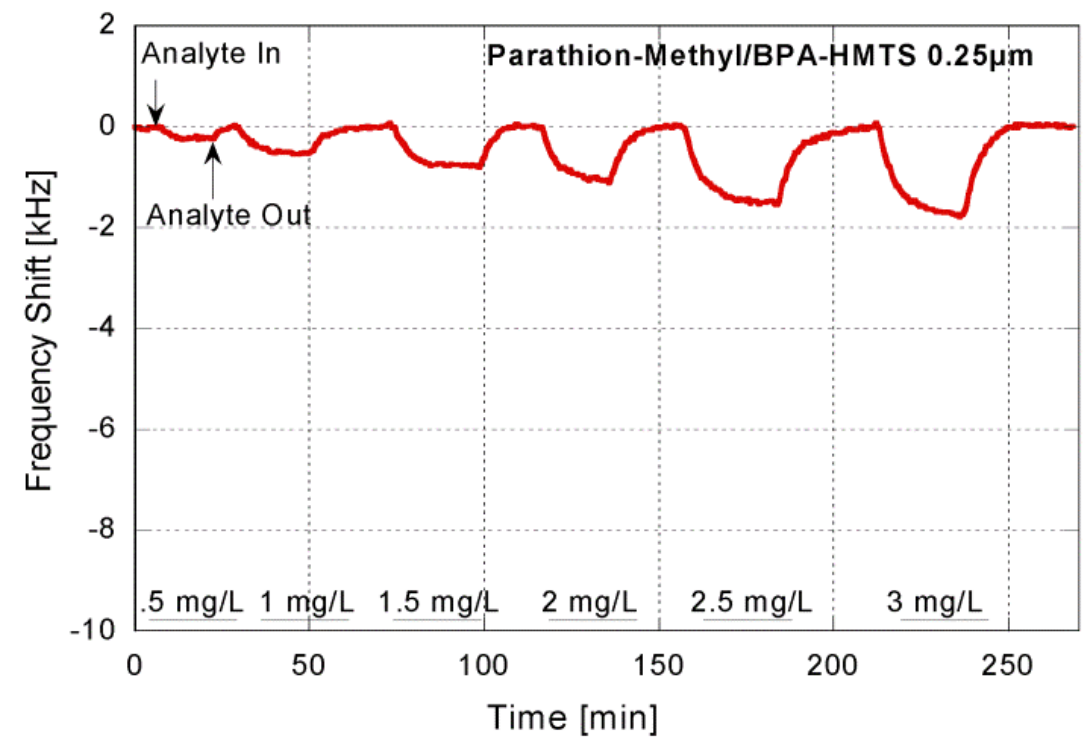

Figure 4.13: Measured change in frequency shift upon exposure of the BPA-HMTS 0.25 $\mu \mathrm{m}$ coated device to $0.5 \mathrm{mg} / \mathrm{L}, 1.0 \mathrm{mg} / \mathrm{L}, 1.5 \mathrm{mg} / \mathrm{L}, 2.0 \mathrm{mg} / \mathrm{L}, 2.5 \mathrm{mg} / \mathrm{L}$, and $3.0 \mathrm{mg} / \mathrm{L}$ of parathion-methyl. The frequency axis is scaled down by a factor of 8.

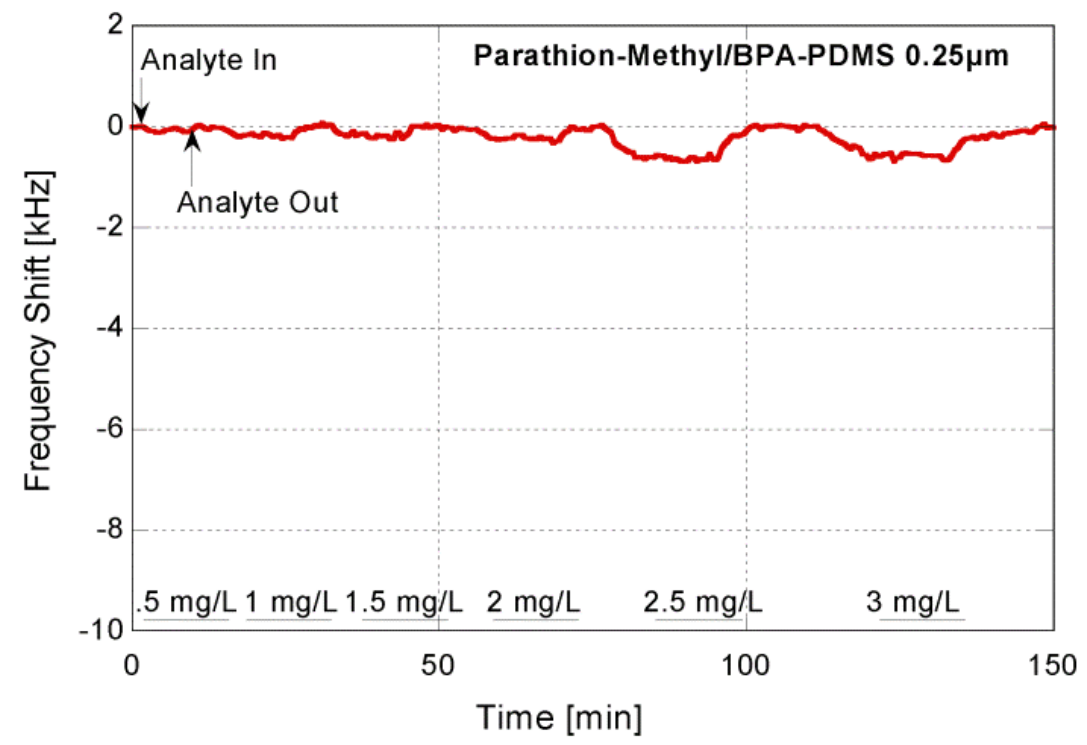

Figure 4.14: Measured change in frequency shift upon exposure of the BPA-PDMS 0.25 $\mu \mathrm{m}$ coated device to $0.5 \mathrm{mg} / \mathrm{L}, 1.0 \mathrm{mg} / \mathrm{L}, 1.5 \mathrm{mg} / \mathrm{L}, 2.0 \mathrm{mg} / \mathrm{L}, 2.5 \mathrm{mg} / \mathrm{L}$, and $3.0 \mathrm{mg} / \mathrm{L}$ of parathion-methyl. 


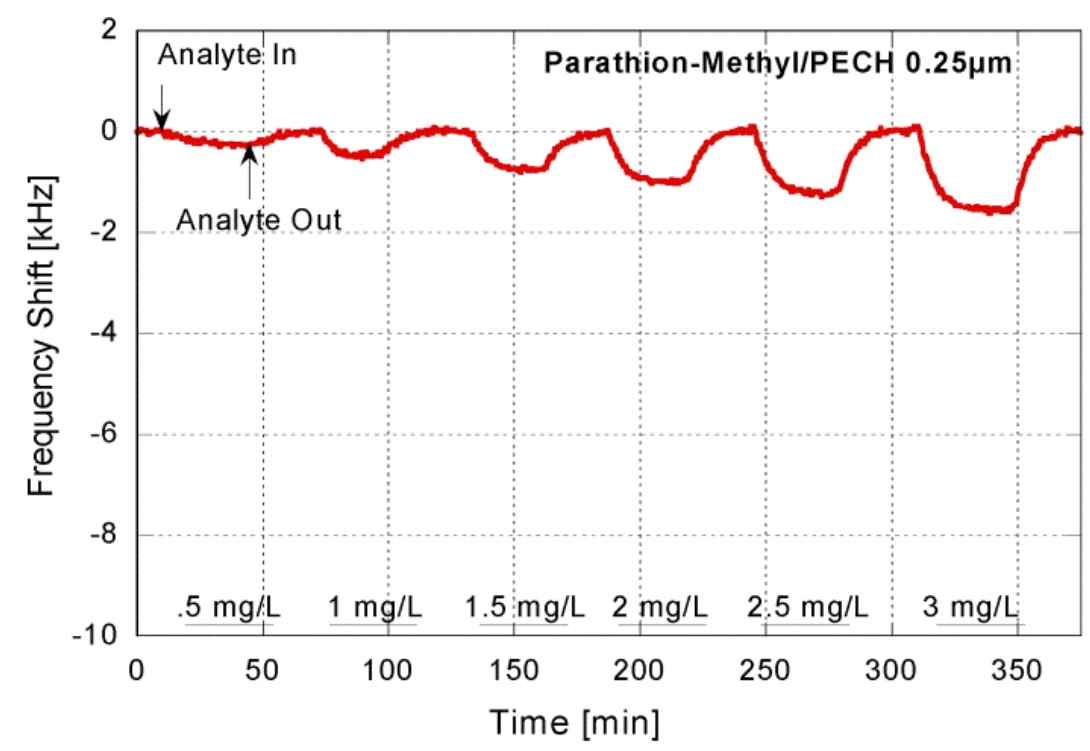

Figure 4.15: Measured change in frequency shift upon exposure of the PECH 0.25 $\mu \mathrm{m}$ coated device to $0.5 \mathrm{mg} / \mathrm{L}, 1.0 \mathrm{mg} / \mathrm{L}, 1.5 \mathrm{mg} / \mathrm{L}, 2.0 \mathrm{mg} / \mathrm{L}, 2.5 \mathrm{mg} / \mathrm{L}$, and $3.0 \mathrm{mg} / \mathrm{L}$ of parathion-methyl.

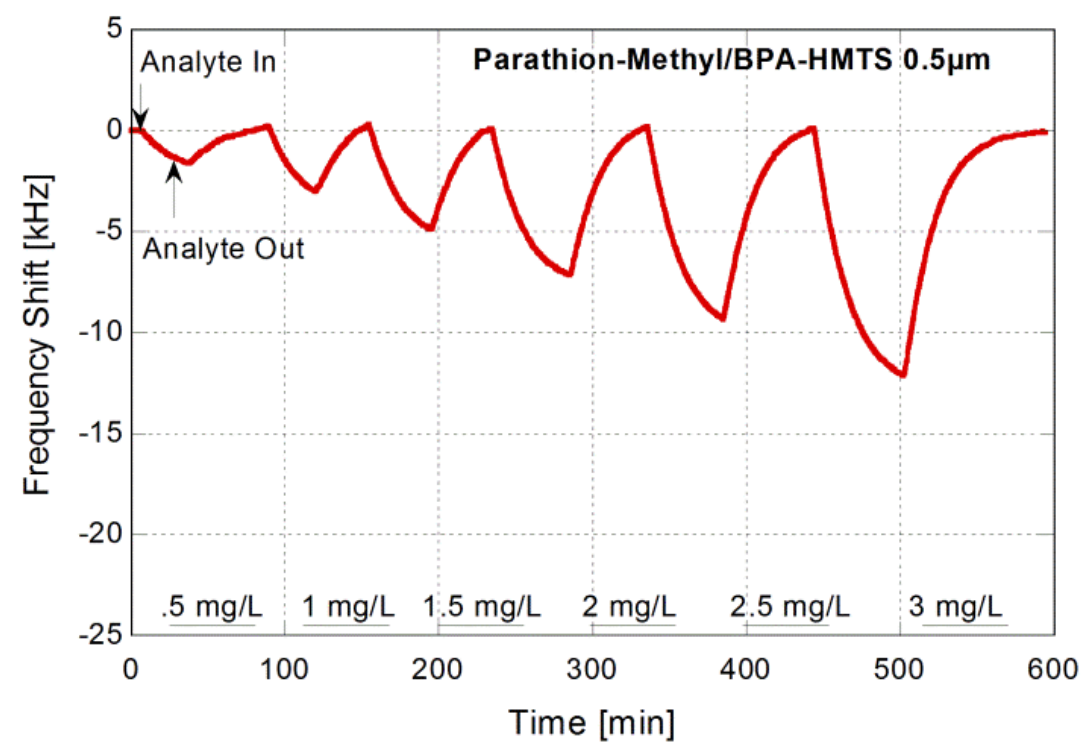

Figure 4.16: Measured change in frequency shift upon exposure of the BPA-HMTS 0.5 $\mu \mathrm{m}$ coated device to $0.5 \mathrm{mg} / \mathrm{L}, 1.0 \mathrm{mg} / \mathrm{L}, 1.5 \mathrm{mg} / \mathrm{L}, 2.0 \mathrm{mg} / \mathrm{L}, 2.5 \mathrm{mg} / \mathrm{L}$, and $3.0 \mathrm{mg} / \mathrm{L}$ of parathion-methyl. The frequency axis is scaled down by a factor of 3. 


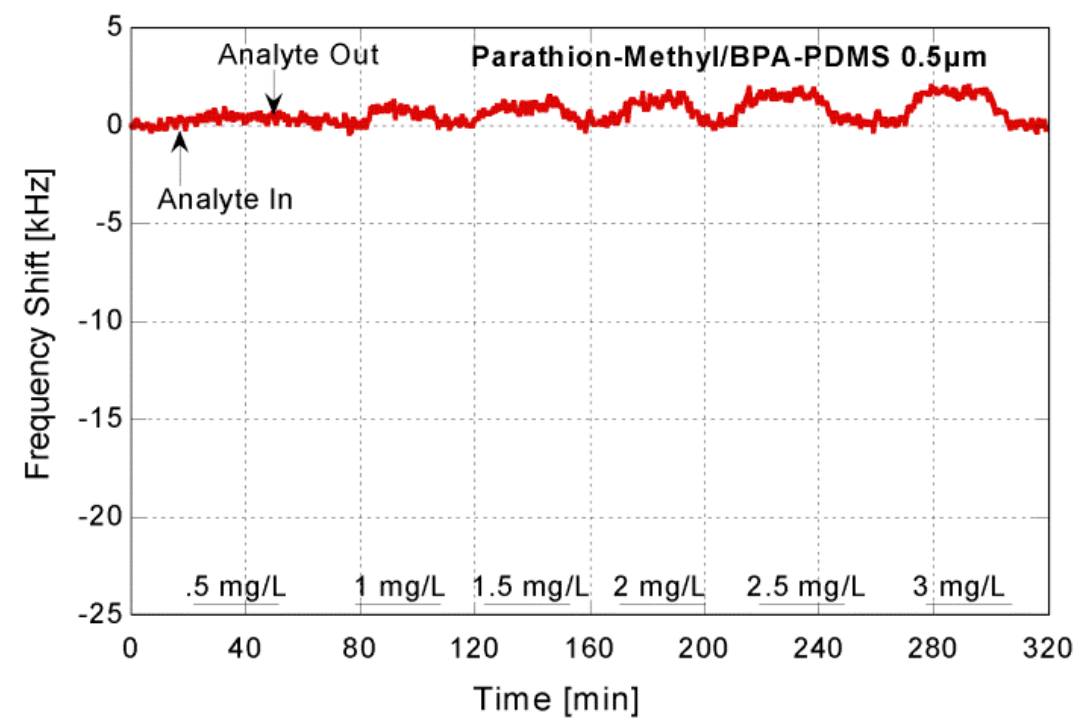

Figure 4.17: Measured change in frequency shift upon exposure of the BPA-PDMS 0.5 $\mu \mathrm{m}$ coated device to $0.5 \mathrm{mg} / \mathrm{L}, 1.0 \mathrm{mg} / \mathrm{L}, 1.5 \mathrm{mg} / \mathrm{L}, 2.0 \mathrm{mg} / \mathrm{L}, 2.5 \mathrm{mg} / \mathrm{L}$, and $3.0 \mathrm{mg} / \mathrm{L}$ of parathion-methyl.

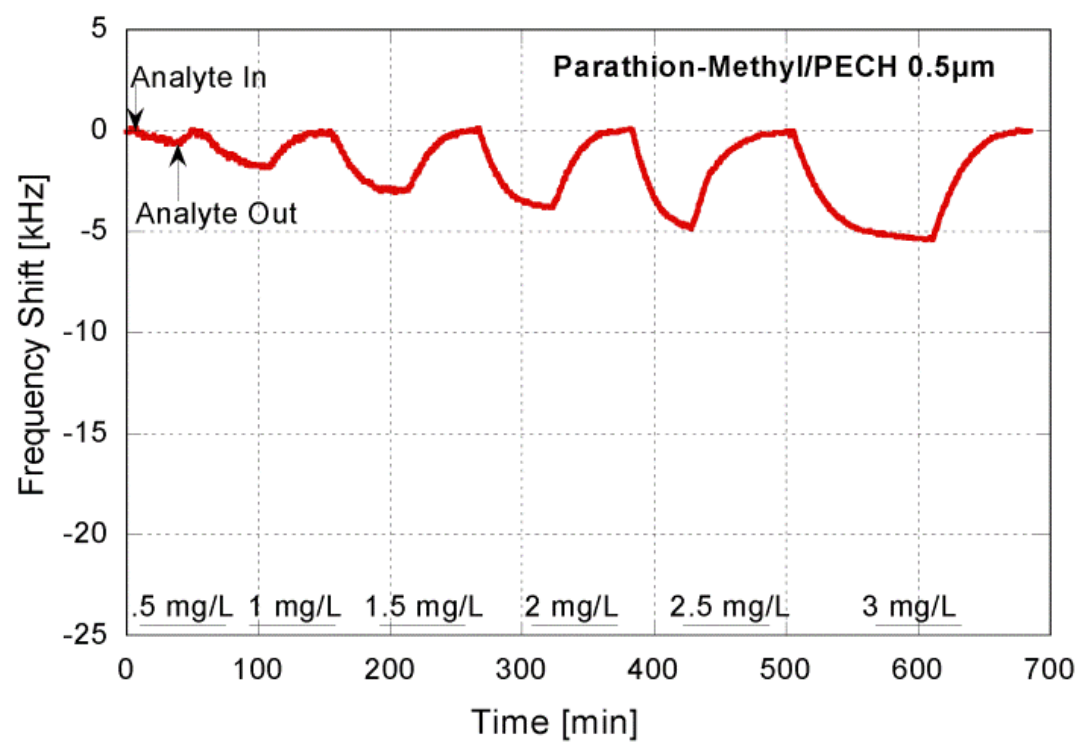

Figure 4.18: Measured change in frequency shift upon exposure of the PECH $0.5 \mu \mathrm{m}$ coated device to $0.5 \mathrm{mg} / \mathrm{L}, 1.0 \mathrm{mg} / \mathrm{L}, 1.5 \mathrm{mg} / \mathrm{L}, 2.0 \mathrm{mg} / \mathrm{L}, 2.5 \mathrm{mg} / \mathrm{L}$, and $3.0 \mathrm{mg} / \mathrm{L}$ of parathion-methyl. 
The three polymers, BPA-HMTS, BPA-PDMS, and PECH were analyzed and compared as chemically sensitive coatings for the detection of parathion, parathionmethyl, and paraoxon. It was observed that all of the polymers are sensitive to the organophosphate pesticides but each at their own degree. In order to compare the polymers affinities for the analytes and to evaluate how to best combine them in a sensor array, the frequency sensitivities are first calculated using

$$
S=\frac{\Delta f}{\Delta C}
$$

where $\Delta f$ represents the frequency shift and $\Delta C$ is the change in concentration. The frequency shift is taken from the steady state value of the frequency response when the device is exposed to a given analyte concentration. The frequency shift is plotted as a function of analyte concentration, and the data are modeled with a linear fit. The frequency sensitivity is determined from the slope of the fit. The sensitivity plots are shown in Figures 4.19-4.24. The sensitivity data is summarized in Table A.1 in the Appendix. It is important to note here that not all of the data will perfectly fall into a linear fit. As previously stated, this is due to inaccuracy in sample concentration, and also viscoelastic effects. The latter will be explained in Section 4.3. The sensitivity data will help in the design of the sensor array. 


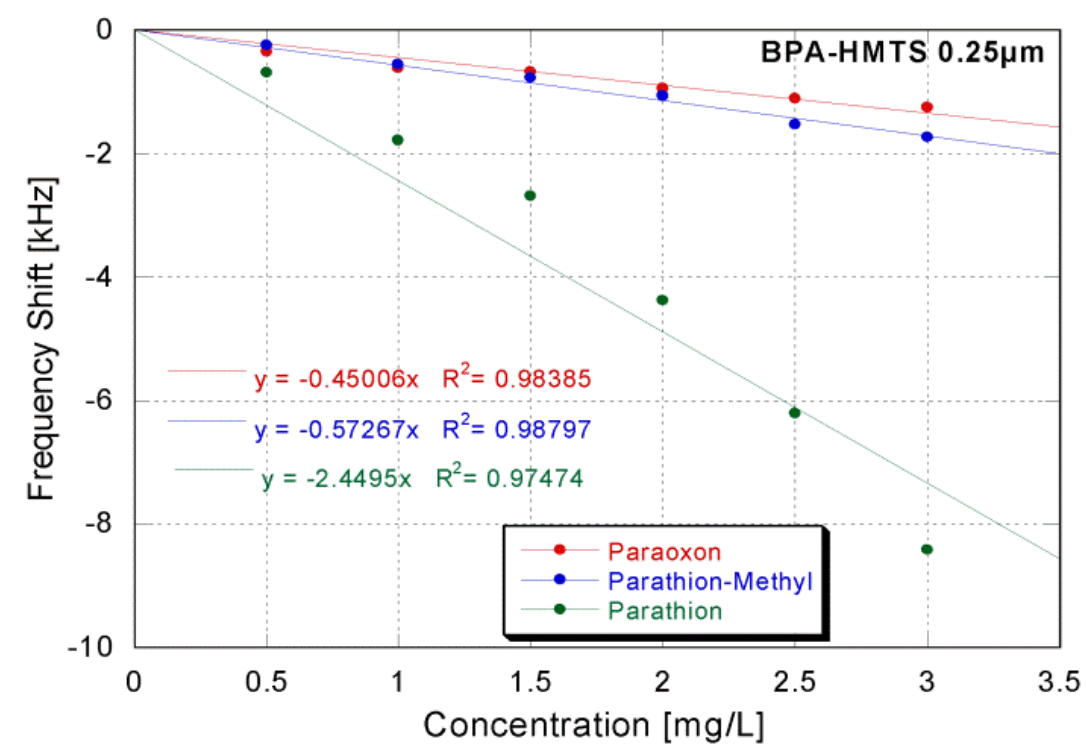

Figure 4.19: Sensitivity curve for the detection of $0.5 \mathrm{mg} / \mathrm{L}$ to $3.0 \mathrm{mg} / \mathrm{L}$ of parathion, parathionmethyl, and paraoxon using a guided SH-SAW device coated with $0.25 \mu \mathrm{m}$ of BPA-HMTS.

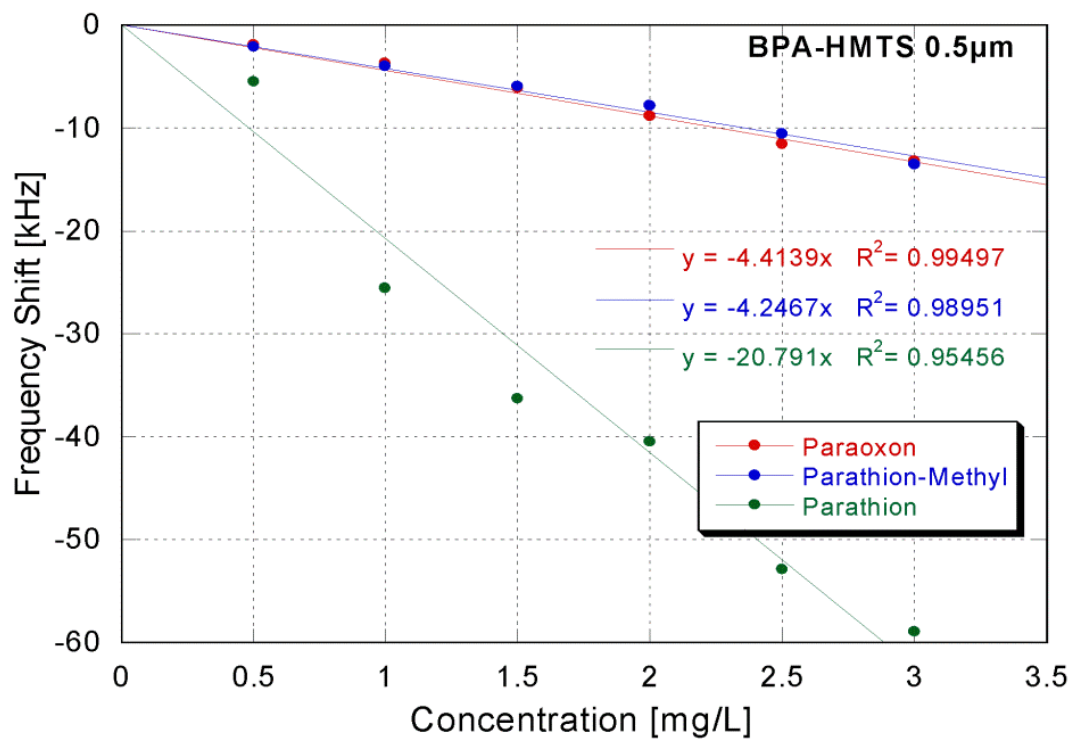

Figure 4.20: Sensitivity curve for the detection of $0.5 \mathrm{mg} / \mathrm{L}$ to $3.0 \mathrm{mg} / \mathrm{L}$ of parathion, parathionmethyl, and paraoxon using a guided SH-SAW device coated with $0.5 \mu \mathrm{m}$ of BPA-HMTS. 


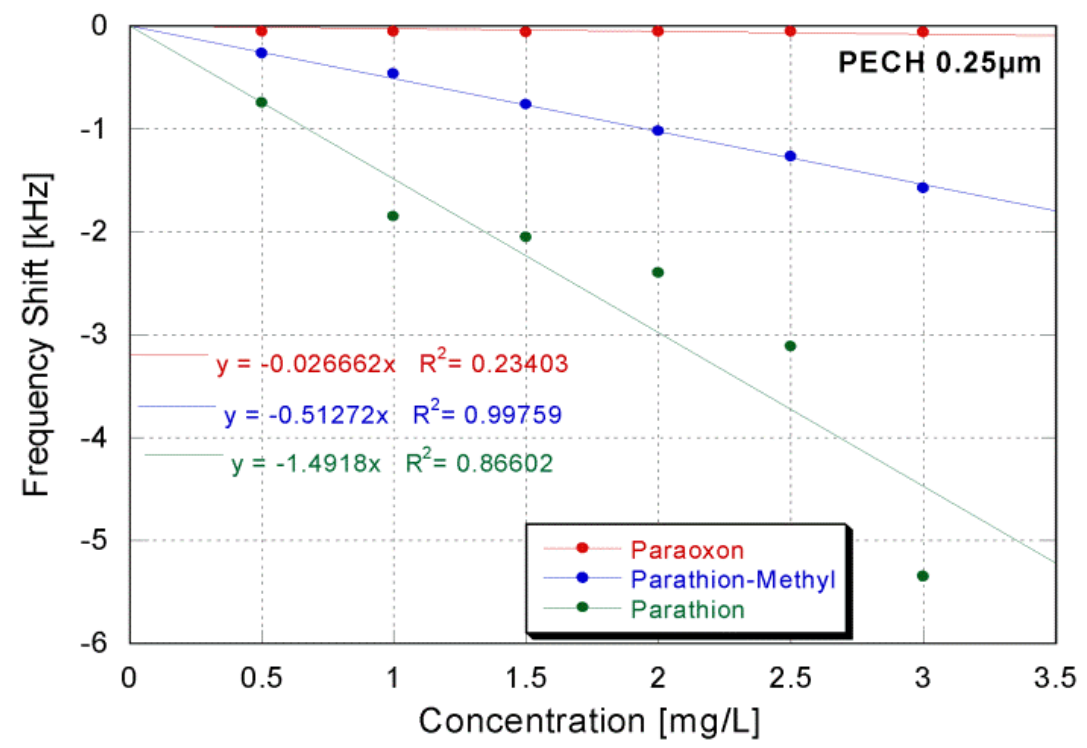

Figure 4.21: Sensitivity curve for the detection of $0.5 \mathrm{mg} / \mathrm{L}$ to $3.0 \mathrm{mg} / \mathrm{L}$ of parathion, parathionmethyl, and paraoxon using a guided SH-SAW device coated with $0.25 \mu \mathrm{m}$ of PECH.

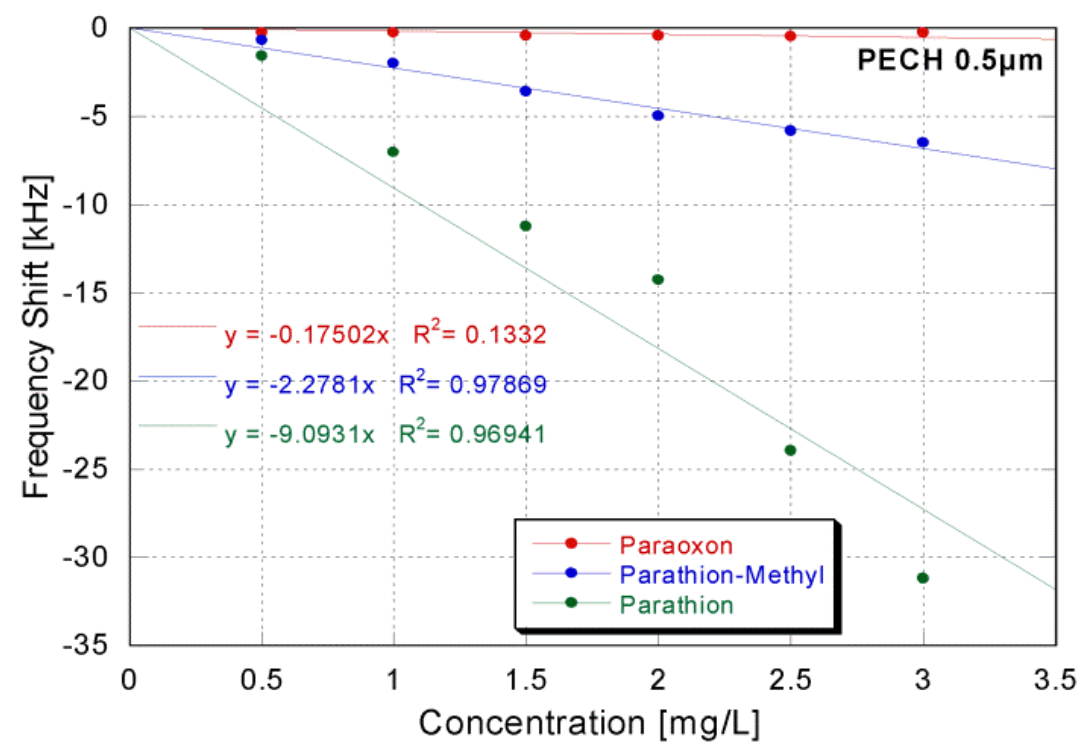

Figure 4.22: Sensitivity curve for the detection of $0.5 \mathrm{mg} / \mathrm{L}$ to $3.0 \mathrm{mg} / \mathrm{L}$ of parathion, parathionmethyl, and paraoxon using a guided SH-SAW device coated with $0.5 \mu \mathrm{m}$ of PECH. 


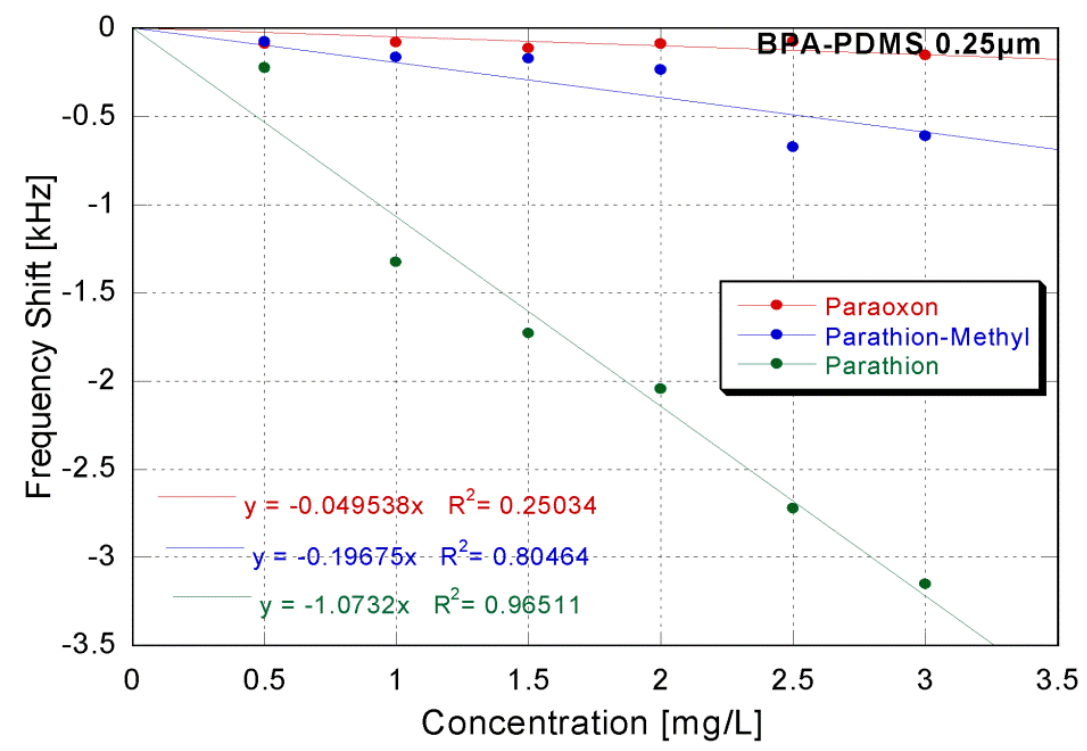

Figure 4.23: Sensitivity curve for the detection of $0.5 \mathrm{mg} / \mathrm{L}$ to $3.0 \mathrm{mg} / \mathrm{L}$ of parathion, parathionmethyl, and paraoxon using a guided SH-SAW device coated with $0.25 \mu \mathrm{m}$ of BPA-PDMS.

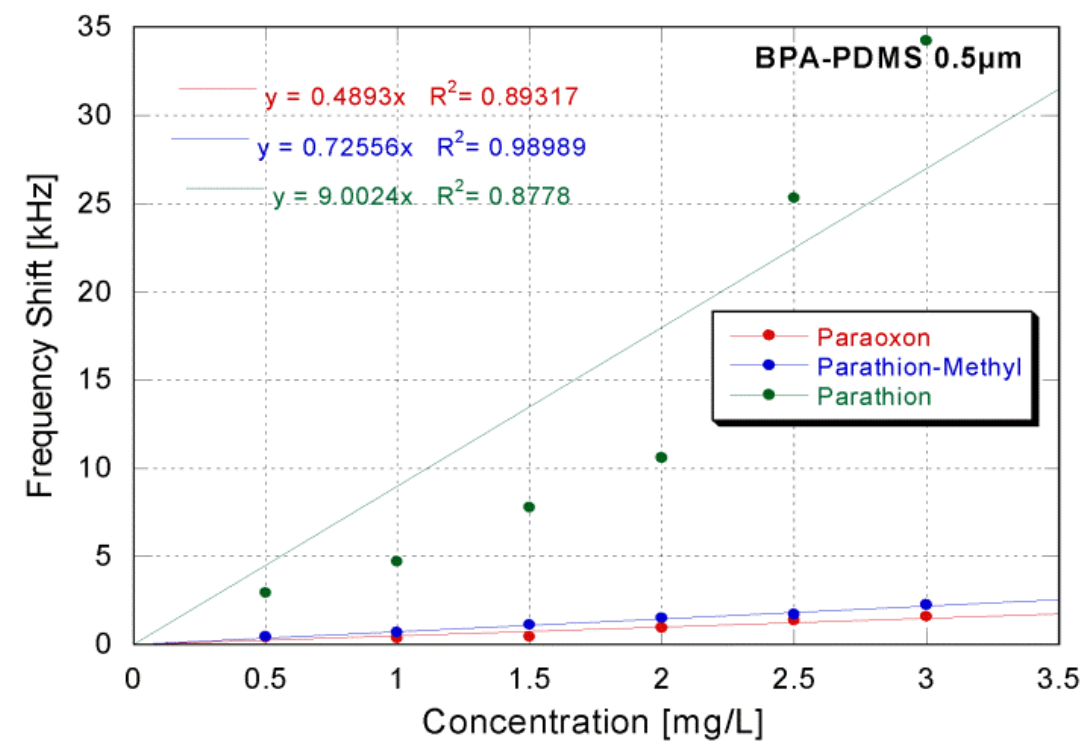

Figure 4.24: Sensitivity curve for the detection of $0.5 \mathrm{mg} / \mathrm{L}$ to $3.0 \mathrm{mg} / \mathrm{L}$ of parathion, parathionmethyl, and paraoxon using a guided SH-SAW device coated with $0.5 \mu \mathrm{m}$ of BPA-PDMS. 
The sensitivity was normalized with respect to the solubility of each analyte in water, and the results are shown in Figure 4.25 and tabulated in Table A.2 in the Appendix. Normalization can be done using the following equation

$$
\frac{\Delta f / M_{w}}{\left(\Delta C / C_{s}\right)}=\frac{\Delta f}{\Delta C} \times \frac{C_{s}}{M_{w}}
$$

where $\Delta f$ is the frequency shift, $\Delta C$ is the change in concentration, $C_{s}$ is the analyte solubility, and $M_{w}$ is the analyte molecular weight. The solubilities of parathion, paraoxon, and parathion-methyl in water are $12.9 \mathrm{mg} / \mathrm{L}, 2400 \mathrm{mg} / \mathrm{L}$, and $38 \mathrm{mg} / \mathrm{L}$, respectively $[39,47]$. The analytes are similar in molecular weight and therefore molecular weight was neglected in the normalization. The partitioning of an analyte between the aqueous and polymer phases is driven by the respective solubilities of the analyte in the two phases [48]. Assuming two analytes have similar solubilities in a given polymer, the analyte with the lower solubility in the aqueous phase will show higher enrichment in this polymer. Since paraoxon has the highest solubility in water, the (nonnormalized) sensitivity of the polymer towards the analyte will be the lowest. Parathion has the lowest solubility in water, explaining the observed results that the polymers show the highest sensitivity towards parathion in liquid. 


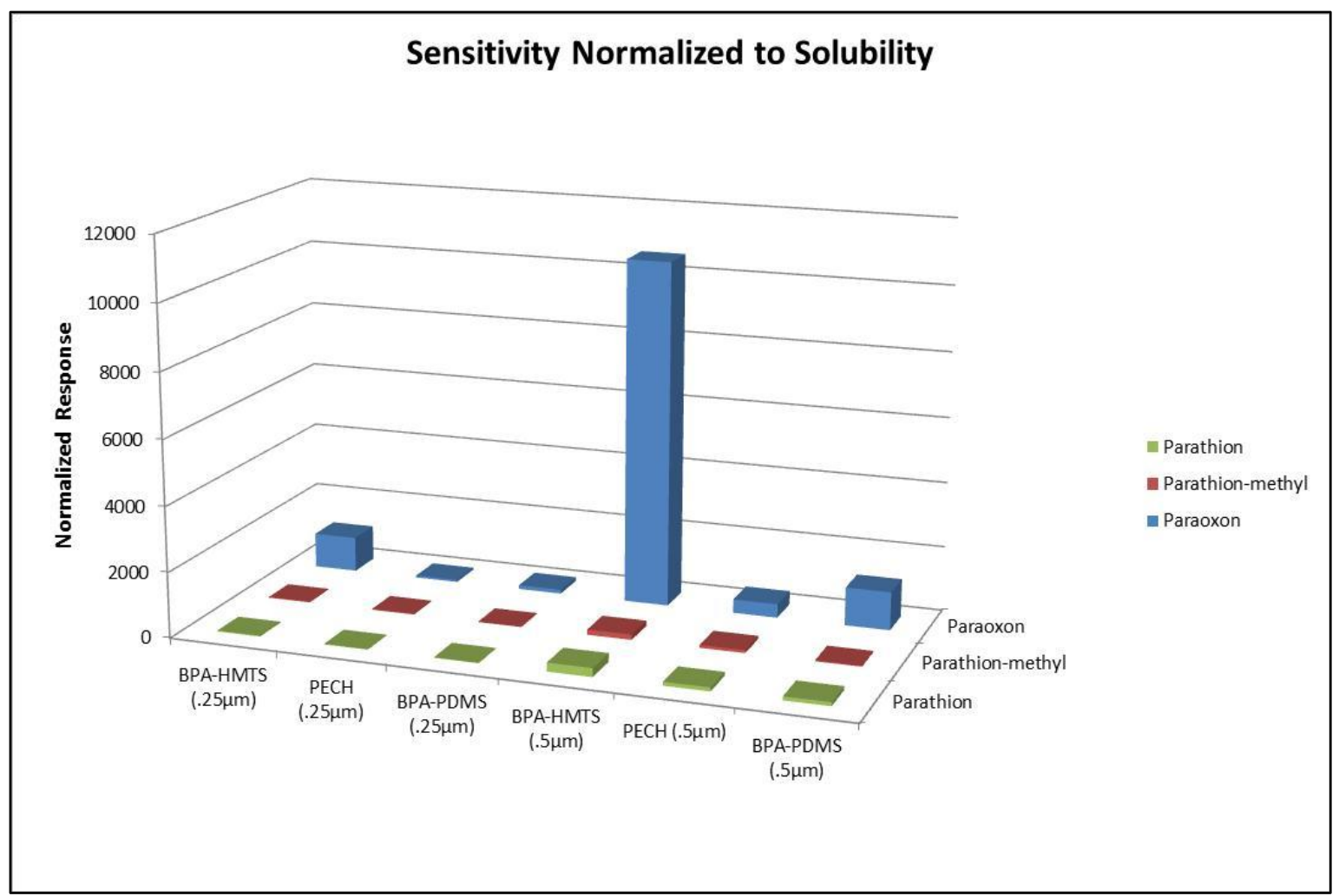

Figure 4.25: A comparison of the sensitivity of the polymers to the three analytes normalized to their solubility in water.

\subsection{Polymer Viscoelasticity Effect}

When polymers are spin coated onto a SH-SAW device, it is described as being rigidly bonded to the surface of the substrate. This means that the layer of polymer attached to the surface is moving synchronously with the substrate while the layer exposed to the air or liquid may lag with a specific phase shift $(\varphi)$. This can be broken down into three cases [22]. The first case occurs when the thickness of the film is thin enough ( i.e. $\varphi \ll \frac{\pi}{2}$ ) that the entire polymer layer moves synchronously with the substrate surface (case of an acoustically thin layer) [22]. In this thin film region, the fractional velocity and loss increase linearly with increasing polymer thickness. As the polymer thickness increases (i.e. $\varphi \rightarrow \frac{\pi}{2}$ ), the top surface layer of the polymer moves out of phase 
with the bottom layer (from this point on, the polymer will be referred to as acoustically thick). The fractional velocity change and loss begin to increase more rapidly with increasing polymer thickness. When $\varphi=\frac{\pi}{2}$, the polymer is at resonance due to constructive interference of the wave of the bottom of the polymer layer and the reflected wave from the top of the polymer layer [21]. For $\varphi>\frac{\pi}{2}$, the wave from the bottom layer of the polymer will destructively interfere with the wave reflected from the top surface of the film.

It can be seen that, when comparing the results for $0.5 \mu \mathrm{m}$-thick BPA-PDMS to those of other polymers, the frequency shift is in the opposite direction. These trends can be explained by the polymer viscoelasticity effect [26]. The effects of viscoelastic loading of a polymer depend on whether it is classified as a viscous or elastic polymer. The polymer viscoelastic effect was simulated, using the theory developed in Ref. 26, by varying values of $G^{\prime}$, the shear storage modulus representing elastic behavior and $G^{\prime \prime}$, the shear loss modulus representing viscous behavior. It is noted that the shear modulus, $G$, of the polymer, is given by the relationship $G=G^{\prime}+j G^{\prime \prime}$. The polymer is classified as a glassy film when $G^{\prime} \approx 10^{9} \mathrm{~Pa}$ and $G^{\prime \prime} \ll G^{\prime}$ or a rubbery film if $G^{\prime} \leq 10^{7} \mathrm{~Pa}$ and $G^{\prime \prime}$ is comparable or $<G^{\prime}[26]$. The sensor response is shown as a function of film thickness in Figure 4.26 .

The response of the $0.5 \mu \mathrm{m}$-thick BPA-PDMS coated device can be explained using Figure 4.26 (a) and (b). For this case, $G^{\prime \prime}=0.1 \mathrm{GPa}$ and $G^{\prime}$ varies from 0.01GPa (rubbery), 0.10GPa (intermediate), to 1.00GPa (glassy). For polymers at lower thicknesses, the viscoelastic changes do not have much of an effect on the sensor response. As the polymer is exposed to the analyte, it can be observed that the thickness 
slightly increases resulting in a minor increase of the magnitude of the change in velocity and the viscoelastic changes has more of a predominant effect [49]. When the thickness of the polymer approaches $0.5 \mu \mathrm{m}$, depending on the value of $G^{\prime}$, the change in velocity reaches a resonance point that correlates to inflection point of the loss. When the polymer thickness is greater than $0.5 \mu \mathrm{m}$, the magnitude of the change in velocity will decrease. These findings can qualitatively explain the results observed for BPA-PDMS, which largely behaves like an acoustically thin coating at lower thickness of $0.25 \mu \mathrm{m}$ but must be considered acoustically thick at a thickness of $0.5 \mu \mathrm{m}$. Note that in Figure 4.26 (a) the (negative) slope increases with increasing polymer thickness before the minimum in the change of velocity is reached. While the figure shows that the polymer doubles in thickness from $0.25 \mu \mathrm{m}$ to $0.5 \mu \mathrm{m}$, the gain in sensitivity can be larger than a factor of 2 . This effect was also observed with the experimental data; see Table A.1 in the Appendix.

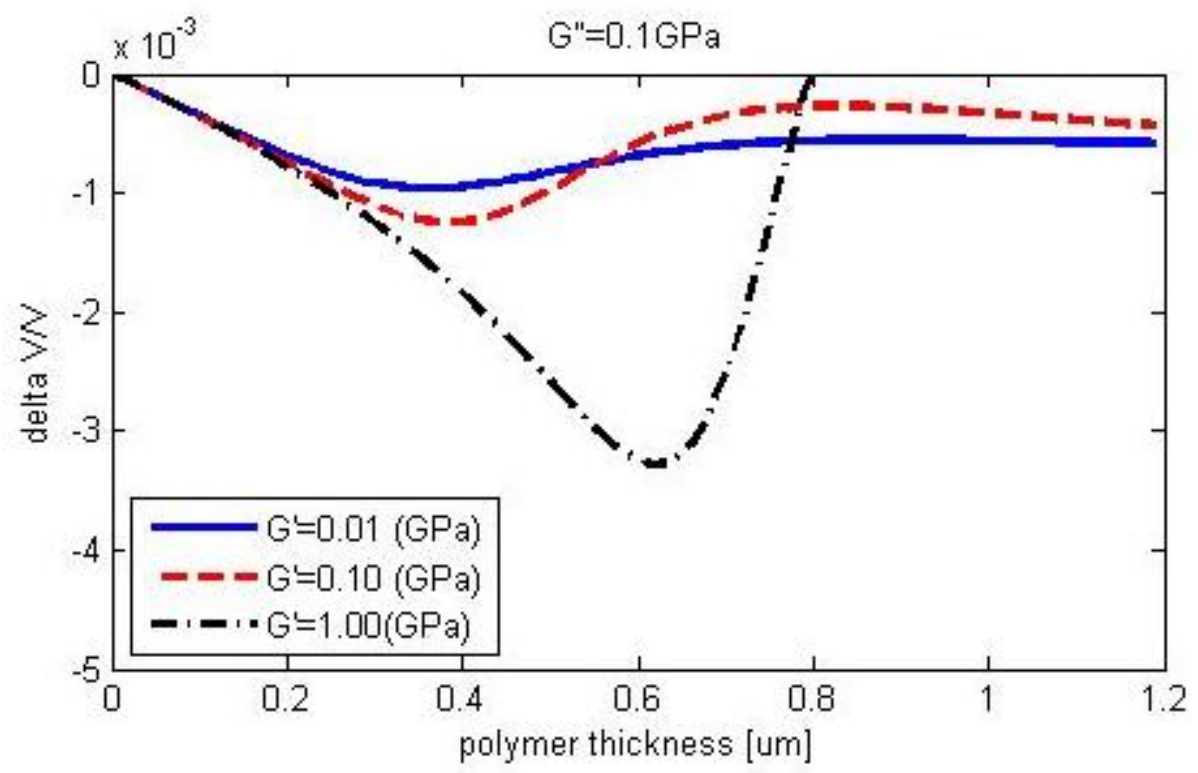

Figure 4.26 (a): Calculated fractional velocity change as a function of polymer thickness with $G^{\prime}$ as a parameter. 


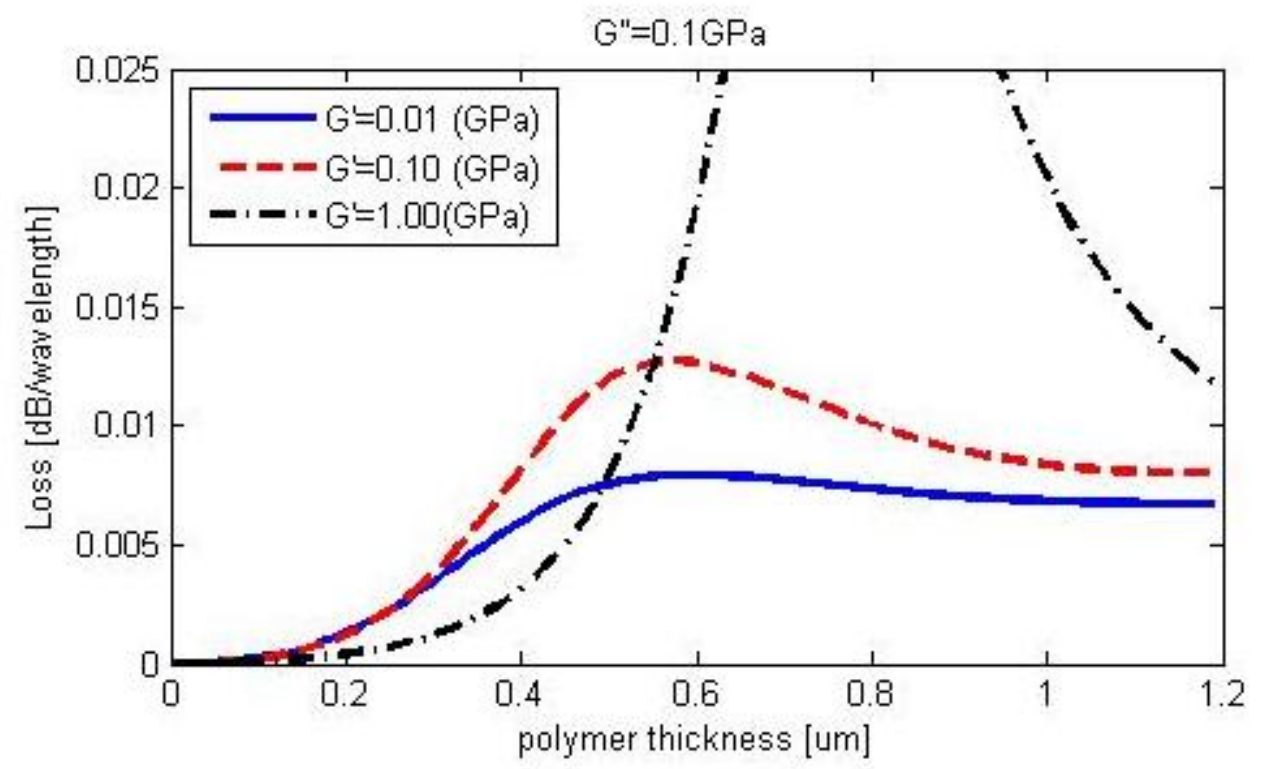

Figure 4.26 (b): Calculated loss response as a function of polymer thickness with G' as a parameter.

\subsection{Sensor Array Design}

The most important qualities of a sensor system are its sensitivity and selectivity.

Creating an array can help a sensor system to be more selective, thus allowing the investigation of mixtures of analytes or the identification of a single analyte. Once the response data is gathered, it can be processed to extract analyte-specific features or patterns, serving as a fingerprint which is specific to a target analyte. These patterns can be recognized using pattern recognition techniques [45].

A sensor array can be designed by assembling discrete coated devices or by combining several devices on one wafer. In order to design a sensor array, there is a need for several coatings. In the present case, the array is assembled using discrete devices with only a limited number of coatings available. To increase the number of coated 
devices in the array, an investigation on different coatings needs to be performed for thickness, selectivity, and sensitivity. The coatings used in the implementation of the sensor array for this work are presented in Table 4.1. This will result in a total of six devices in the array.

\begin{tabular}{|c|c|c|}
\hline BPA-HMTS & BPA-PDMS & PECH \\
\hline $\mathrm{h}_{1}=0.25 \mu \mathrm{m}$ & $\mathrm{h}_{1}=0.25 \mu \mathrm{m}$ & $\mathrm{h}_{1}=0.25 \mu \mathrm{m}$ \\
$\mathrm{h}_{2}=0.5 \mu \mathrm{m}$ & $\mathrm{h}_{2}=0.5 \mu \mathrm{m}$ & $\mathrm{h}_{2}=0.5 \mu \mathrm{m}$ \\
\hline
\end{tabular}

Table 4.1: The sensor array design using BPA-HMTS, BPA-PDMS, and PECH coated devices at a thickness of $0.25 \mu \mathrm{m}$ and $0.5 \mu \mathrm{m}$.

The sensor array can be implemented using a combination of any of the three polymers and the two thicknesses as long at their sensitivities provide independent information. This can lead up to a six-sensor device array if the coatings are all used. However, the response time can be added as an additional input parameter for pattern recognition provided that the response times are independent of the other input parameters. In this case, the array can be created from a combination of the frequency shifts and the response times of each coating/analyte pair. Figures 4.27-4.29 show a visual pattern recognition technique using radial plots for the analytes, parathion, paraoxon, and parathion-methyl, at concentrations from $0.5 \mathrm{mg} / \mathrm{L}$ to $3.0 \mathrm{mg} / \mathrm{L}$. These specific radial plots were made using the frequency shifts and time responses of BPAHMTS, BPA-PDMS, and PECH at a thickness of $0.5 \mu \mathrm{m}$ which can be found in Tables A.3-A.5 in the Appendix. The frequency shifts and time responses are normalized to their largest response in order for the radial plots to be on a comparative scale. A coating thickness of $0.5 \mu \mathrm{m}$ was chosen because of the larger sensitivity at this thickness; at this 
thickness, the coated devices will provide more reproducible and distinct response patterns for the different analytes.

When evaluating the radial plots, it can be seen from comparing Figs. 4.27(a-f), Figs. 4.28(a-f), and Figs. 4.29(a-f) that there is an obvious and distinct visual pattern for each analyte. The patterns are consistent throughout all of the concentrations in their respective analyte group. Because of the small frequency shifts at low concentrations, minor fluctuations are observed in the patterns. Uncertainty can be eliminated by evaluating another array with the same or different input parameters. At this time, single analyte identification is possible. For example, the criterion for detection of paraoxon could be that, for all concentrations investigated, the pattern is in the distinct shape of an arrow orientated towards the time response axis of the $0.5 \mu \mathrm{m}$-thick BPA-HMTS. 

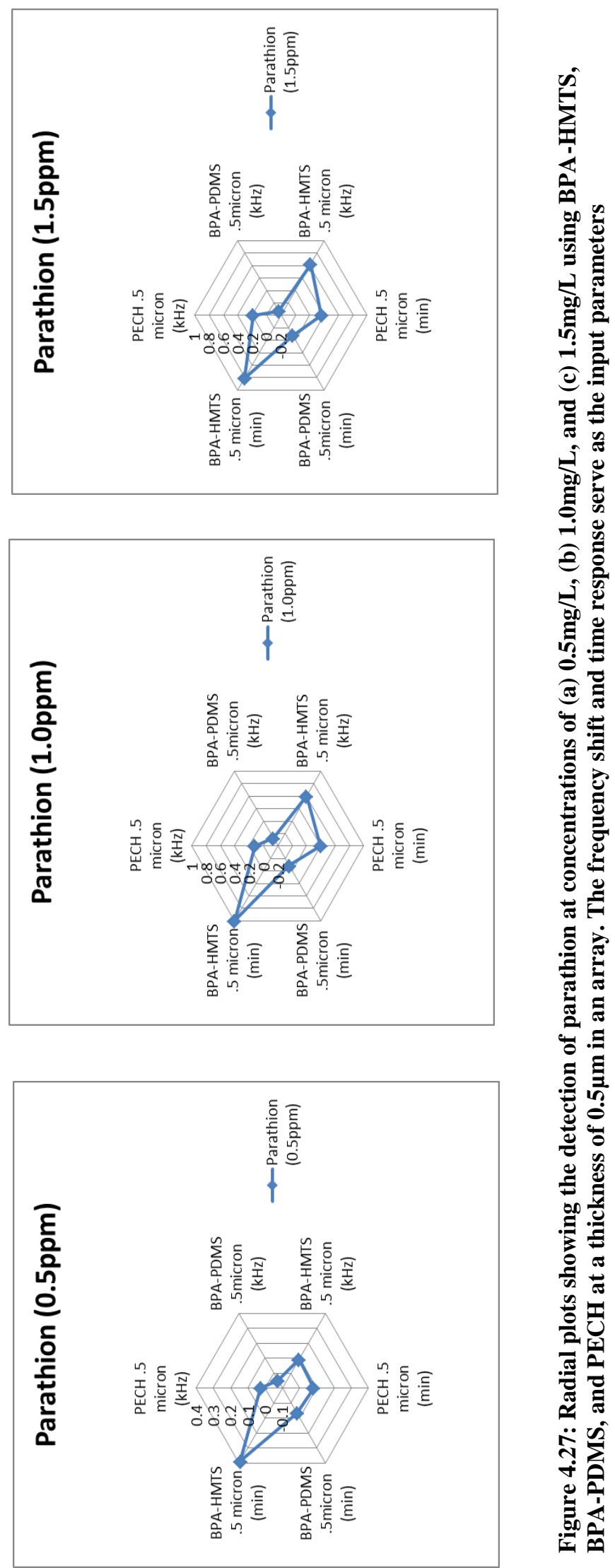

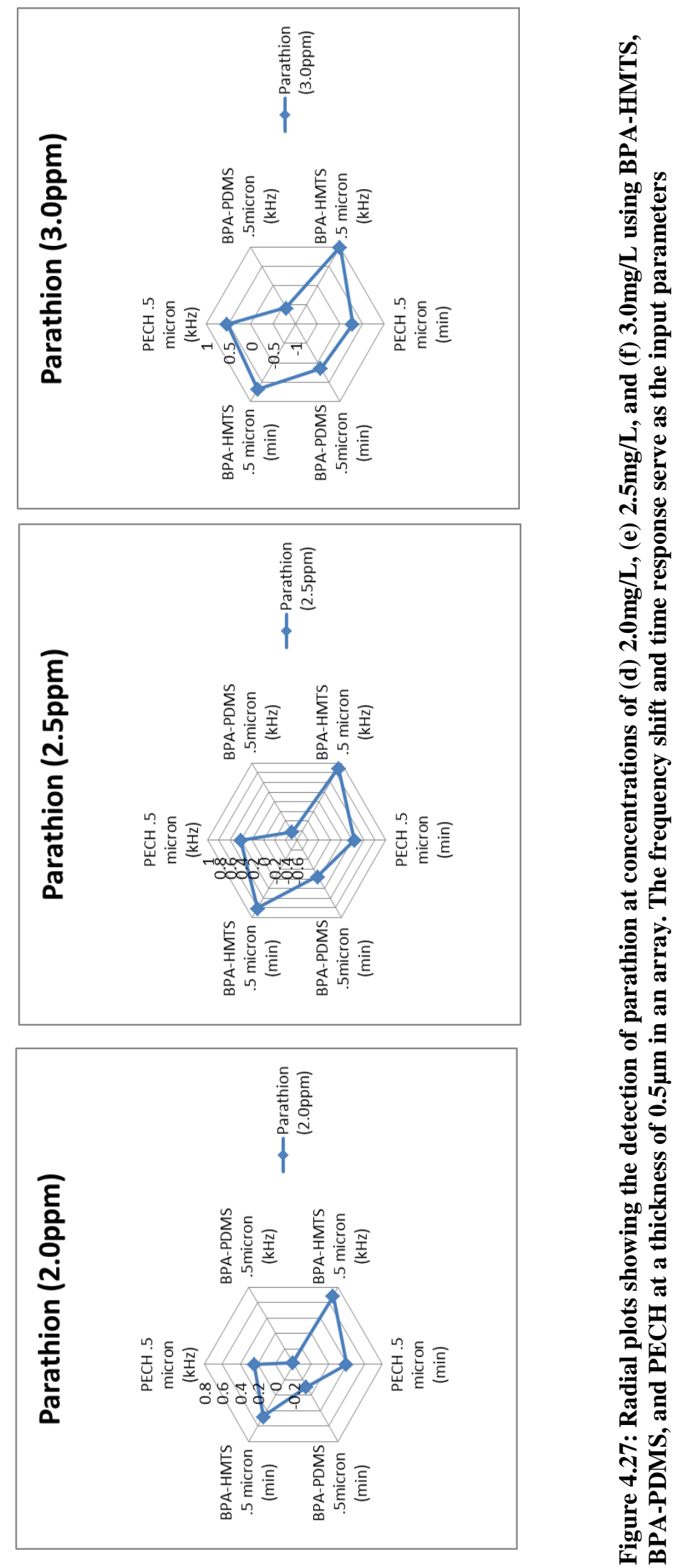

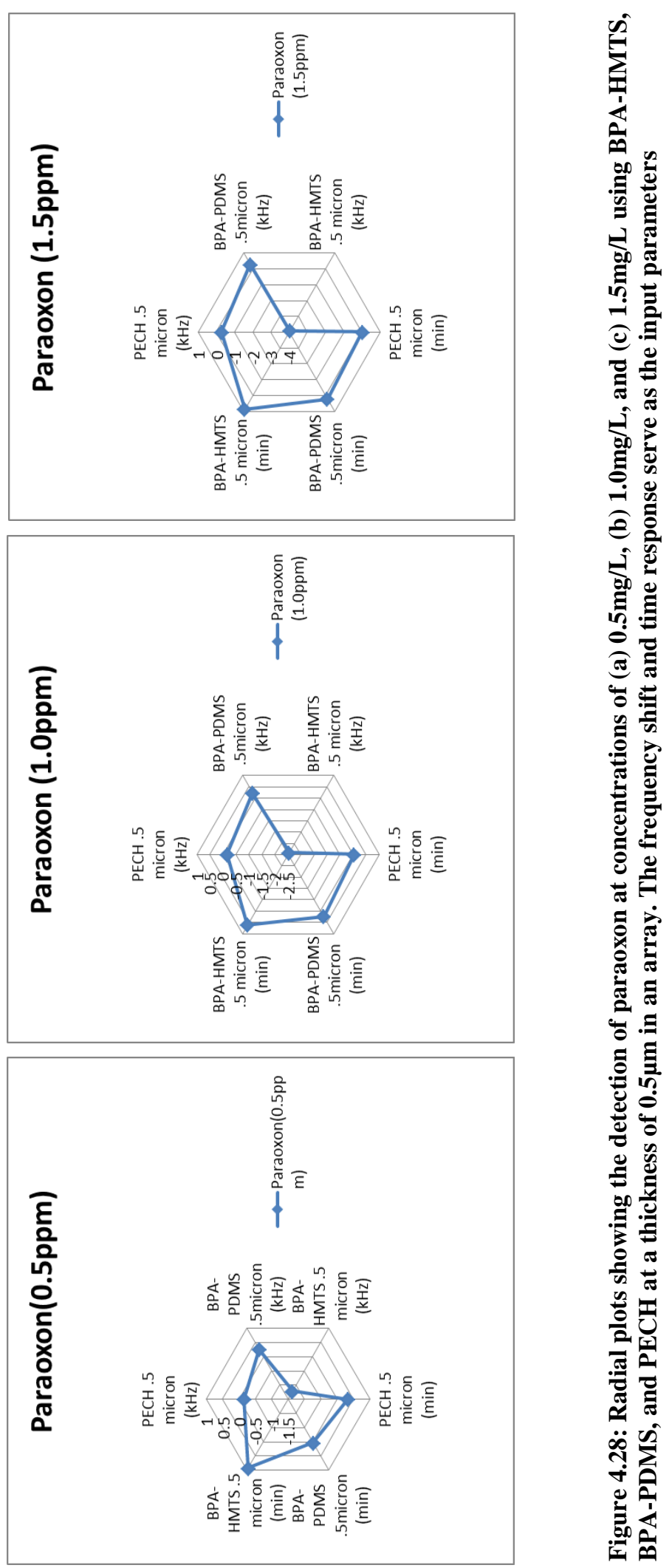

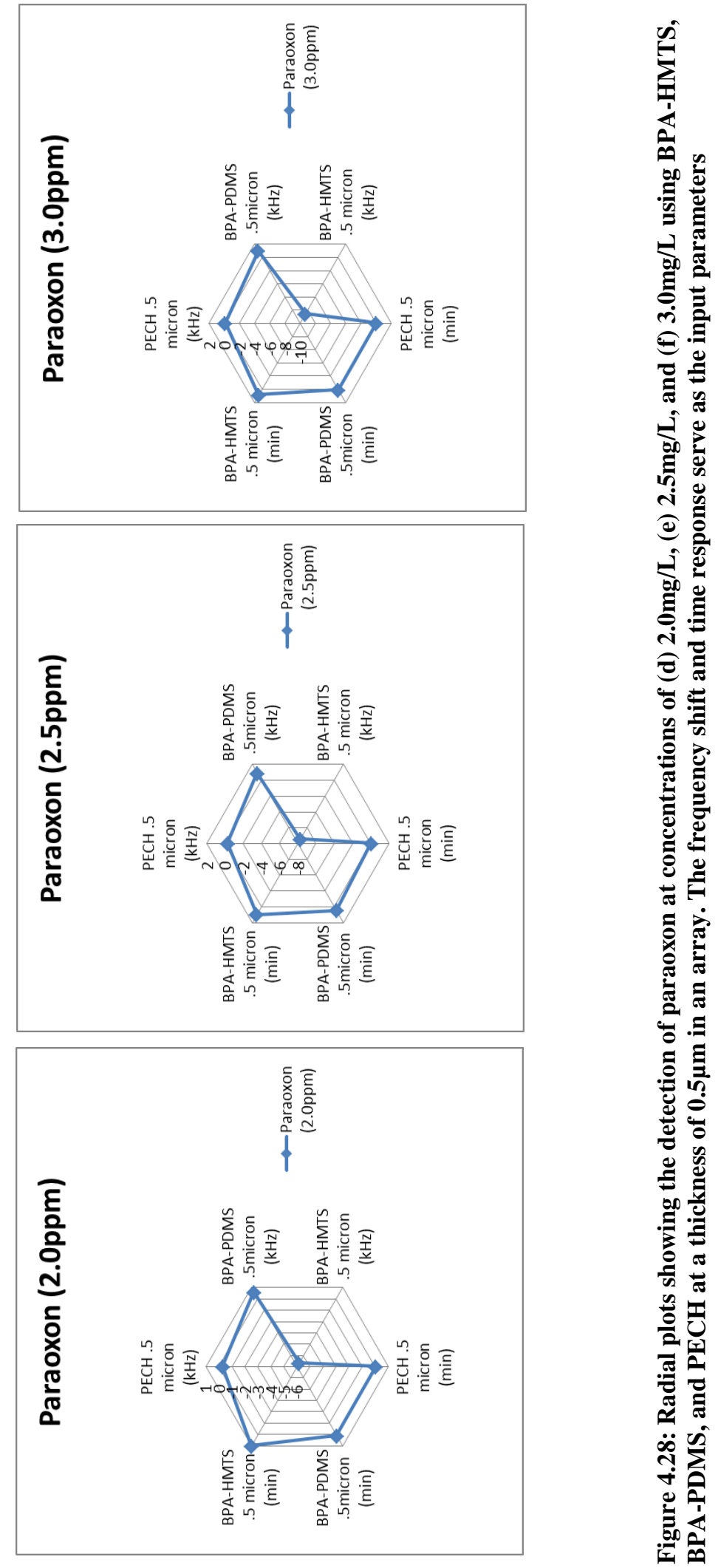

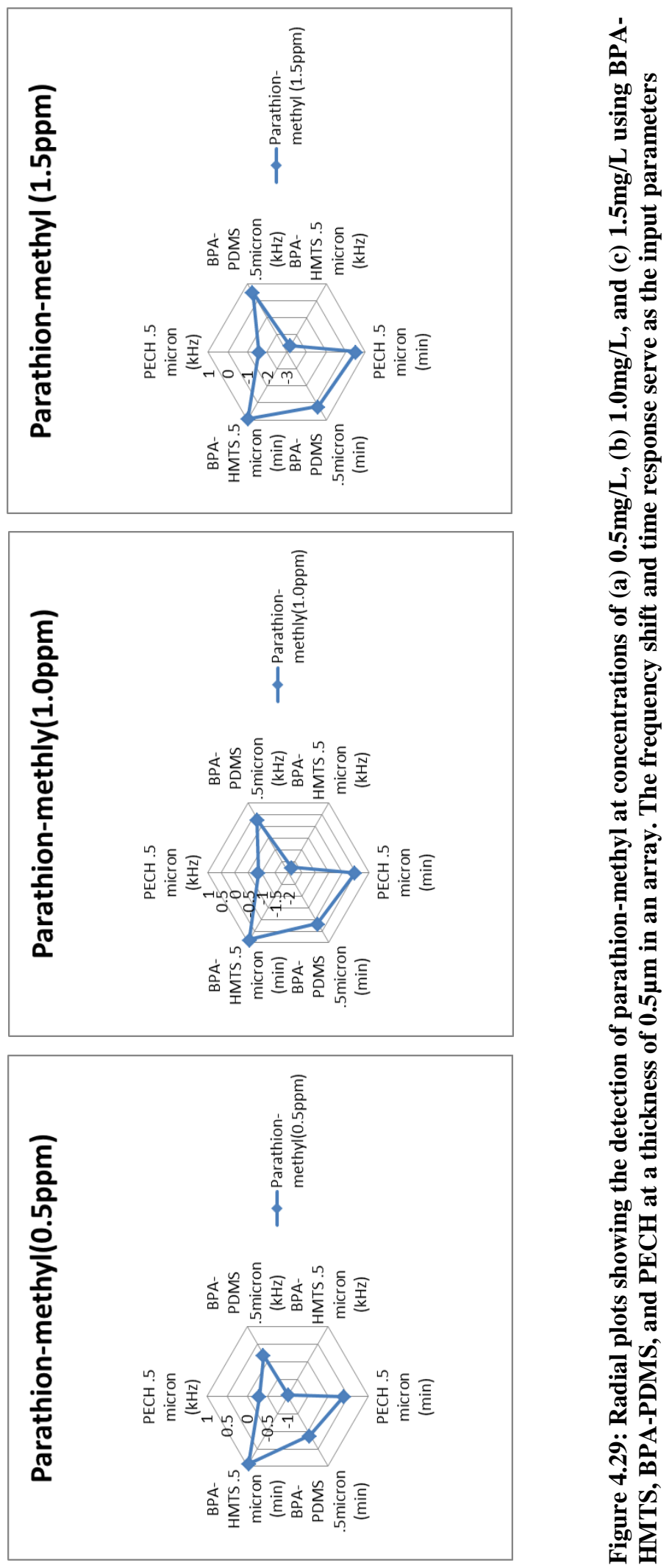

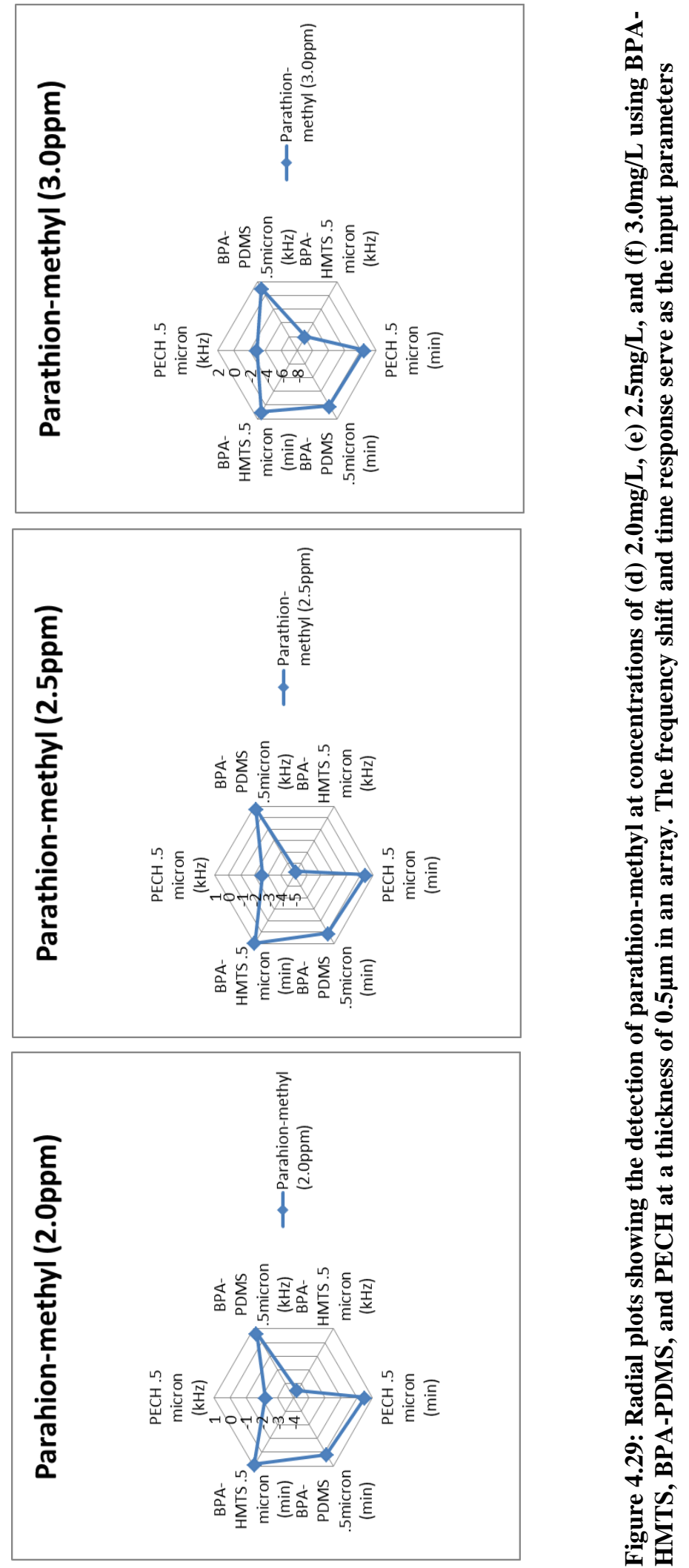
Once the analyte has been determined, the concentration can be quantified using an algorithm that computes the unknown sample's distance from the points in the known pattern in the three-dimensional space of the steady-state frequency shifts of the three $0.5 \mu \mathrm{m}$-thick polymer coatings [44]. The distance between the two points $y_{i}$ and $z_{i}$ can be calculated using

$$
\mathrm{d}(\overline{\mathrm{y}}, \overline{\mathrm{z}})=\left[\sum_{j=1}^{3}\left(y_{j}-z_{j}\right)^{2}\right]^{1 / 2}
$$

The unknown sample should exemplify a pattern that is identical to one of the known patterns; therefore, the distance between the two points should be minimal. The certainty of the unknown pattern being identical to the known pattern can be determined by a level of confidence [44]

$$
C_{i}=\frac{1}{d\left(\bar{y}, \overline{x_{i}}\right)}
$$

where $\overline{x_{i}}$ is a vector representing the known pattern. The level of confidence can be normalized and defined between the values of 0 and 1 , corresponding to least certain to most certain, respectively. Figs. 4.30-4.32 show the level of confidence for three different measured test samples. For Figs. 4.30 and 4.31, it is apparent that the level of confidence indicates measured test sample 1 to be $3.0 \mathrm{mg} / \mathrm{L}$ parathion and test sample 2 to be 2.0mg/L of paraoxon, respectively, which confirms the actual sample concentrations. Fig. 4.32, however, has two high levels of confidence $C_{i}=1$ corresponding to $0.5 \mathrm{mg} / \mathrm{L}$ of parathion-methyl and $C_{i}=.74$ corresponding to $0.5 \mathrm{mg} / \mathrm{L}$ of paraoxon. The higher level of confidence indicates that the measured test sample is $0.5 \mathrm{mg} / \mathrm{L}$ of parathion-methyl which, in fact, is the actual concentration. Figs. 4.33 and 4.34 shows the level of confidence for two different samples which lie exactly on the fitted line of the sensitivity 
curve, but whose concentrations were chosen not to match any of the measured concentration values. In Figure 4.33, the certainty of the sample being $1.5 \mathrm{mg} / \mathrm{L}$ of parathion is significantly higher when compared to the other analytes, which is in agreement with the chosen fitted value of $1.49 \mathrm{mg} / \mathrm{L}$ of parathion. In Fig. 4.34, the level of confidence curve shows a level line between $1.5 \mathrm{mg} / \mathrm{L}$ and $2.0 \mathrm{mg} / \mathrm{L}$ where the values of $c_{i}$ are equal. This type of curve is indicating that the actual concentration should be close to $1.75 \mathrm{mg} / \mathrm{L}$ of parathion, which is the concentration of the chosen fitted value. This specific concentration of $1.75 \mathrm{mg} / \mathrm{L}$ was chosen to show that this algorithm can be successful for concentrations that were not obtained in the measured data set. In order to increase the accuracy of the determination of concentrations, more data points should be collected within the same range of $0.5 \mathrm{mg} / \mathrm{L}$ to $3.0 \mathrm{mg} / \mathrm{L}$ for each coating/analyte pair. It should be noted that there may be discrepancies for analyte identification between any small concentrations (for example $0.5 \mathrm{mg} / \mathrm{L}$ ) of the analyte when using the radial plots. In this case, the algorithm used to determine the level of confidence can be used as an extra tool for analyte identification. An advantage of the level-of-confidence method is that it quantifies the similarity of the response pattern of an unknown sample with known response patterns. On the other hand, an advantage of the radial plots used above is that it uses a total of six input parameters (three steady-state frequency shifts and three response times). 


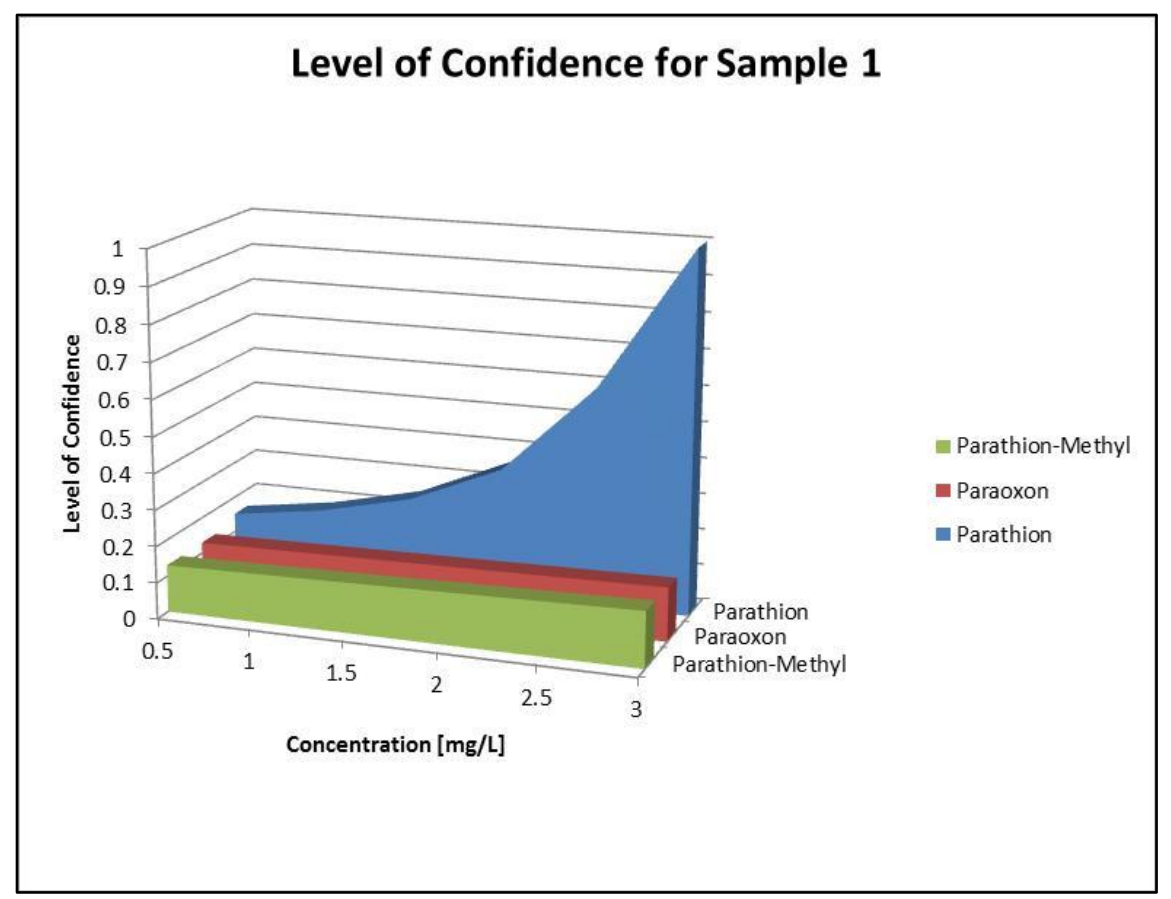

Figure 4.30: The level of confidence for test sample 1, points towards $3.0 \mathrm{mg} / \mathrm{L}$ of parathion as predicted by a pattern recognition technique based on an algorithm which computes the distance between the test sample and the known patterns.

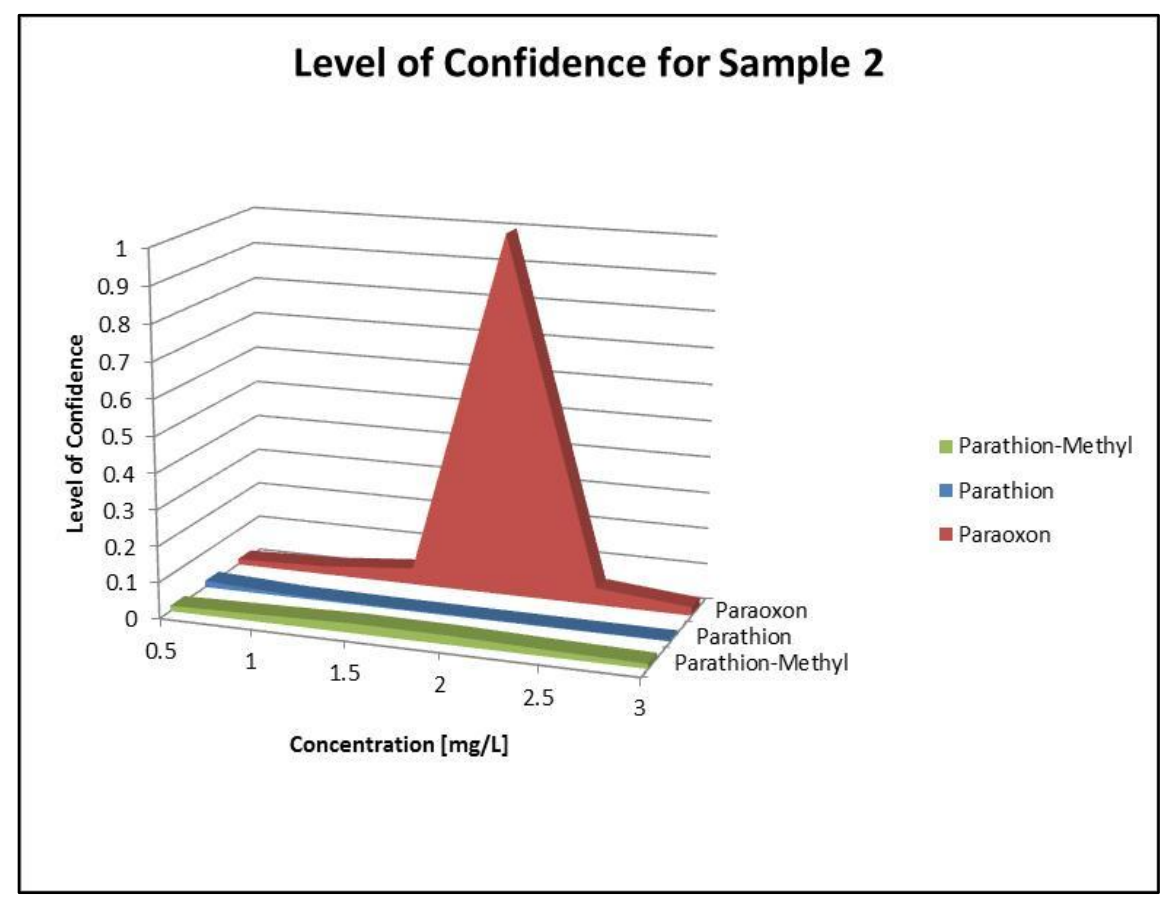

Figure 4.31: The level of confidence for test sample 3, points towards $2.0 \mathrm{mg} / \mathrm{L}$ of paraoxon as predicted by a pattern recognition technique based on an algorithm which computes the distance between the test sample and the known patterns. 


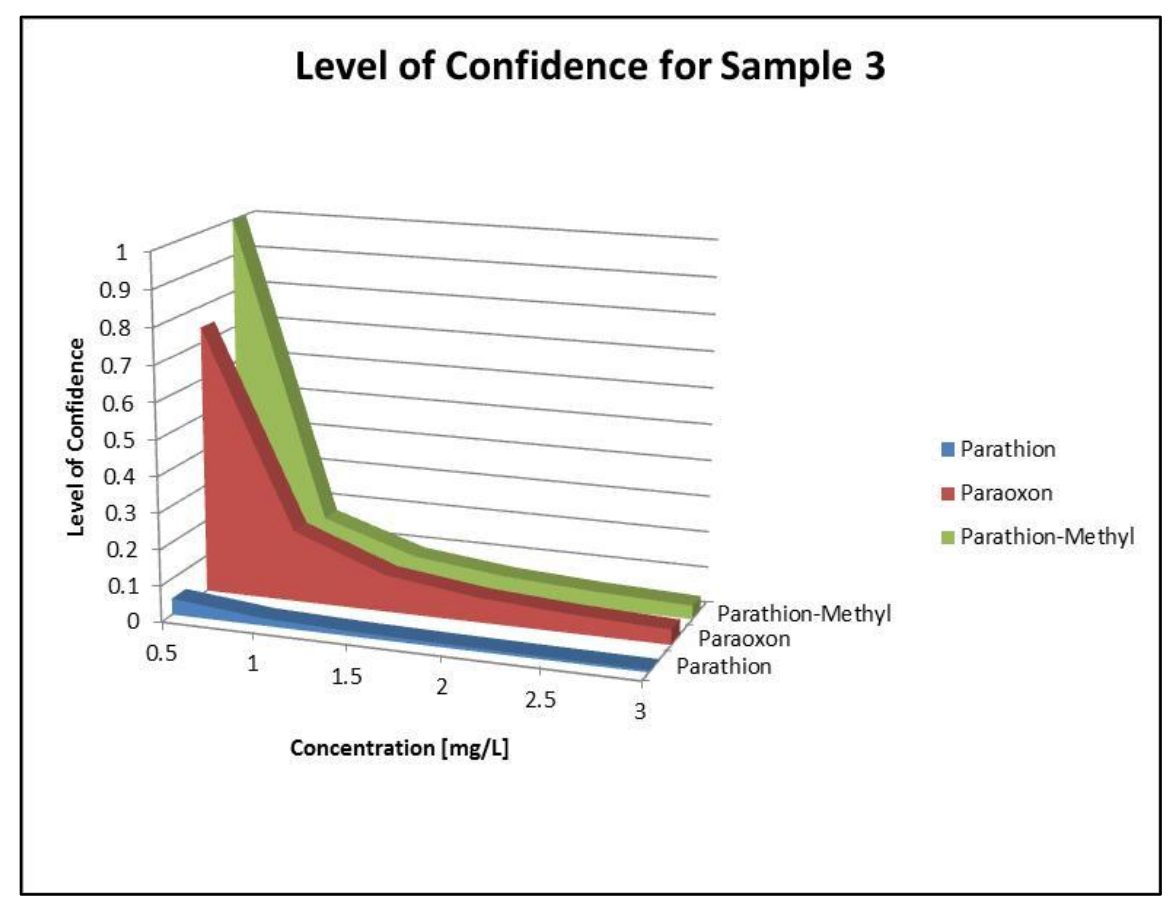

Figure 4.32: The level of confidence for test sample 3, points towards $0.5 \mathrm{mg} / \mathrm{L}$ of parathion-methyl as predicted by a pattern recognition technique based on an algorithm which computes the distance between the test sample and the known patterns.

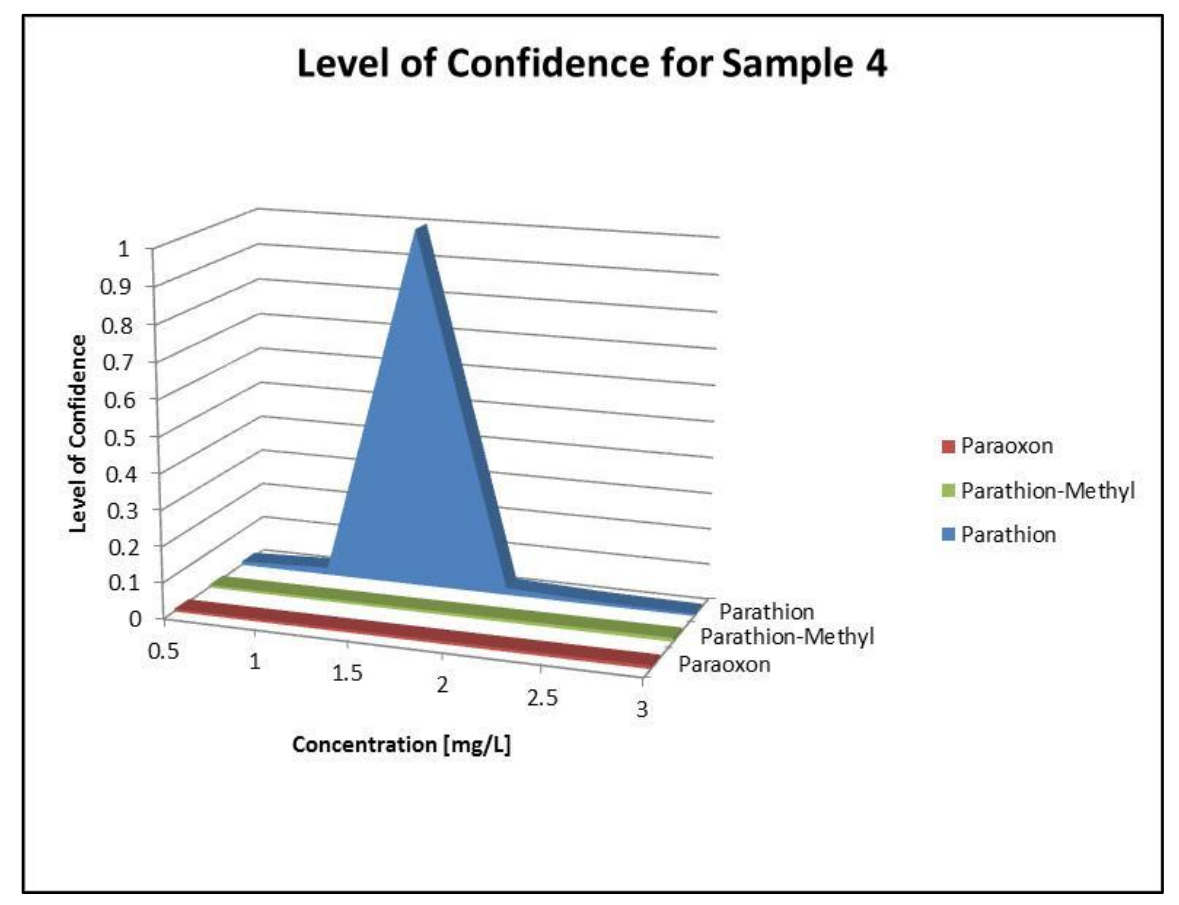

Figure 4.33: The level of confidence for test sample 4, points towards $1.5 \mathrm{mg} / \mathrm{L}$ of parathion as predicted by a pattern recognition technique based on an algorithm which computes the distance between the test sample and the known patterns. 


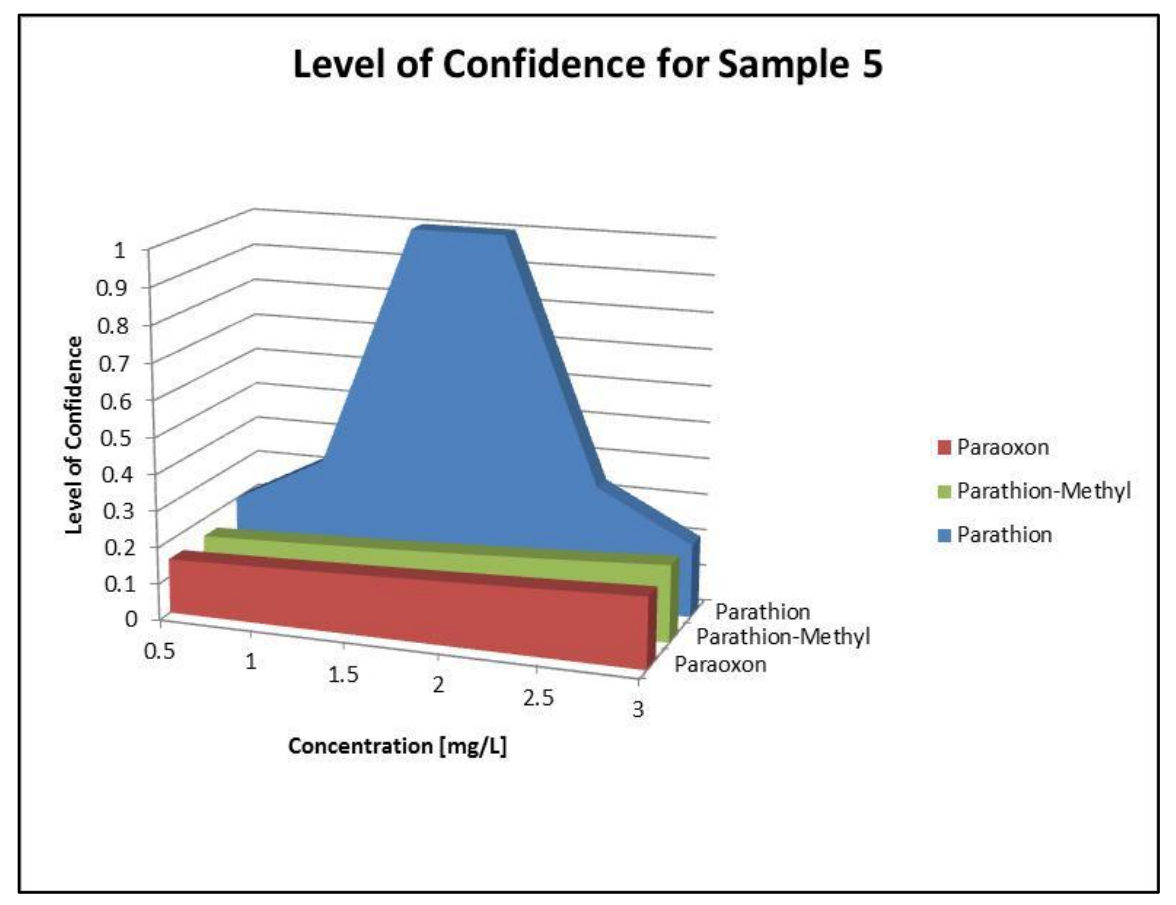

Figure 4.34: The level of confidence for test sample 5, points towards $1.75 \mathrm{mg} / \mathrm{L}$ of parathion as predicted by a pattern recognition technique based on an algorithm which computes the distance between the test sample and the known patterns.

\subsection{Practical Case Application}

In order to be able to use the sensor out in the field, it must maintain its stability, sensitivity, and reproducibility under the presence of interferents (physical and chemical contaminants found in groundwater). To simulate such a situation, the coated devices were tested for changes in the response as a function of $\mathrm{pH}$ and the presence of sediments. The responses to changes in the $\mathrm{pH}$ and presence of sediments are shown in Figure 4.35 and Figure 4.36, respectively. The experiments for the different $\mathrm{pH}$ levels (6.2, 7.0 and 8.0) were performed under otherwise identical conditions. The same also applies to the experiments with red clay. It should be noted that the sensor was placed face down during the experiments with the red clay to prevent precipitation on the sensor surface. The sensor proves to be unaffected to the presence of interferents. The 
discrepancies shown in Figure 4.36 for $2.5 \mathrm{mg} / \mathrm{L}$ and $3.0 \mathrm{mg} / \mathrm{L}$ of parathion-methyl may be due to measurement errors and/or concentration errors in the prepared solutions.

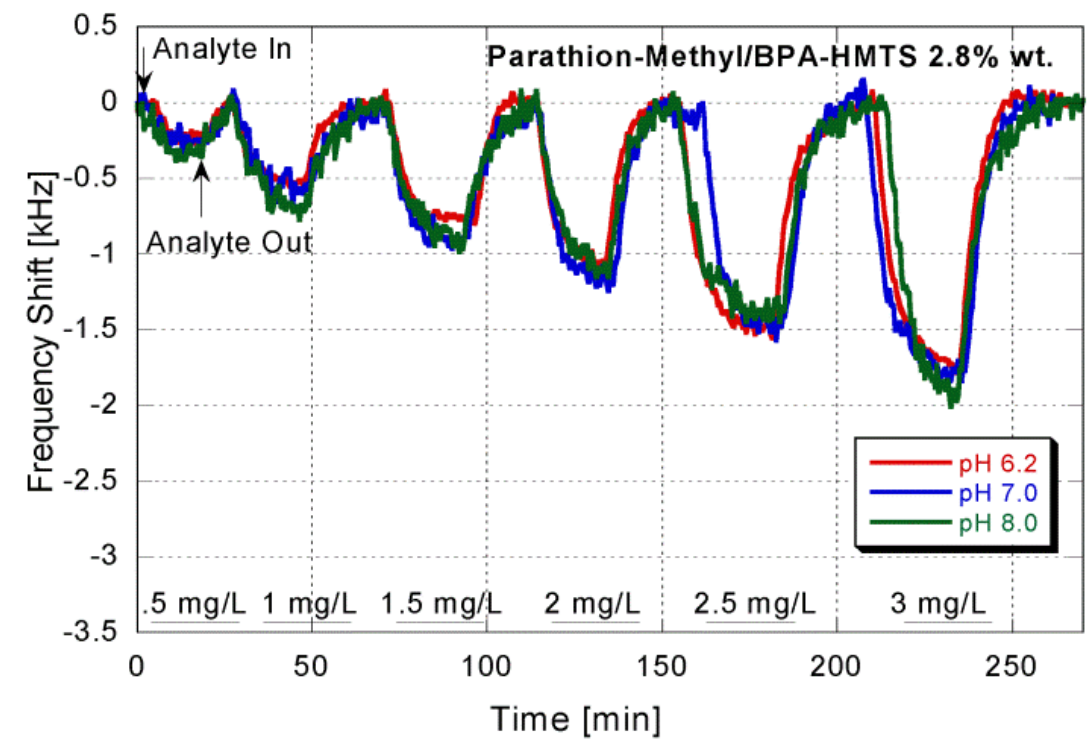

Figure 4.35: Comparing measured change in frequency shifts upon exposure of the BPA-HMTS $0.25 \mu \mathrm{m}$ coated device to $0.5 \mathrm{mg} / \mathrm{L}, 1.0 \mathrm{mg} / \mathrm{L}, 1.5 \mathrm{mg} / \mathrm{L}, 2.0 \mathrm{mg} / \mathrm{L}, 2.5 \mathrm{mg} / \mathrm{L}$, and $3.0 \mathrm{mg} / \mathrm{L}$ of parathionmethyl at pH levels of 6.2, 7.0, and 8.0.

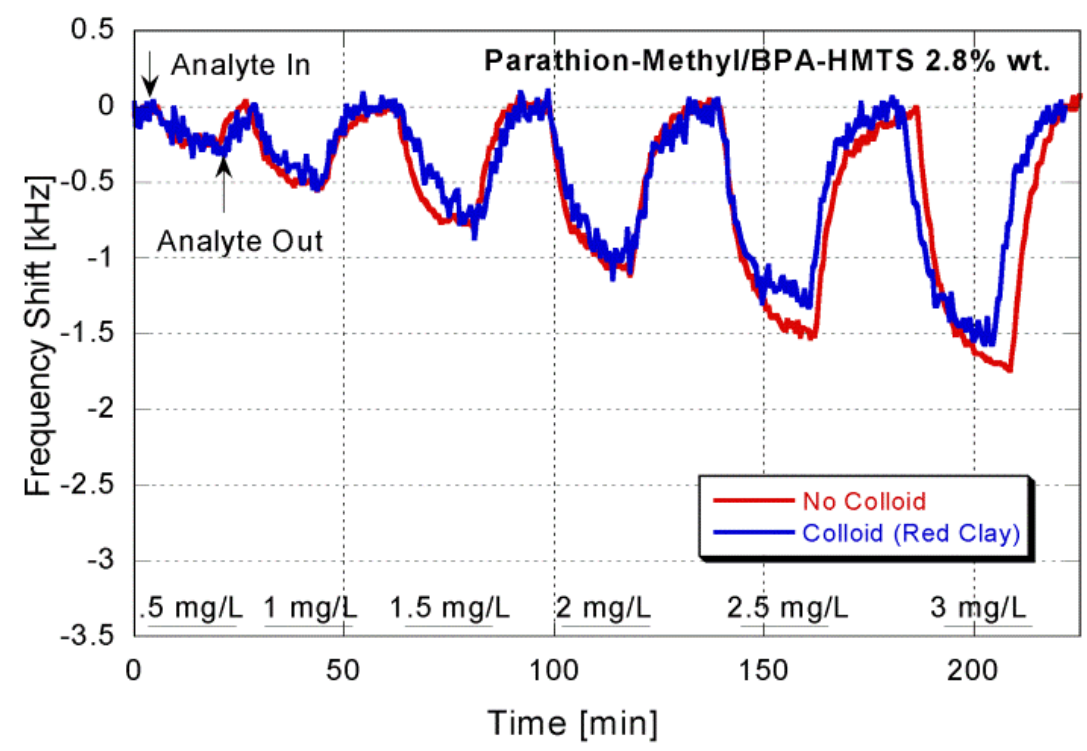

Figure 4.36: Comparing measured change in frequency shifts upon exposure of the BPA-HMTS $0.25 \mu \mathrm{m}$ coated device to $0.5 \mathrm{mg} / \mathrm{L}, 1.0 \mathrm{mg} / \mathrm{L}, 1.5 \mathrm{mg} / \mathrm{L}, 2.0 \mathrm{mg} / \mathrm{L}, 2.5 \mathrm{mg} / \mathrm{L}$, and $3.0 \mathrm{mg} / \mathrm{L}$ of parathionmethyl in the presence of red clay. 


\section{SUMMARY, CONCLUSIONS, AND FUTURE WORK}

\subsection{Summary}

Polymer coated SH-SAW sensors were investigated for the detection of organophosphates in aqueous solutions. The SH-SAW device utilizes the three-layer

geometry (liquid, polymer, and substrate) and three partially selective chemical layers to develop a highly sensitive platform for the detection of organophosphates. The sensor response was also modeled to extract the sensing parameters. Transient information (adsorption time response) and steady state response are extracted using a single exponential fit. These same coated sensors are used in a sensor array for analyte identification and concentration determination. The sensor will be characterized for its ruggedness, detection limits, and its selectivity. The end goal of the project will be the actual use of the sensors in the field.

A background on pesticides and its current use in the world were presented with a rationale for the monitoring and detection at contaminated sites. An overview of chemical sensors was presented with a focus on acoustic wave based sensors. The various types of acoustic wave- based sensors, including the SH-SAW sensor, were compared with a focus on those configurations that are suitable for liquid based applications.

The SH-SAW device was reviewed as liquid-phase sensing platform. A detailed description was given on the theory of the SH-SAW measured responses and how they are related to perturbations in wave propagation characteristics (wave velocity and attenuation). Several assumptions were made in order to simplify the modeling of the 
sensor response and identify the appropriate variables that can affect the sensor response. The polymer coating was then introduced in order to account for analyte sorption into the coating and its contribution to the sensor response. A previously developed model for the sorption process was used which includes the role of the shear modulus of a polymer in the sensor response [24].

Three partially chemically selective polymers (BPA-HMTS, BPA-PDMS, and $\mathrm{PECH})$ were investigated for their affinities to organophosphate analytes parathion, paraoxon, and parathion-methyl. The range of concentrations which was measured for each analyte was from $0.5 \mathrm{mg} / \mathrm{L}$ to $3.0 \mathrm{mg} / \mathrm{L}$. Each polymer was tested at a thickness of $0.25 \mu \mathrm{m}$ and $0.5 \mu \mathrm{m}$ to study the effects of polymer thickness on device performance (sensitivity, response time, etc.). The steady state frequency shift and time response were extracted from the measured frequency responses. An exponential fit was used to represent the obtained sensor data. The frequency sensitivities were calculated using the fitted slope of the frequency response versus concentration curve. The sensitivity of a coating to a given analyte was also normalized with respect to solubility in water for the three organophosphates.

The sensor was also tested for reproducibility, stability and sensitivity under real case applications. The devices were exposed and tested for different $\mathrm{pH}$ levels of 6.2, 7.0, and 8.0 to simulate the possibility of varying $\mathrm{pH}$ levels of groundwater. The devices were also tested with colloids to simulate the sensor's response when in contact with physical contaminants often present in groundwater.

An array of sensors was designed and implemented to increase selectivity through 
analyte identification and determination of concentration. The array data consist of both the steady state frequency shift and time responses for $0.5 \mu \mathrm{m}$-thick BPA-HMTS, BPAPDMS, and PECH. Analyte identification was determined using pattern recognition via radial plots with both steady state frequency shift and time responses as input parameters. Sample concentration was determined using an algorithm that computes the distance of the unknown sample to the pattern of the known collected samples in the threedimensional space of steady-state frequency responses. The level of confidence was calculated and plotted for the test samples, using the reciprocal of the distance.

\subsection{Conclusions}

In the present work, it was demonstrated that polymer (BPA-HMTS, BPA-PDMS, and PECH) coated SH-SAW devices can be used for the detection of organophosphates in a liquid environment. The sensor response (i.e. frequency shift) was shown to be a function of both mass loading and modulus change resulting from analyte sorption into the polymers. The insertion loss response was not shown due to insignificant sensor responses in the ranges of measured concentration. The $0.5 \mu \mathrm{m}$-thick polymer coated device also showed a greater sensitivity than the $0.25 \mu \mathrm{m}$-coated device, as a result of a more pronounced effect of mass loading and modulus change. The sensor response exhibited a high sensitivity for the polymers BPA-HMTS, BPA-PDMS, and PECH for parathion, parathion-methyl, and paraoxon, respectively, but with a varying degree of selectivity. It was noted that the higher the solubility of an analyte in water, the lower the partition coefficient of this analyte in a coating which therefore resulted in a smaller response for the coated device. Therefore, paraoxon, which has the highest solubility in water, produced the lowest sensitivity among the analytes for all three polymers. On the 
other hand, parathion with the lowest solubility produced the highest sensitivity. It was observed that the frequency response returned back to the baseline when the analyte was removed. Therefore, the analyte/polymer interaction was reversible. The measured steady-state frequency shifts show a linear behavior with analyte concentrations within the measured concentration range. The slight deviation from linearity of the response was probably due to errors in the prepared analyte solution concentrations. Measurements have been performed several times and the error is approximately $\pm 18 \%$. The error bar is not shown in the figures as a fair determination will also require error bars for the changes in concentration, which were not determined. The response times of BPAHMTS, BPA-PDMS, and PECH followed the trend from slowest to fastest to parathion, parathion-methyl, and paraoxon, respectively, with the longest response recorded at about 120 minutes. This was explained by the larger free volume of polymer allowing for more absorption of the analytes. Other effects include the glassy/rubbery state of the polymers, the pore size of the polymer and the analyte molecule dimension, and the rate of analyte transport. The response of a $0.5 \mu \mathrm{m}$-thick BPA-PDMS for all three analytes was found to be positive, thus indicating the properties of an acoustically thick polymer undergoing the polymer viscoelasticity effect. This effect occurs if a large phase lag exists between the top and bottom surfaces of the vibrating polymer. This can then lead to an increased sensitivity and even a change in the sign of the frequency shift observed during analyte sorption [26].

In order to increase sensor selectivity, a sensor array was designed using several discrete devices with three coatings at two thicknesses. The sensor array can provide a pattern specific to a certain analyte. A visual pattern recognition technique was designed 
using radial plots with the axes representing the frequency shifts and time responses for BPA-HMTS, BPA-PDMS, and PECH at a thickness of $0.5 \mu \mathrm{m}$. This successfully indicated three distinct patterns for each analyte throughout all concentrations. The thickness of $0.5 \mu \mathrm{m}$ was chosen due to the higher sensitivity at this thickness rather than that of the thickness of $0.25 \mu \mathrm{m}$. The frequency shift and response times were normalized to their largest response for comparison purposes. Minor differences in the patterns were observed and are due to fluctuations in the prepared concentration samples. The level of confidence was presented to quantify the similarity between known response patterns and unknown fitted response pattern. The results showed a high level of confidence for the measured test samples (i.e.3.0mg/L of parathion, $2.0 \mathrm{mg} / \mathrm{L}$ of paraoxon, $0.5 \mathrm{mg} / \mathrm{L}$ of parathion-methyl) and for the solution samples that were not included in the pattern recognition design database (i.e. $1.49 \mathrm{mg} / \mathrm{L}$ of parathion and $1.75 \mathrm{mg} / \mathrm{L}$ of parathion). It was noted that the highest level of confidence for test sample 5 was the same between $1.5 \mathrm{mg} / \mathrm{L}$ and $2.0 \mathrm{mg} / \mathrm{L}$, indicating the algorithm correctly identifies the test sample as $1.75 \mathrm{mg} / \mathrm{L}$ of parathion. In order to obtain a more accurate estimate of analyte concentration, more measured samples need to be collected, or more data points need to be interpolated between known response patterns.

The sensor was tested for stability, sensitivity, and reproducibility by observing its performance under real case applications. When the sensor was tested for groundwater at different $\mathrm{pH}$ levels of 6.2, 7.0, and 8.0, the sensor response proved to be unaffected. The results also remain unchanged when comparing the response of samples with and without colloids (red clay). The results indicate that neither $\mathrm{pH}$ level nor the presence of colloids significantly interferes with analyte detection. 


\subsection{Future Work}

The present status of this work indicates the need for further improvement. This includes further investigation and characterization of the polymers, BPA-HMTS and BPA-PDMS, to increase reproducibility and stability. The current synthesis procedure of BPA-PDMS and BPA-HMTS, which are synthesized at Marquette University, produces a polymer that yields an inconsistent polymer layer (non-uniform coating) and nonreproducible sensor response (inconsistent steady state frequency shifts). These polymers also lack long term stability. These problems are especially observed with BPA-PDMS. The synthesis procedure needs to be revised to offer a more consistent polymer with reproducible results. This should include an additional procedure for a polymer stock solution for long term storage instead of preparing a fresh polymer solution when it is needed.

In order to create a more efficient sensor array, the sensor platform and the sensor cell should be redesigned to include several delay lines on the same substrate. In theory, a greater number of delay lines results in a more selective sensor. Each delay line will be coated with a different chemically sensitive polymer. This will allow for the possibility of just one test to be run for the detection of a specific analyte in a multicomponent sample. Another way to increase efficiency is to replace the radial plot analysis with the level of confidence analysis for both analyte identification and concentration determination to be performed simultaneously. In this case, confidence levels should be calculated in a sixdimensional space by including the steady-state frequency shifts and time responses of three sensor coatings. More data points can also be collected, within the same range of $0.5 \mathrm{mg} / \mathrm{L}$ to $3.0 \mathrm{mg} / \mathrm{L}$, to increase the database needed for a more accurate analyte 
identification.

In addition to improving the single-component identification process, an investigation can be performed on using the same pattern recognition technique to identify binary mixtures [44]. A new "fingerprint" can be developed using the same radial plot analysis. After identifying the analyte in the mixture, the problem of the unknown ratio or concentration of chemical components needs to be solved. A database can be built, containing known ratios and chemical components of the binary mixtures, for the use of the pattern recognition algorithm. Once the unknown binary mixture is tested, the levels of confidence $\mathrm{C}_{1}$ and $\mathrm{C}_{2}$ corresponding to concentrations $\mathrm{c}_{1}$ and $\mathrm{c}_{2}$ can be obtained [44]. Using methods found in Ref. 44, the ratio and concentration of the binary mixture can be calculated. 


\section{REFERENCES}

[1] P. Quinn, "Organophosphates," Illinois Department of Public Health, [Online]. Available: http://www.idph.state.il.us/Bioterrorism/factsheets/organophosphate.htm. [Accessed 26 July 2012].

[2] "Nerve Agents," [Online]. Available: http://www.gulflink.osd.mil/library/randrep/mr1018.5.ch5.pdf. [Accessed 26 July 2012].

[3] "Nerve Agent and Organophosphate Pesticide Poisoning," Centers for Disease Control and Prevention, 2006. [Online]. Available: http://www.bt.cdc.gov/agent/nerve/tsd.asp. [Accessed 26 July 2012].

[4] "Organophosphates," WebMD, 1194-2012. [Online]. Available: http://emedicine.medscape.com/article/1175139-overview. [Accessed 26 July 2012].

[5] "Pesticides: Regulating Pesticides," Environmental Protection Agency , 25 September 2012. [Online]. Available: http://www.epa.gov/pesticides/regulating/index.htm. [Accessed 26 July 2012].

[6] M. Tankiewicz, J. Fenik and M. Biziuk, "Determination of organophosphorus and organonitrogen pesticides in water samples," Trends in Analytical Chemistry, vol. 29, no. 9, pp. 1050-1063, 2010.

[7] J. Fraden, Handbook of Modern Sensors: Phsyics, Design and Applications, New York: Springer-Verlag Inc, 2004.

[8] J. R. Stetter, W. R. Penrose and S. Yao, "Sensors, Chemical Sensors, Electrochemical Sensors, and ECS," Journal of The Electrochemical Society, vol. 150, no. 2, pp. S11-S16, 2003.

[9] X. Zhu, "Nano Electrochemical Sensor and its Measurement Electronics with a Dynamic Transduction Mechanism," University of Cincinnati / OhioLINK, Cincinnati, 2005.

[10] B. Drafts, "Acoustic Wave Technology Sensors," IEEE Transactions on Microwave Theory and Techniques, vol. 49, no. 4, pp. 795-802, 2001.

[11] W. H. King, "Piezoelectric sorption detector," Anal. Chem., vol. 36, pp. 1735-1739, 
1964.

[12] R. White and F. Voltmer, "Direct piezoelectric coupling to surfaceelastic waves," Appl. Phys. Lett., vol. 7, pp. 314-316, 1965.

[13] N. R. Smith, "Increasing the Sensitivity of Surface Acoustic Wave (SAW) Chemical Sensors and other Chemical Sensing Investigations," Air Force Institute of Technology, Wright-Patterson Air Force Base, Ohio, 1959.

[14] L. Rayleigh, "On Waves Propagated along the Plane Surface of an Elastic Solid," Proceedings of the London Mathematical Society, vol. 17, pp. 4-11, 1885.

[15] E. Gizeli, "Design considerations for the acoustic waveguide biosensor," Smart Mater. Struct., vol. 6, pp. 700-706, 1997.

[16] E. T. Zellets, S. A. Batterman and M. Ha, "Optimal Coating Selection for the Analysis of Organic Vapor Mixtures with Polymer-Coated Surface Acoustic Wave Sensor Arrays," Anal. Chem., vol. 67, pp. 1092-1106, 1995.

[17] F. Bender, R. W. Cernosek and F. Josse, "Love-wave biosensors using cross-linked polymer waveguides on LiTaO3 substrates," Electronics Letters, vol. 19, no. 16721673, p. 36, 2000.

[18] K. Zinszer, R. Zhou, F. Josse, F. Bender and R. W. Cernosek, "Optimization of SHSAW liquid sensor platforms for (bio-)chemical detection using polymer," Acoustic Wave-Based Sensors Symp, vol. 198th ECS Mtg., p. 1070, 2000.

[19] F. Josse, F. Bender and R. W. Cernosek, "Guided shear horizontal surface acoustic wave sensors for chemical and biochemical detection in liquids," Anal. Chem., vol. 73, pp. 5937-5944, 2001.

[20] Y. Gulyaev, "Review of Shear Surface Acoustic Waves in Solids," IEEE Trans. UFFC, vol. 45, no. 4, pp. 935-938, 1998.

[21] D. S. Ballantine, R. M. White, S. J. Martin, H. Wohltjen and A. J. Ricco, Acoustic Wave Sensors: Theory, Design, and Physico-Chemical Applications, San Diego: Academic Press, 1997.

[22] S. J. Martin, G. C. Frye and S. D. Senturia, "Dynamics and Response of PolymerCoated Surface Acoustic Wave Devices: Effect of Viscoelastic Properties and Film Response," Anal. Chem., vol. 66, pp. 2201-2219, 1994. 
[23] G. L. Harding, J. Du, P. R. Dencher, D. Barnett and E. Howe, "Love wave acoustic immunosensor operating in liquid," Sensors and Actuators, vol. A61, pp. 279-286, 1997.

[24] A. K. Mensah-Brown, "Detection of Organophosphates in the Liquid-Phase Using Guided SH-SAW Sensors [Thesis]," Marquette University, Milwaukee, 2007.

[25] K. Bodenhofer, A. Hierlemann, G. Noetzel, U. Weimar and W. Gopel, "Performances of Mass-Sensitive Devices for Gas Sensing: Thickness Shear Mode and Surface Acoustic Wave Transducers," Anal. Chem., vol. 68, pp. 2210-2218, 1996.

[26] Z. Li, "Guided Shear-Horizontal Surface Acoustic Wave (SH-SAW) Chemical Sensors for Detection of Organic Contaminants in Aqueous Environments [Dissertation]," Marquette University, Milwaukee, 2005.

[27] A. Mensah-Brown, "Detection of Organophosphates in the Liquid-Phase Using Guided SH-SAW Sensors [Thesis]," Marquette University, Milwaukee, 2007.

[28] J. W. Grate and S. J. Patrash, "Method for Estimating Polymer-Coated Acoustic Wave Vapor Sensor Responses," Anal. Chem., vol. 67, pp. 2162-2169, 1995.

[29] A. K. Mensah-Brown, M. J. Wenzel, F. J. Josse and E. E. Yaz, "Near Real-Time Monitoring of Organophosphate Pesticides in the Aqueous-Phase Using SH-SAW Sensors Including Estimation-Based Signal Analysis," IEEE Sensors Journal, vol. 9, no. 12, pp. 1817-1824, 2009.

[30] J. W. Grate and E. T. Zellers, "The Fractional Free Volume of the Sorbed Vapor in Modeling the Viscoelastic Contribution to Polymer-Coated Surface Acoustic Wave Vapor Sensor Responses," Anal. Chem., vol. 72, pp. 2861-2868, 2000.

[31] A. Hierlemann, E. T. Zellers and A. J. Ricco, "Use of Linear Solvation Energy Relationships for Modeling Responses from Polymer-Coated Acoustic-Wave Vapor Sensors," Anal. Chem., vol. 73, pp. 3458-3466, 2001.

[32] F. Bender, R. W. Cernosek and F. Josse, "Love-wave biosensors using cross-linked polymer waveguides on LiTaO3 substrates," Electronics Letters, vol. 36, no. 19, pp. 1672-1673, 200.

[33] F. Josse and R. Cernosek.

[34] M. J. Levine, Pesticides: A Toxic Time Bomb in Our Midst, Westport: Praeger 
Publishers, 2007.

[35] "Material Safety Data Sheet: Parathion.," Sigma Aldrich, 2012. [Online]. Available: http://www.sigmaaldrich.com/catalog/search/ProductDetail/RIEDEL/45607. [Accessed 15 May 2012].

[36] D. L. Lide, Aqueous Solubility and Henry's Law Constants of Organic Compounds. Hanbook of Chemisty and Physics, 82nd ed, Raton: CRC Press, 2004.

[37] "Material Safety Data Sheet: Parathion-methyl," Sigma Aldrich, 2012. [Online]. Available:

http://www.sigmaaldrich.com/catalog/product/fluka/36187?lang=en\&region=US. [Accessed 15 May 2012].

[38] "Material Safety Data Sheet: Paraoxon," Sigma-Aldrich, 9 May 2012. [Online]. Available: http://www.sigmaaldrich.com/catalog/product/supelco/ps610?lang=en\&region=US. [Accessed 15 May 2012].

[39] A. K. Mensah-Brown, "Analysis of the Detection of Organophosphate Pesticides in Aqueous Solutions Using Polymer-Coated SH-SAW Devices [Dissertation]," Marquette University, Milwaukee, 2010.

[40] J. W. Grate and D. A. Nelson, "A. Polymers for Chemical Sensors Using Hydrosilylation Chemistry," Pacific Northwest National Laboratory, Richland, 2001.

[41] "Spin Coating Theory," 2012. [Online]. Available: http://www.cise.columbia.edu/clean/process/spintheory.pdf. [Accessed 15 May 2012].

[42] "Ellipsometers," Gaertner Scientific Corporation , 2008-2012. [Online]. Available: http://www.gaertnerscientific.com/ellipsometers/main.htm. [Accessed 15 May 2012].

[43] R. S. Fisher, "Groundwater Quality in Kentucky: pH," University of Kentucky, Lexington, 2002.

[44] R. Dahint, Z. A. Shana, F. Josse, S. A. Riedel and M. Grunze, "Identification of Metal Ion Solutions Using Acoustic Plate Mode Devices and Pattern Recognition," in IEEE Transactions on Ultrasonics, Ferroelectrics, and Frequency Control, 1993.

[45] F. Bender, A. Skrypnik, A. Voigt, J. Marcoll and M. Rapp, "Selective Detection of 
HFC and HCFC Refrigerants Using a Surface Acoustic Wave Sensor System," Anal. Chem., vol. 75, pp. 5262-5266, 2003.

[46] U.S. Environmental Protection Agency (EPA), "List of Contaminants \& their MCLs," 05 June 2012. [Online]. Available: http://water.epa.gov/drink/contaminants/index.cfm\#List. [Accessed 19 September 2012].

[47] H. R. McLean, S. Futagaki and J. T. Leffingwell, "Loss of Paraoxon in Aqueous Acetonitrile Extractions," BULLETIN OF ENVIRONMENTAL CONTAMINATION AND TOXICOLOGY, vol. 18, no. 2, pp. 247-250, 1977.

[48] Y. Jones, Z. Li, F. Josse and J. Hossenlopp, "Quantitative Characterization of the Partitioning of Aqueous Analytes into a Polymer Coating," in Proceedings of IEEE Sensors, 2003.

[49] J. W. Grate, M. Klusty, R. A. McGill, M. H. Abraham, G. Whiting and J. AndonianHaftavan, "The Predominant Role of Swelling-Induced Modulus Changes of the Sorbent Phase in Determining the Responses of Polymer-Coated Surface Acoustic Wave Vapor Sensors," Anal. Chem., vol. 64, pp. 610-624, 1992. 


\section{APPENDIX}

\begin{tabular}{|c|c|c|c|c|c|c|}
\hline & \multicolumn{6}{|c|}{ Sensitivity $(\mathrm{kHz} / \mathrm{mg} / \mathrm{L}$} \\
\hline polymer & $\begin{array}{c}\text { BPA- } \\
\text { HMTS } \\
\text { HPA- }\end{array}$ & $\begin{array}{c}\text { PECH } \\
\text { PDMS } \\
0.25 \mu \mathrm{m}\end{array}$ & $\begin{array}{l}\text { BPA- } \\
0.25 \mu \mathrm{m}\end{array}$ & $\begin{array}{c}\text { BPA- } \\
\text { HMTS }\end{array}$ & $\begin{array}{c}\text { PECH } \\
\text { PDMS }\end{array}$ & $0.5 \mu \mathrm{m}$ \\
$0.5 \mu \mathrm{m}$ & $0.5 \mu \mathrm{m}$ & \\
\hline parathion & 2.449 & 1.073 & 1.491 & 20.791 & 9.002 & 9.093 \\
\hline parathion-methyl & 0.572 & 0.196 & 0.512 & 4.246 & 0.725 & 2.278 \\
\hline paraoxon & 0.450 & 0.049 & 0.026 & 4.413 & 0.489 & 0.175 \\
\hline
\end{tabular}

Table A.1: The sensitivities for $0.25 \mu \mathrm{m}$ and $0.5 \mu \mathrm{m}$ thick BPA-HMTS, BPA-PDMS, and PECH when exposed to parathion, parathion-methyl, and paraoxon.

\begin{tabular}{|c|c|c|c|c|c|c|}
\hline & \multicolumn{7}{|c|}{ Sensitivity $(\mathrm{kHz} / \mathrm{mg} / \mathrm{L})$} \\
\hline polymer & BPA- & BPA- & PECH & BPA- & BPA- & PECH \\
& PMTS & PDMS & $0.25 \mu \mathrm{m}$ & HMTS & PDMS & $0.5 \mu \mathrm{m}$ \\
& $0.25 \mu \mathrm{m}$ & $0.25 \mu \mathrm{m}$ & & $0.5 \mu \mathrm{m}$ & $0.5 \mu \mathrm{m}$ & \\
\hline parathion & 31.598 & 13.844 & 19.244 & 268.203 & 116.130 & 117.300 \\
\hline parathion-methyl & 21.761 & 7.476 & 19.483 & 161.374 & 27.571 & 86.567 \\
\hline paraoxon & 1080.144 & 118.891 & 63.988 & 10593.360 & 1174.320 & 420.048 \\
\hline
\end{tabular}

Table A.2: The sensitivities for $0.25 \mu \mathrm{m}$ and $0.5 \mu \mathrm{m}$ thick BPA-HMTS, BPA-PDMS, and PECH when exposed to parathion, parathion-methyl, and paraoxon, normalized to solubility. 


\begin{tabular}{|c|c|c|c|c|c|c|}
\hline & \multicolumn{3}{|c|}{ Frequency Shift $(\mathrm{kHz})$} & \multicolumn{3}{|c|}{ Time Response (minutes) } \\
\hline $\begin{array}{c}\text { analyte } \\
\text { concentration }\end{array}$ & $\begin{array}{l}\mathrm{PECH} \\
0.5 \mu \mathrm{m}\end{array}$ & $\begin{array}{l}\text { BPA- } \\
\text { HMTS } \\
0.5 \mu \mathrm{m}\end{array}$ & $\begin{array}{l}\text { BPA- } \\
\text { PDMS } \\
0.5 \mu \mathrm{m}\end{array}$ & $\begin{array}{l}\text { PECH } \\
0.5 \mu \mathrm{m}\end{array}$ & $\begin{array}{c}\text { BPA- } \\
\text { HMTS } \\
0.5 \mu \mathrm{m}\end{array}$ & $\begin{array}{c}\text { BPA- } \\
\text { PDMS } \\
0.5 \mu \mathrm{m}\end{array}$ \\
\hline parathion $(0.5 \mathrm{mg} / \mathrm{L})$ & -1.585 & -5.500 & 2.890 & 58.218 & 279.427 & 49.148 \\
\hline parathion $(1.0 \mathrm{mg} / \mathrm{L})$ & -7.071 & -35.361 & 4.652 & 282.972 & 719.305 & 82.769 \\
\hline parathion $(1.5 \mathrm{mg} / \mathrm{L})$ & -11.254 & -36.349 & 7.7474 & 261.583 & 585.360 & 87.869 \\
\hline parathion $(2.0 \mathrm{mg} / \mathrm{L})$ & -14.300 & -40.503 & 10.578 & 284.800 & 341.655 & 66.429 \\
\hline parathion $(2.5 \mathrm{mg} / \mathrm{L})$ & -23.951 & -52.894 & 25.286 & 310.605 & 580.973 & 108.003 \\
\hline parathion $(3.0 \mathrm{mg} / \mathrm{L})$ & -31.214 & -58.974 & 34.238 & 201.562 & 495.029 & 105.657 \\
\hline
\end{tabular}

Table A.3: The frequency shifts and time responses of BPA-HMTS, BPA-PDMS, and PECH at a thickness of $0.5 \mu \mathrm{m}$ when exposed to $0.5 \mathrm{mg} / \mathrm{L}-3.0 \mathrm{mg} / \mathrm{L}$ of parathion.

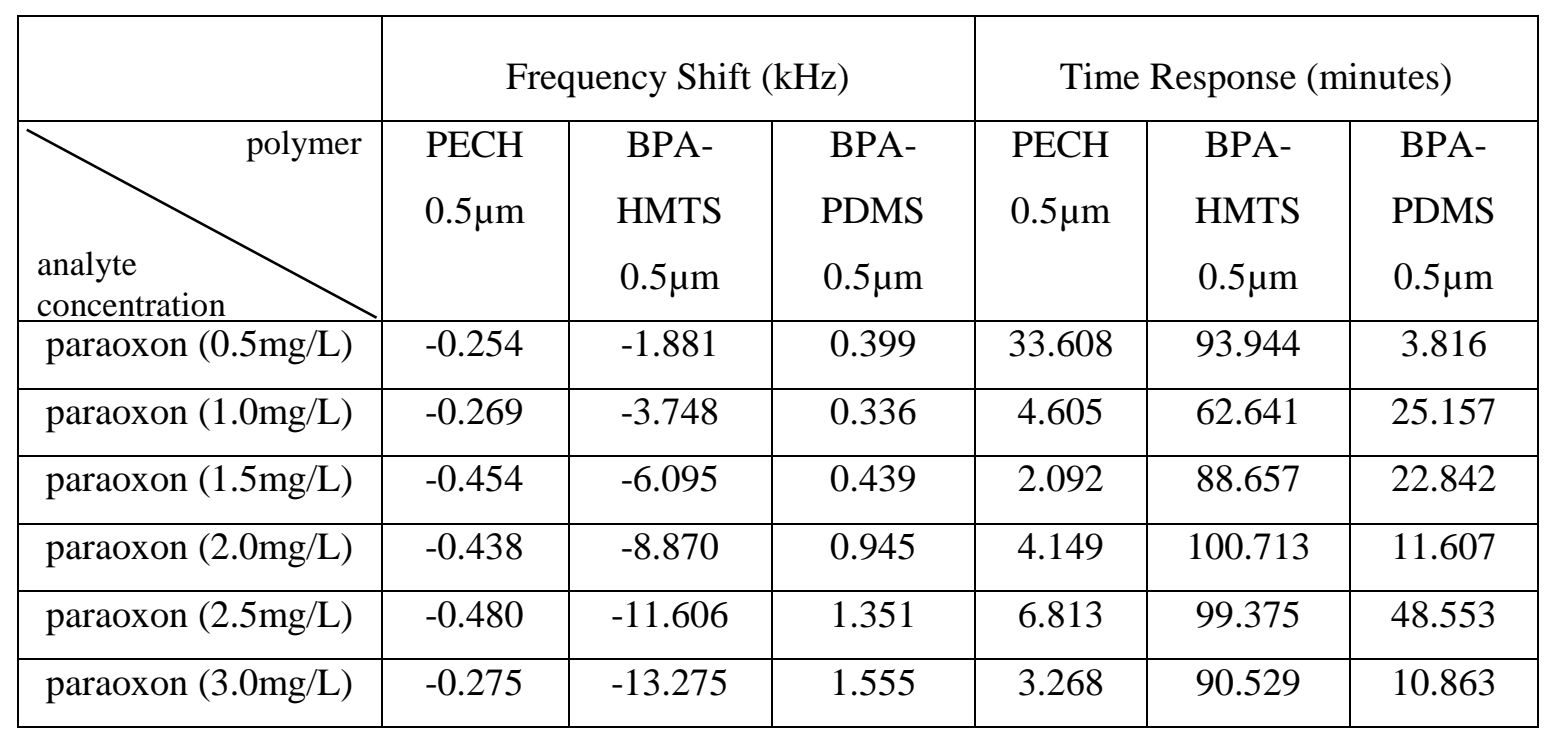

Table A.4: The frequency shifts and time responses of BPA-HMTS, BPA-PDMS, and PECH at a thickness of $0.5 \mu \mathrm{m}$ when exposed to $0.5 \mathrm{mg} / \mathrm{L}-3.0 \mathrm{mg} / \mathrm{L}$ of paraoxon. 


\begin{tabular}{|c|c|c|c|c|c|c|}
\hline & \multicolumn{3}{|c|}{ Frequency Shift (kHz) } & \multicolumn{3}{c|}{ Time Response (minutes) } \\
\hline \multirow{2}{*}{$\begin{array}{c}\text { polymer } \\
\text { analyte }\end{array}$} & $\begin{array}{c}\text { PECH } \\
0.5 \mu \mathrm{m}\end{array}$ & $\begin{array}{c}\text { BPA- } \\
\text { HMTS } \\
0.5 \mu \mathrm{m}\end{array}$ & $\begin{array}{c}\text { BPA- } \\
\text { PDMS } \\
0.5 \mu \mathrm{m}\end{array}$ & $\begin{array}{c}\text { PECH } \\
0.5 \mu \mathrm{m}\end{array}$ & $\begin{array}{c}\text { BPA- } \\
\text { HMTS } \\
0.5 \mu \mathrm{m}\end{array}$ & $\begin{array}{c}\text { BPA- } \\
\text { PDMS } \\
0.5 \mu \mathrm{m}\end{array}$ \\
\hline $\begin{array}{c}\text { parathion-methyl } \\
(0.5 \mathrm{mg} / \mathrm{L})\end{array}$ & -0.680 & -2.134 & 0.422 & 43.164 & 100.389 & 12.174 \\
\hline $\begin{array}{c}\text { parathion-methyl } \\
(1.0 \mathrm{mg} / \mathrm{L})\end{array}$ & -2.008 & -3.963 & 0.682 & 53.038 & 94.439 & 20.631 \\
\hline $\begin{array}{c}\text { parathion-methyl } \\
(1.5 \mathrm{mg} / \mathrm{L})\end{array}$ & -3.581 & -5.927 & 1.084 & 56.803 & 100.957 & 23.458 \\
\hline $\begin{array}{c}\text { parathion-methyl } \\
(2.0 \mathrm{mg} / \mathrm{L})\end{array}$ & -5.009 & -7.857 & 1.489 & 56.885 & 96.409 & 18.812 \\
\hline $\begin{array}{c}\text { parathion-methyl } \\
(2.5 \mathrm{mg} / \mathrm{L})\end{array}$ & -5.816 & -10.583 & 1.706 & 46.067 & 103.779 & 11.799 \\
\hline $\begin{array}{c}\text { parathion-methyl } \\
(3.0 \mathrm{mg} / \mathrm{L})\end{array}$ & -6.515 & -13.505 & 2.246 & 47.835 & 108.424 & 19.042 \\
\hline
\end{tabular}

Table A.5: The frequency shifts and time responses of BPA-HMTS, BPA-PDMS, and PECH at a thickness of $0.5 \mu \mathrm{m}$ when exposed to $0.5 \mathrm{mg} / \mathrm{L}-3.0 \mathrm{mg} / \mathrm{L}$ of parathion-methyl. 